Supplement of Earth Syst. Dynam., 12, 1543-1569, 2021

https://doi.org/10.5194/esd-12-1543-2021-supplement

(C) Author(s) 2021. CC BY 4.0 License.

(c) (1)

Earth System

Dynamics

EGU

Supplement of

\title{
Balanced estimate and uncertainty assessment of European climate change using the large EURO-CORDEX regional climate model ensemble
}

\section{Guillaume Evin et al.}

Correspondence to: Guillaume Evin (guillaume.evin@inrae.fr)

The copyright of individual parts of the supplement might differ from the article licence. 


\section{Characteristics of the scenarios}

Table S1. Characteristics of the scenarios of the EURO-CORDEX climate projection ensemble.

\begin{tabular}{|c|c|c|c|c|}
\hline $\mathrm{RCP}$ & GCM & Member & RCM & Version \\
\hline & CanESM2 & rlilp1 & CCLM4-8-17 & v1 \\
\hline rep85 & CanESM2 & rlilp1 & REMO & $\mathrm{v} 1$ \\
\hline rcp26 & CNRM-CM5 & rlilp1 & ALADIN53 & $\mathrm{v} 1$ \\
\hline rcp45 & CNRM-CM5 & rlilpl & ALADIN53 & $\mathrm{v} 1$ \\
\hline rep85 & CNRM-CM5 & rlilp1 & ALADIN53 & $\mathrm{v} 1$ \\
\hline rcp26 & CNRM-CM5 & rlilp1 & ALADIN63 & v2 \\
\hline rcp45 & CNRM-CM5 & rlilpl & ALADIN63 & v2 \\
\hline rep85 & CNRM-CM5 & rlilpl & ALADIN63 & v2 \\
\hline rep26 & CNRM-CM5 & rlilp1 & ALARO-0 & $\mathrm{v} 1$ \\
\hline rep45 & CNRM-CM5 & rlilpl & ALARO-0 & $\mathrm{v} 1$ \\
\hline rep85 & CNRM-CM5 & rlilpl & ALARO-0 & $\mathrm{v} 1$ \\
\hline rep45 & CNRM-CM5 & rlilp1 & CCLM4-8-17 & v1 \\
\hline rep85 & CNRM-CM5 & rlilp1 & CCLM4-8-17 & v1 \\
\hline rep85 & CNRM-CM5 & rlilp1 & HIRHAM5 & v2 \\
\hline rep26 & CNRM-CM5 & rlilp1 & RACMO22E & v2 \\
\hline rcp45 & CNRM-CM5 & rlilp1 & RACMO22E & $\mathrm{v} 2$ \\
\hline rep85 & CNRM-CM5 & rlilp1 & RACMO22E & v2 \\
\hline rcp45 & CNRM-CM5 & rlilp1 & RCA4 & $\mathrm{v} 1$ \\
\hline rcp85 & CNRM-CM5 & rlilp1 & RCA4 & $\mathrm{v} 1$ \\
\hline rep85 & CNRM-CM5 & rlilpl & REMO & $\mathrm{v} 1$ \\
\hline rep85 & CNRM-CM5 & rlilp1 & WRF381P & v2 \\
\hline rcp26 & EC-EARTH & r12ilp1 & CCLM4-8-17 & $\mathrm{v} 1$ \\
\hline rcp45 & EC-EARTH & r12ilp1 & CCLM4-8-17 & $\mathrm{v} 1$ \\
\hline rep85 & EC-EARTH & r12ilp1 & CCLM4-8-17 & v1 \\
\hline rcp85 & EC-EARTH & r12ilpl & HIRHAM5 & v1 \\
\hline rep26 & EC-EARTH & r12ilp1 & RACMO22E & v1 \\
\hline rcp45 & EC-EARTH & r12ilp1 & RACMO22E & $\mathrm{v} 1$ \\
\hline rep85 & EC-EARTH & r12ilp1 & RACMO22E & v1 \\
\hline rep26 & EC-EARTH & r12ilp1 & RCA4 & $\mathrm{v} 1$ \\
\hline rep45 & EC-EARTH & r12ilp1 & RCA4 & $\mathrm{v} 1$ \\
\hline rep85 & EC-EARTH & r12ilp1 & RCA4 & $\mathrm{v} 1$ \\
\hline rep26 & EC-EARTH & r12ilp1 & REMO & $\mathrm{v} 1$ \\
\hline rep85 & EC-EARTH & r12ilp1 & REMO & $\mathrm{v} 1$ \\
\hline rep85 & EC-EARTH & r12ilpl & WRF361H & v1 \\
\hline rcp26 & GFDL-ESM2G & rlilpl & REMO & $\mathrm{v} 1$ \\
\hline rep85 & HadGEM2-ES & rlilp1 & ALADIN63 & $\mathrm{v} 1$ \\
\hline rep45 & HadGEM2-ES & rlilp1 & CCLM4-8-17 & v1 \\
\hline rcp85 & HadGEM2-ES & rlilp1 & CCLM4-8-17 & $\mathrm{v} 1$ \\
\hline rcp85 & HadGEM2-ES & rlilp1 & HadREM3-GA7 & $\mathrm{v} 1$ \\
\hline rep45 & HadGEM2-ES & rlilp1 & HIRHAM5 & v2 \\
\hline rcp85 & HadGEM2-ES & rlilp1 & HIRHAM5 & v2 \\
\hline rcp26 & HadGEM2-ES & rlilp1 & RACMO22E & v2 \\
\hline rep45 & HadGEM2-ES & rlilp1 & RACMO22E & v2 \\
\hline rep85 & HadGEM2-ES & rlilp1 & RACMO22E & v2 \\
\hline rcp26 & HadGEM2-ES & rlilpl & RCA4 & $\mathrm{v} 1$ \\
\hline rcp45 & HadGEM2-ES & rlilp1 & RCA4 & $\mathrm{v} 1$ \\
\hline rep85 & HadGEM2-ES & rlilp1 & RCA4 & $\mathrm{v} 1$ \\
\hline rep26 & HadGEM2-ES & rlilp1 & RegCM4-6 & v1 \\
\hline rep85 & HadGEM2-ES & rlilp1 & RegCM4-6 & $\mathrm{v} 1$ \\
\hline rep26 & HadGEM2-ES & rlilpl & REMO & $\mathrm{v} 1$ \\
\hline rep85 & HadGEM2-ES & rlilp1 & REMO & $\mathrm{v} 1$ \\
\hline rep85 & HadGEM2-ES & rlilp1 & WRF361H & $\mathrm{v} 1$ \\
\hline rep85 & HadGEM2-ES & rlilpl & WRF381P & $\mathrm{v} 1$ \\
\hline rcp85 & IPSL-CM5A-MR & rlilp1 & RACMO22E & $\mathrm{v} 1$ \\
\hline rcp45 & IPSL-CM5A-MR & rlilp1 & RCA4 & $\mathrm{v} 1$ \\
\hline rep85 & IPSL-CM5A-MR & rlilp1 & RCA4 & $\mathrm{v} 1$ \\
\hline rcp45 & IPSL-CM5A-MR & rlilp1 & WRF381P & $\mathrm{v} 1$ \\
\hline rep85 & IPSL-CM5A-MR & rlilp1 & WRF381P & $\mathrm{v} 1$ \\
\hline rсp26 & MIROC5 & rlilp1 & CCLM4-8-17 & $\mathrm{v} 1$ \\
\hline rcp85 & MIROC5 & rlilp1 & CCLM4-8-17 & $\mathrm{v} 1$ \\
\hline rep26 & MIROC5 & rlilp1 & REMO & v1 \\
\hline rep85 & MIROC5 & rlilp1 & REMO & $\mathrm{v} 1$ \\
\hline rep85 & MIROC5 & rlilp1 & WRF361H & $\mathrm{v} 1$ \\
\hline rep26 & MPI-ESM-LR & rlilp1 & CCLM4-8-17 & $\mathrm{v} 1$ \\
\hline rep45 & MPI-ESM-LR & rlilp1 & CCLM4-8-17 & $\mathrm{v} 1$ \\
\hline rep85 & MPI-ESM-LR & rlilpl & CCLM4-8-17 & $\mathrm{v} 1$ \\
\hline rep85 & MPI-ESM-LR & rlilp1 & COSMO-crCLIM & $\mathrm{v} 1$ \\
\hline rcp85 & MPI-ESM-LR & rlilp1 & HIRHAM5 & $\mathrm{v} 1$ \\
\hline rep85 & MPI-ESM-LR & rlilpl & RACMO22E & $\mathrm{v} 1$ \\
\hline rcp26 & MPI-ESM-LR & rlilp1 & RCA4 & vla \\
\hline rep45 & MPI-ESM-LR & rlilp1 & RCA4 & vla \\
\hline rep85 & MPI-ESM-LR & rlilp1 & RCA4 & vla \\
\hline rcp85 & MPI-ESM-LR & rlilp1 & RegCM4-6 & $\mathrm{v} 1$ \\
\hline rep26 & MPI-ESM-LR & rlilpl & REMO & $\mathrm{v} 1$ \\
\hline rep45 & MPI-ESM-LR & rlilp1 & REMO & v1 \\
\hline rep85 & MPI-ESM-LR & rlilp1 & REMO & v1 \\
\hline rcp26 & MPI-ESM-LR & rlilp1 & WRF361H & $\mathrm{v} 1$ \\
\hline rep85 & MPI-ESM-LR & rlilp1 & WRF361H & $\mathrm{v} 1$ \\
\hline rep85 & NorESM1-M & rlilp1 & COSMO-crCLIM & $\mathrm{v} 1$ \\
\hline rcp45 & NorESM1-M & rlilpl & HIRHAM5 & \\
\hline rep85 & NorESM1-M & rlilpl & HIRHAM5 & v2 \\
\hline rep85 & NorESM1-M & rlilpl & RACMO22E & $\mathrm{v} 1$ \\
\hline rep26 & NorESM1-M & rlilp1 & RCA4 & $\mathrm{v} 1$ \\
\hline & NorESM1- & rlilp1 & $\mathrm{RC}$ & $\mathrm{v} 1$ \\
\hline rep26 & NorESM1-M & rlilp1 & REMO & $\mathrm{v} 1$ \\
\hline rep 85 & NorESM1-M & rlilp1 & REMO & v1 \\
\hline rcp85 & NorESM1-M & rlilpl & WRF381P & $\mathrm{v} 1$ \\
\hline
\end{tabular}




\section{Detailed description of the QUALYPSO method}

In the QUALYPSO approach, each climate projection is decomposed into a smooth signal (the climate response) and a noise (internal variability). This so-called "time-series" approach (Hawkins and Sutton, 2009; Hingray and Saïd, 2014) first consists in extracting the climate response of each climate experiment from the raw projection. In QUALYPSO, cubic smoothing splines are used to extract the climate response for each simulation chain. Deviations from these climate responses are considered to be the result of internal variability. For each chain, the climate change response function is obtained from the differences between the climate responses obtained for different future periods and a reference period. The ensemble of climate change responses obtained for the different chains is used to assess the grand ensemble mean of projected changes and associated uncertainties. For further details on the method, we refer the reader to Evin et al. (2019). The four sequential steps can be summarized as follows:

1. The first step is related to the extraction of the climate responses and the estimation of the internal variability.

2. The second step is related to the computation of the climate change responses (i.e. anomalies).

3. The third step is an ANalysis Of VAriance (ANOVA): the ensemble of climate change responses is decomposed using an additive model composed of a grand mean and main effects.

4. The fourth step further decomposes the remaining variability into interaction effects and residual variability.

In this study, we consider an ensemble of simulation chains where each chain corresponds to a given GCM/RCM combination for a given emission scenario (RCP). For a $\operatorname{RCM} i, \operatorname{GCM} j$ and scenario $k, Y_{i, j, k}(t)$ denotes the raw climate projections of the climate variable (temperature, precipitation) for a given time $t$. The EURO-CORDEX climate projection ensemble described in Table $\mathrm{S} 1$ is made of $n_{o}=87$ experiments corresponding to different simulation chains. The number of available experiments is smaller than the total number of possible climate "experiments" (denoted by $n$ ), that would correspond to all possible combinations of the different scenarios/models considered here. In this study, there are $n=351$ possible combinations ( $I=13$ different RCMs times $J=9$ different GCMs times $K=3$ different RCPs), and $n_{m}=264$ combinations are thus missing.

\subsection{Step 1: Extraction of the climate responses}

For each simulation chain, the raw climate projections $Y_{i, j, k}(t)$ can be expressed as:

$Y_{i, j, k}(t)=\phi_{i, j, k}(t)+\eta_{i, j, k}(t)$

where $\phi_{i, j, k}(t)$ is the climate response for this simulation chain and $\eta_{i, j, k}(t)$ is the deviation from the climate response, as a result of internal variability. In this paper, the climate response $\phi_{i, j, k}(t)$ is obtained by fitting a cubic spline to the time series $Y_{i, j, k}(t)$ (Evin et al., 2019). 


\subsection{Step 2: Computation of the change variables}

As in most climate impact studies, uncertainty sources are quantified from change variables, obtained as differences between a future and a reference period. The change variable can be defined in terms of absolute changes $Y_{i, j, k}^{*}(t)=Y_{i, j, k}(t)-\phi_{i, j, k}(c)$ (e.g. for temperature), or in terms of relative changes $Y_{i, j, k}^{*}(t)=Y_{i, j, k}(t) / \phi_{i, j, k}(c)-1$ (e.g. for precipitation), where $\phi_{i, j, k}(c)$ is the value of the climate response for the reference period $c=1981-2010$. In both cases, the change variable $Y_{i, j, k}^{*}(t)$ can be split up into $Y_{i, j, k}^{*}(t)=\phi_{i, j, k}^{*}(t)+\eta_{i, j, k}^{*}(t)$ where $\phi_{i, j, k}^{*}(t)$ is the climate change response for this simulation chain and time $t$, and $\eta_{i, j, k}^{*}(t)$ is the deviation from the climate change response, as a result of internal variability.

\subsection{Step 3: Decomposition of the climate change responses}

This study aims at applying an ANOVA decomposition to the climate change response $\phi_{i, j, k}^{*}(t)$ in order to obtain the part of its variability corresponding to the different components (RCMs, GCMs, etc.). In QUALYPSO, the climate change response is expressed as the sum of the grand mean, the main effects, and a residual term, i.e.:

$\phi_{i, j, k}^{*}(t)=\mu(t)+\alpha_{i}(t)+\beta_{j}(t)+\gamma_{k}(t)+\xi_{i, j, k}(t)$,

where

- $\mu(t)$ is the ensemble mean climate change response, shared by all simulation chains,

- $\alpha_{i}(t), \beta_{j}(t)$ and $\gamma_{k}(t)$ are the main effects corresponding to the type of RCM, GCM and emission scenario, respectively,

- $\xi_{i, j, k}(t)=\phi_{i, j, k}^{*}(t)-\mu(t)-\alpha_{i}(t)-\beta_{j}(t)-\gamma_{k}(t)$ is a residual term which represents the part of the climate change response that cannot be explained by the sum of the ensemble mean and the main effects.

The QUALYPSO approach (Evin et al., 2019) consists in estimating the different terms of Eq. S2 (grand mean $\mu(t)$ and the main effects $\alpha_{i}(t), \beta_{j}(t)$ and $\left.\gamma_{k}(t)\right)$ using a Bayesian framework and data augmentation. Missing climate projections are part of the inference and the posterior distributions of all unknown quantities (grand mean, main effects and missing climate projections) are sampled sequentially using the Gibbs algorithm. In this study, the mean of the posterior distributions are taken as estimates and are denoted by $\hat{\mu}(t)$ for the grand mean, and $\hat{\alpha}_{i}(t), \hat{\beta}_{j}(t)$ and $\hat{\gamma}_{k}(t)$ for the main effects. The mean expected change $B M_{k}$ for each scenario $k$ and time $t$ is the sum of the ensemble mean climate change response $\hat{\mu}(t)$ and the scenario effect:

$B M_{k}=\hat{\mu}(t)+\hat{\gamma}_{k}(t)$.

\subsection{Step 4: Residual variability and interaction effects}

The residual term $\xi_{i, j, k}(t)$ in Eq. (S2) characterizes the part of the climate change response which is not the result of a systematic effect of RCM and GCM models, or of the emission scenario (so-called main effects). This variability can be due to interactions between the emission scenarios and the climate models (RCP/GCM, RCP/RCM) or between the climate models 
(GCM/RCM). For complete ensembles, these interactions can be estimated using all simulation chains corresponding to a specific combination (see, e.g. Déqué et al., 2007; Yip et al., 2011). The last remaining variability is related to interactions RCM/GCM/RCP, which can be estimated using different replicates of the simulation chains (Yip et al., 2011) or by making additional assumptions, e.g. by considering residual terms corresponding to successive years as replicates (Christensen and Kjellström, 2020). Northrop and Chandler (2014) note that direct estimates of these interactions are often biased. In our case, only one member of each simulation chain is available, and the QUALYPSO approach cannot be considered to estimate the very large number of possible interaction effects. For example, the data augmentation approach would consist in estimating $I \times J=117$ additional terms for GCM/RCM interactions, which is not feasible considering that only $n_{o}=87$ simulation chains are available.

In this study, we assess the variability related to the three types of interactions (RCM/GCM, RCM/RCP, GCM/RCP) by applying an additional decomposition of residual terms $\xi_{i, j, k}(t)$. The remaining variability (interactions RCM/GCM/RCP) is considered as the residual variability. Heuristic estimates of interaction effects and residual variability are obtained as follows:

- For all available simulation chains, we first obtain residual terms as:

$$
\hat{\xi}_{i, j, k}(t)=\phi_{i, j, k}^{*}(t)-\hat{\mu}(t)-\hat{\alpha}_{i}(t)-\hat{\beta}_{j}(t)-\hat{\gamma}_{k}(t)
$$

- When at least $K_{i j}=2$ simulation chains are available for a $\operatorname{RCM} i$ and a $\operatorname{GCM} j$ (i.e. at least for two emission scenarios), the interaction effect is estimated by:

$$
\hat{\kappa}_{i j}(t)=\frac{1}{K_{i j}} \sum_{k=1}^{K_{i j}} \hat{\xi}_{i, j, k}(t)^{2} .
$$

We can thus estimate $n_{I J}=27 \mathrm{RCM} / \mathrm{GCM}$ interaction effects corresponding to different combinations of RCM and GCM models. Similarly, we estimate $n_{I K}=18 \mathrm{RCM} / \mathrm{RCP}$ interaction effects $\hat{\psi}_{i k}(t)$ and $n_{J K}=19 \mathrm{GCM} / \mathrm{RCP}$ interaction effects $\hat{\omega}_{j k}(t)$. If only one simulation chain is available, the interaction effect is not estimated and is set to zero.

- Finally, remaining residual terms are obtained as:

$$
\hat{\nu}_{i, j, k}(t)=\hat{\xi}_{i, j, k}^{*}(t)-\hat{\kappa}_{i j}(t)-\hat{\psi}_{i k}(t)-\hat{\omega}_{j k}(t) .
$$

It must be acknowledged that these estimates of interaction effects suffer from several limitations. First, these estimates rely on a few simulation chains. Second, we can only estimate a small proportion of all possible interactions (for example $n_{I J}=27$ RCM/GCM interaction effects among the $I \times J=117$ possible GCM/RCM combinations). However, it provides an interesting approximation of the magnitude of these interactions relatively to the other ones.

\subsection{Total variance and variance components}

In this study, we aim at characterizing the total variance and its decomposition as a function of time. For each time $t$, since $Y_{i, j, k}^{*}(t)=\phi_{i, j, k}^{*}(t)+\eta_{i, j, k}^{*}(t)$, if $\phi_{i, j, k}^{*}(t)$ and $\eta_{i, j, k}^{*}(t)$ are assumed to be independent, the total variance of the change variable 
$Y_{i, j, k}^{*}(t)$ is:

$\operatorname{Var}\left[Y_{i, j, k}^{*}(t)\right]=\operatorname{Var}\left[\phi_{i, j, k}^{*}(t)\right]+\operatorname{Var}\left[\eta_{i, j, k}^{*}(t)\right]$

where $\operatorname{Var}\left[\phi_{i, j, k}^{*}(t)\right]$ is the total variance related to the climate change response and $\operatorname{Var}\left[\eta_{i, j, k}^{*}(t)\right]$ characterizes the internal variability of the change variable. Internal variability $\operatorname{Var}\left[\eta_{i, j, k}^{*}(t)\right]$ is considered constant and does not depend on time (see Eq. S10 below).

The variance of the climate change response $\phi_{i, j, k}^{*}(t)$, for any given time $t$, is the sum of the variance of the different uncertainty components in Eq. (S2):

$\operatorname{Var}\left[\phi_{i, j, k}^{*}(t)\right]=\operatorname{Var}\left[\alpha_{i}(t)\right]+\operatorname{Var}\left[\beta_{j}(t)\right]+\operatorname{Var}\left[\gamma_{k}(t)\right]+\operatorname{Var}\left[\xi_{i, j, k}\right]$

where $\operatorname{Var}\left[\alpha_{i}(t)\right], \operatorname{Var}\left[\beta_{j}(t)\right]$ and $\operatorname{Var}\left[\gamma_{k}(t)\right]$ describe the dispersion between the main RCM, GCM and RCP effects and are estimated by:

$$
\begin{aligned}
& \widehat{\operatorname{Var}}\left[\alpha_{i}(t)\right]=\frac{1}{I} \sum_{i}\left[\hat{\alpha}_{i}(t)\right]^{2}, \\
& \widehat{\operatorname{Var}}\left[\beta_{j}(t)\right]=\frac{1}{J} \sum_{j}\left[\hat{\beta}_{j}(t)\right]^{2}, \\
& \widehat{\operatorname{Var}}\left[\gamma_{k}(t)\right]=\frac{1}{K} \sum_{k}\left[\hat{\gamma}_{k}(t)\right]^{2} .
\end{aligned}
$$

The variability $\operatorname{Var}\left[\xi_{i, j, k}\right]$ (so-called residual variability in Evin et al., 2019) is estimated by

$$
\widehat{\operatorname{Var}}[\xi(t)]=\frac{1}{n_{o}} \sum_{\{i, j, k\}^{\circ}} \hat{\xi}_{i, j, k}(t)^{2},
$$

where $\{i, j, k\}^{o}$ is the ensemble of available simulation chains. This variability will be further decompose below using estimates of interaction effects.

For each scenario $k$ and time $t$, the following scenario-excluded uncertainty $B U$ can be used to assess the uncertainty related to the mean expected change $B M_{k}$ :

$$
B U=\sqrt{\widehat{\operatorname{Var}}\left[Y_{i, j, k}^{*}(t)\right]-\widehat{\operatorname{Var}}\left[\gamma_{k}(t)\right]}
$$

Finally, the variability $\operatorname{Var}\left[\xi_{i, j, k}\right]$ is the variability of the climate change response that cannot be explained by the variability of the main RCM, GCM and RCP effects. It is further discomposed into the uncertainty components $\widehat{\operatorname{Var}}\left[\kappa_{i j}(t)\right], \widehat{\operatorname{Var}}\left[\psi_{i k}(t)\right]$, $\widehat{\operatorname{Var}}\left[\omega_{j k}(t)\right]$ corresponding to the interaction effects RCM/GCM, RCM/RCP and GCM/RCP respectively, and a residual variability $\widehat{\mathbb{V a r}}[\nu(t)]$ : 


$$
\begin{aligned}
\widehat{\operatorname{Var}}\left[\kappa_{i j}(t)\right] & =C \times \operatorname{var}\left[\hat{\kappa}_{i j}(t)\right], \\
\widehat{\operatorname{Var}}\left[\psi_{i k}(t)\right] & =C \times \operatorname{var}\left[\hat{\psi}_{i k}(t)\right], \\
\widehat{\operatorname{Var}}\left[\omega_{j k}(t)\right] & =C \times \operatorname{var}\left[\hat{\omega}_{j k}(t)\right], \\
\widehat{\operatorname{Var}}[\nu(t)] & =C \times \operatorname{var}\left[\hat{\nu}_{i, j, k}(t)\right],
\end{aligned}
$$

where var is the standard empirical variance and $C$ is a constant term that weights the different variance terms so that $\widehat{\mathbb{V a r}}\left[\kappa_{i j}(t)\right]+\widehat{\mathbb{V a r}}\left[\psi_{i k}(t)\right]+\widehat{\mathbb{V a r}}\left[\omega_{j k}(t)\right]+\widehat{\operatorname{Var}}[\nu(t)]=\widehat{\mathbb{V a r}}[\xi(t)]$. This scaling is applied in order to preserve the variability $\operatorname{Var}\left[\xi_{i, j, k}\right]$ obtained with QUALYPSO. Indeed, due to the limitations in the estimates of the interaction effects (limited number of terms, unequal number of climate chains for each estimate, not centered on zero), $C$ is generally less than one, and without this scaling, it would result to an inflation of the total uncertainty.

Internal variability of each climate chain corresponds to the variance of the deviations $\eta_{i, j, k}^{*}(t)$ from the climate change response, obtained for each simulation chain. The internal variability component of the ensemble is estimated as the multichain mean (over the $n_{o}$ available chains) of the variances :

$$
\widehat{\operatorname{Var}}\left(\eta_{i, j, k}^{*}\right)=\frac{1}{n_{o}} \sum_{\{i, j, k\}^{\circ}} \frac{1}{n_{t}} \sum_{t} \eta_{i, j, k}^{*}(t)^{2}
$$

where $n_{t}$ corresponds to the length of the simulation chain. In Eq. (S10), internal variability is averaged over time and the resulting estimate $\widehat{\operatorname{Var}}\left(\eta_{i, j, k}^{*}\right)$ is thus considered constant. This assumption could be easily relaxed in case of evidences toward an increase/decrease of the internal variability in the ensemble. 


\section{Mean climate change responses and related uncertainties for the 3 SREX regions.}

Figures S1-S4 show the individual change responses obtained for the different chains (column 1) and the mean change response $B M_{k}$ obtained for each scenario $k$ along with the scenario-excluded uncertainty $B U$ and its different components (columns 2-4), for temperature and precipitation changes in winter and summer. The ensemble of climate change responses is generally very dispersed, and some chains stand out from the others. One chain even simulates a temperature decrease for NEU in winter (see top-left panel in Fig. S1). The mean change response $B M_{k}$ obtained for each scenario $k$ can be put in perspective with the associated uncertainty $B U$ and its different components. For the period 2071-2099, the corresponding $90 \%$ uncertainty bands show important warmings with the RCP8.5, the lower bounds being around $2^{\circ} \mathrm{C}\left([2.0,5.6]^{\circ} \mathrm{C},[2.5,5.1]^{\circ} \mathrm{C}\right.$ and $[2.3,4.0]^{\circ} \mathrm{C}$ in NEU, CEU and MED regions, respectively). In contrast, with the RCP2.6, warmings are moderate $\left([-0.6,3.0]^{\circ} \mathrm{C},[-0.1\right.$, $2.4]^{\circ} \mathrm{C}$ and $[0.3,2.0]^{\circ} \mathrm{C}$ for NEU, CEU and MED regions, respectively). For winter precipitation (Fig. S2), mean changes are rather moderate in comparison of the different uncertainties, in particular GCM and RCM uncertainties. Winter precipitation is projected to increase by $[1 \%, 21 \%]$ in NEU, $[5 \%, 25 \%]$ in CEU and decreases by $[-19 \%, 1 \%]$ in the MED region with the RCP8.5. In summer, with the RCP8.5, the projected warming is slightly more intense than in winter for CEU and MED regions (by about $0.3^{\circ} \mathrm{C}$ ), and more moderate in NEU. For summer precipitation, an important decrease can be noticed in the MED region $([-55 \%, 0 \%])$, with a rather large uncertainty.

For each scenario $k$, the balanced mean changes of the ensemble $B M_{k}$ (white curves) can be compared to the direct averages $M_{k}$ (thick colored curves), see corresponding differences in Tables S18-S19 for temperature and precipitation changes, respectively. These differences are more important in summer (e.g. $0.5^{\circ} \mathrm{C}$ for mean temperature changes in $\mathrm{CEU}$ with the scenarios RCP2.6 and RCP4.5, see Table S18). 

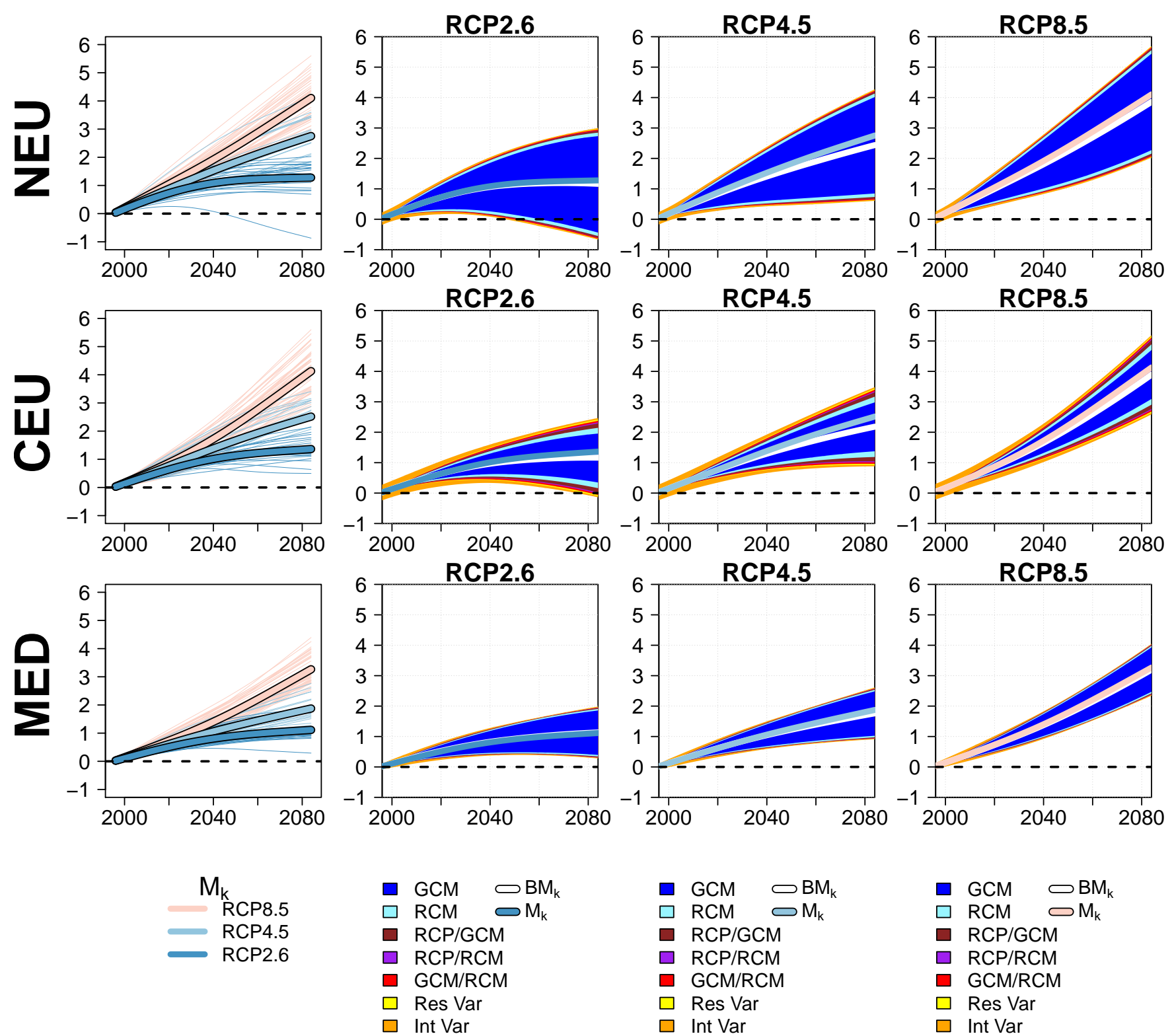

Figure S1. Balanced mean climate change responses $B M_{k}$ of absolute changes of mean temperature in winter (DJF) as a function of time and related uncertainties compared to the period 1981-2010, for the 3 SREX regions. Column 1: Ensembles of climate change responses for each RCP scenario, thick lines represent the direct mean response $M_{k}$, i.e. the direct average of each ensemble. Columns 2-4: For each scenario, the balanced mean change $B M_{k}$ obtained with the QUALYPSO approach (thick white line), can be compared with the direct average $M_{k}$ (thick colored line), as shown in the first column, and corresponding $90 \%$ uncertainty band. The width of the overall colored interval represents the different sources of uncertainty corresponds to $B M_{k} \pm 1.645 B U$. This interval is subdivided into subintervals whose width is proportional to the fraction of total variance explained by the different sources of uncertainties. 

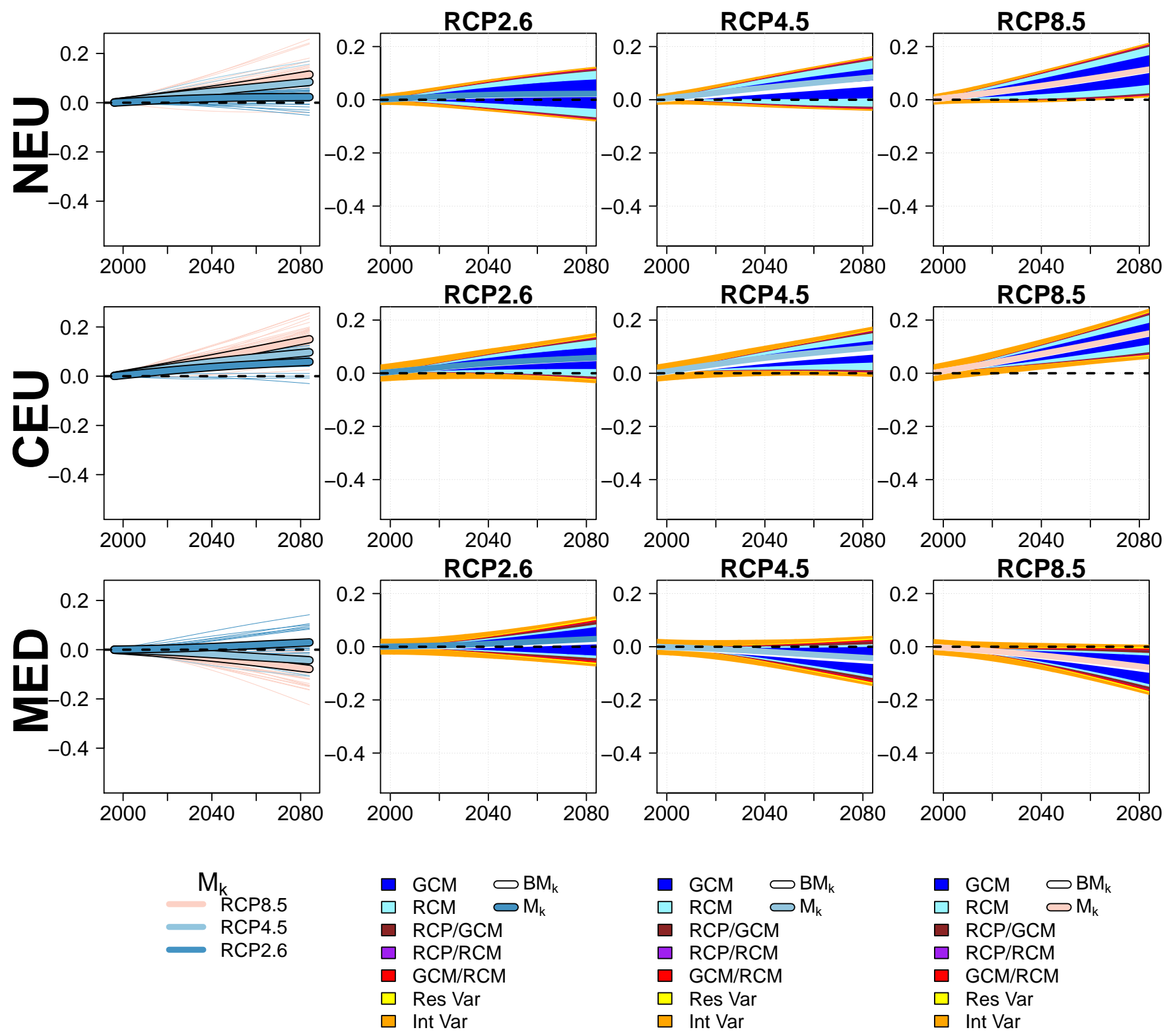

Figure S2. Same as Fig. S1 for relative changes of total precipitation in winter (DJF). 

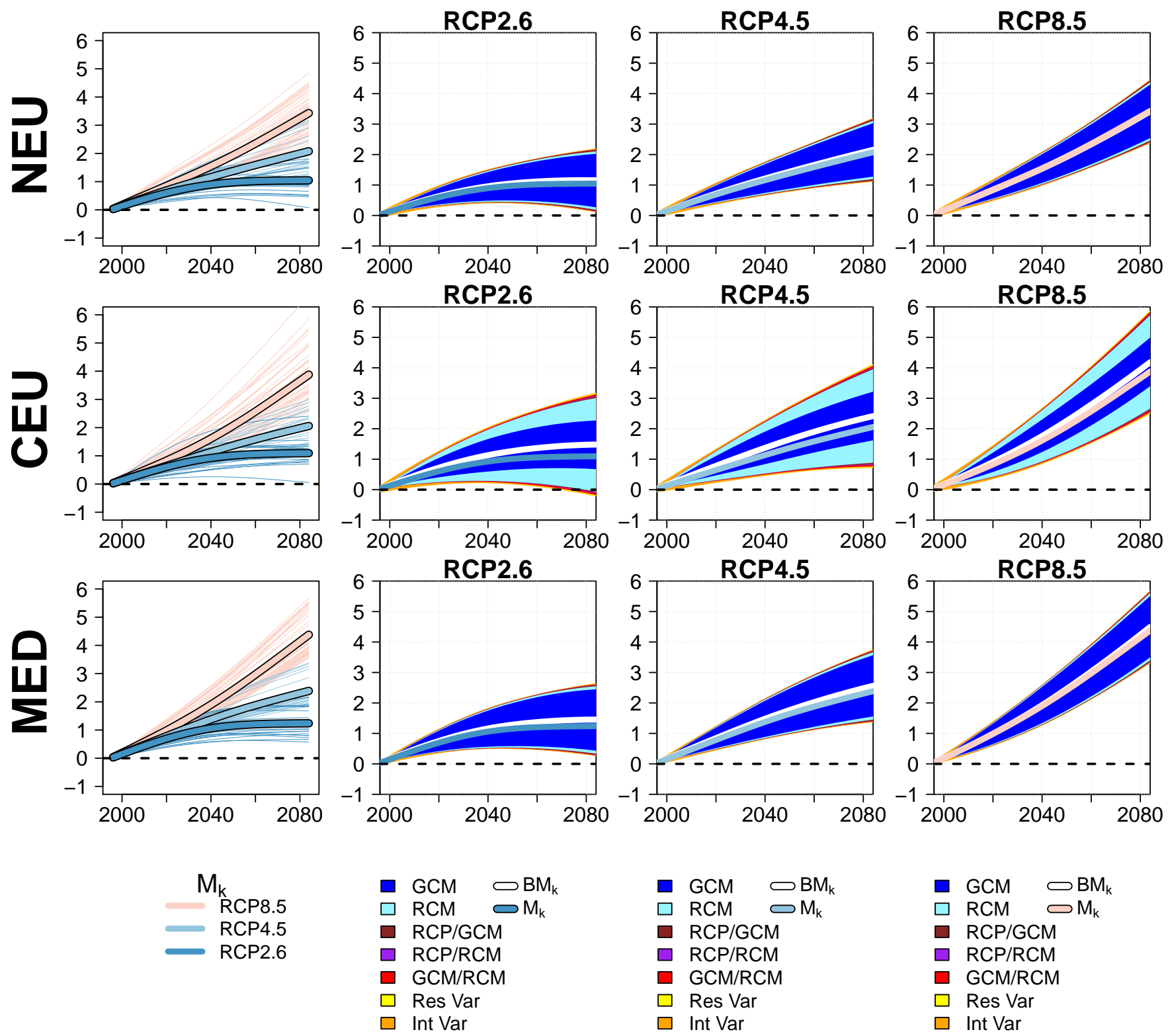

Figure S3. Same as Fig. S1 for absolute changes of mean temperature in summer (JJA). 

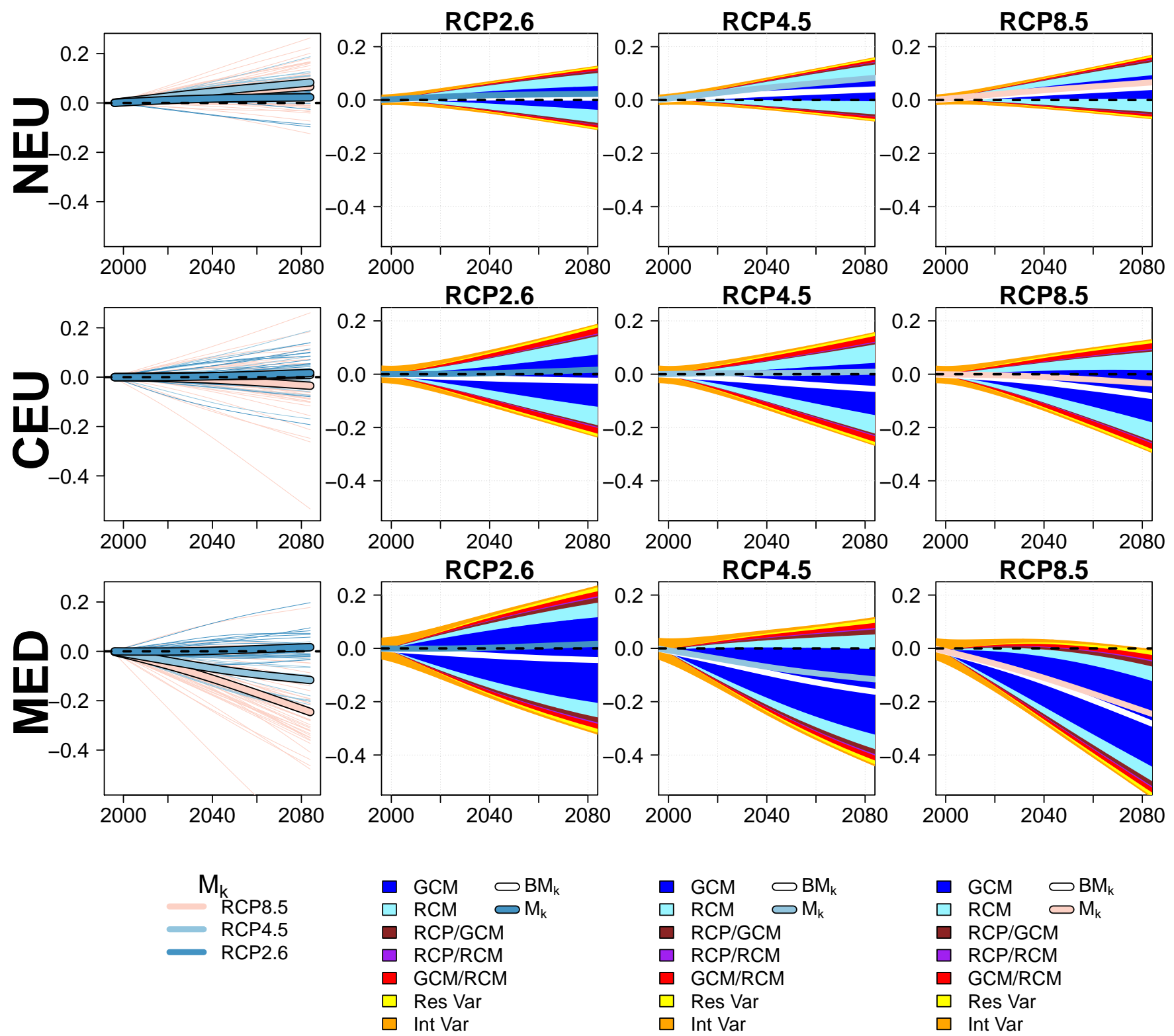

Figure S4. Same as Fig. S1 for relative changes of total precipitation in summer (JJA). 

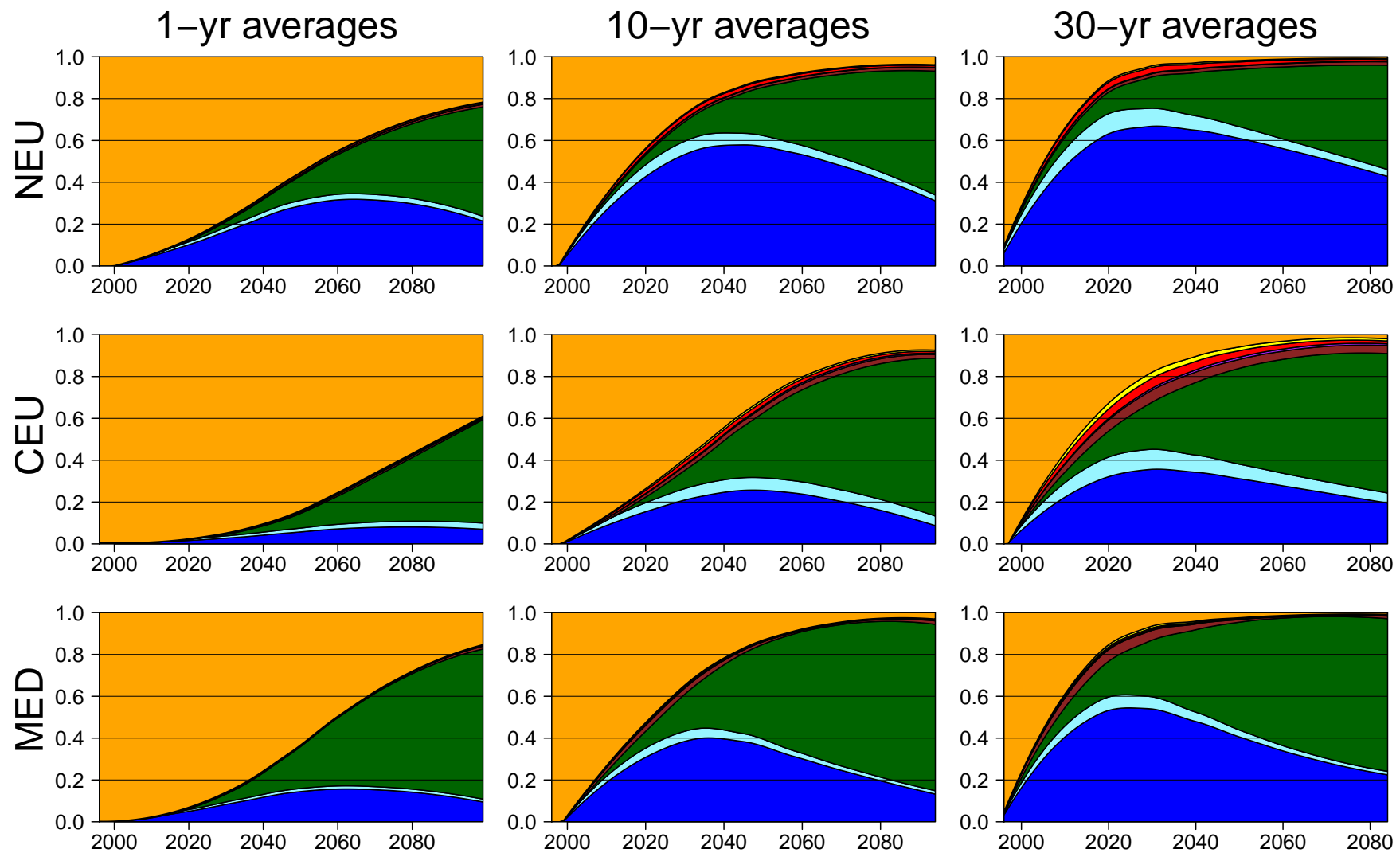

Figure S5. Fraction of total variance for absolute changes of mean temperature in winter (DJF) as a function of time and related uncertainties compared to the period 1981-2010, for the 3 SREX regions and for 1-yr averages (left column), 10-yr averages (middle column) and 30-yr averages (right column). 


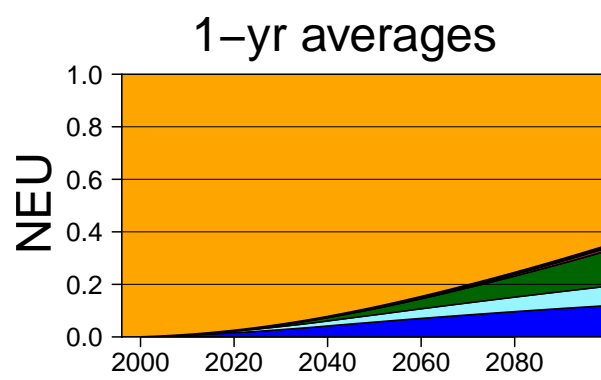

\section{pr / DJF}
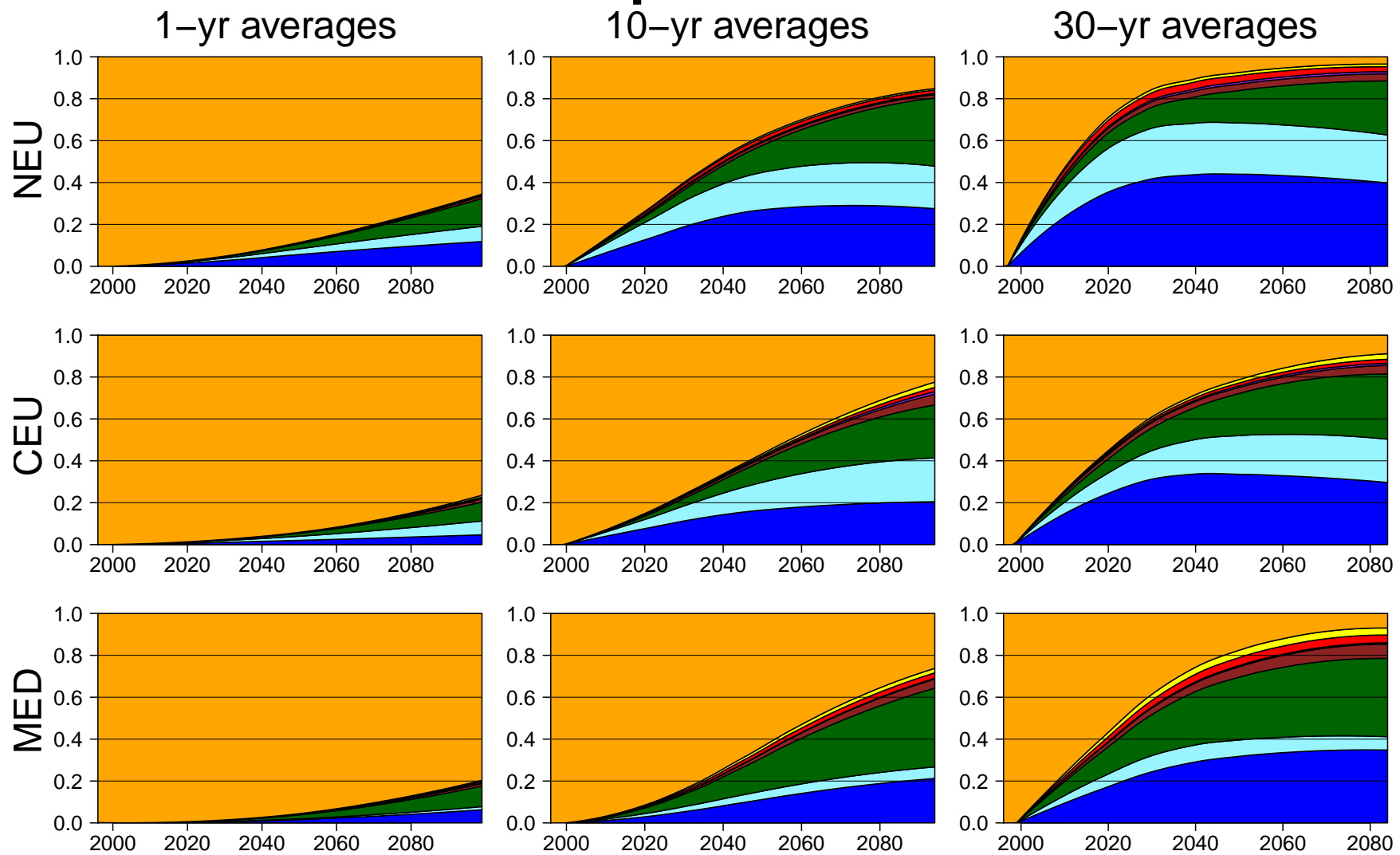

Figure S6. Fraction of total variance for relative changes of total precipitation in winter (DJF) as a function of time and related uncertainties compared to the period 1981-2010, for the 3 SREX regions and for 1-yr averages (left column), 10-yr averages (middle column) and 30-yr averages (right column). 

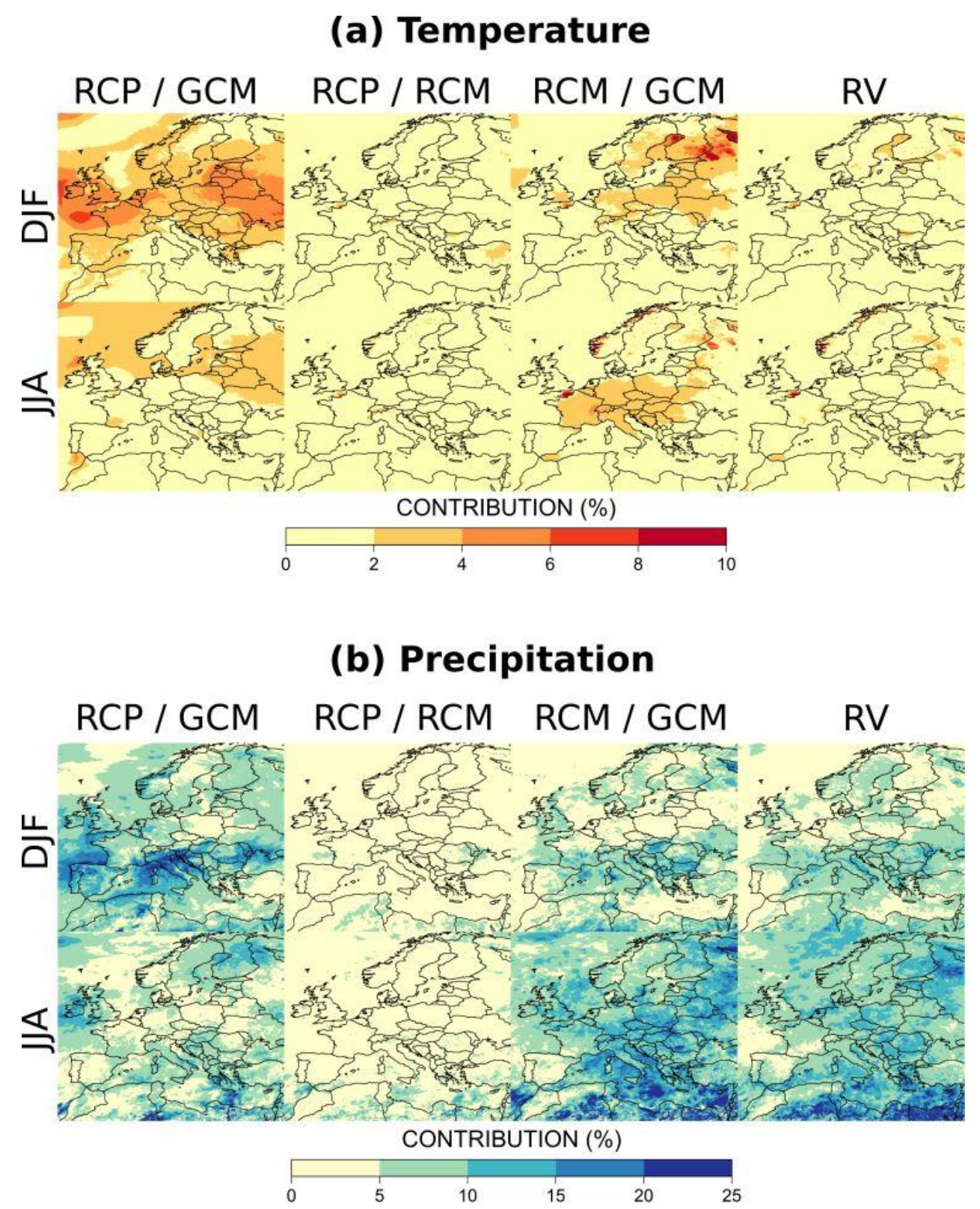

Figure S7. Fraction of total variance explained by interactions: RCP/GCM, RCP/RCM and RCM/GCM, and residual variability (RV) for (a) absolute temperature changes and (b) relative precipitation changes, at the end of the twenty-first century (2071-2099) compared to the period 1981-2010. 
Temperature

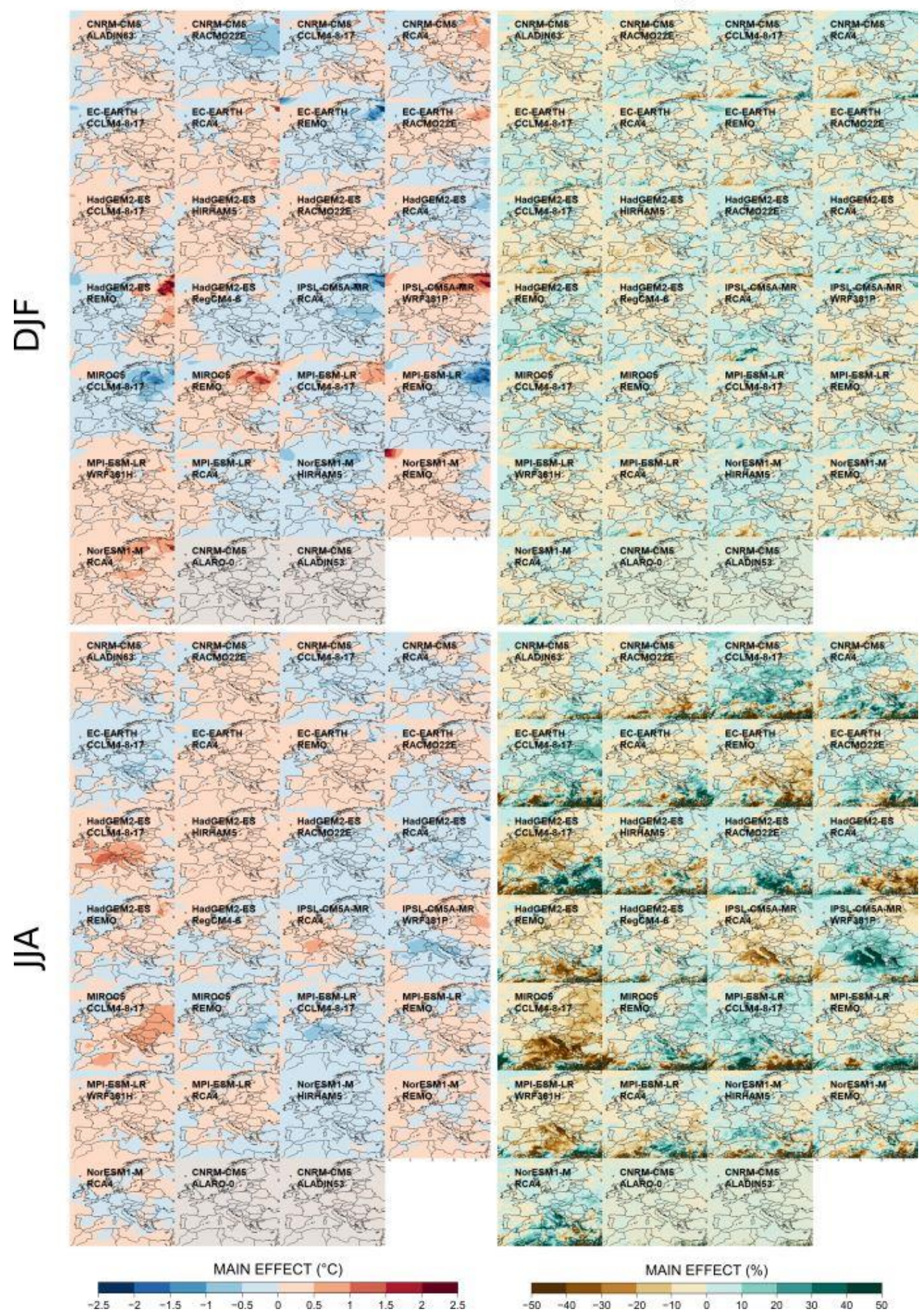

Figure S8. Estimated GCM/RCM interaction effects for absolute temperature (left) and relative precipitation changes (right) in winter (DJF) and summer (JJA), at the end of the twenty-first century (2071-2099) compared to the period 1981-2010. 


\section{Temperature}

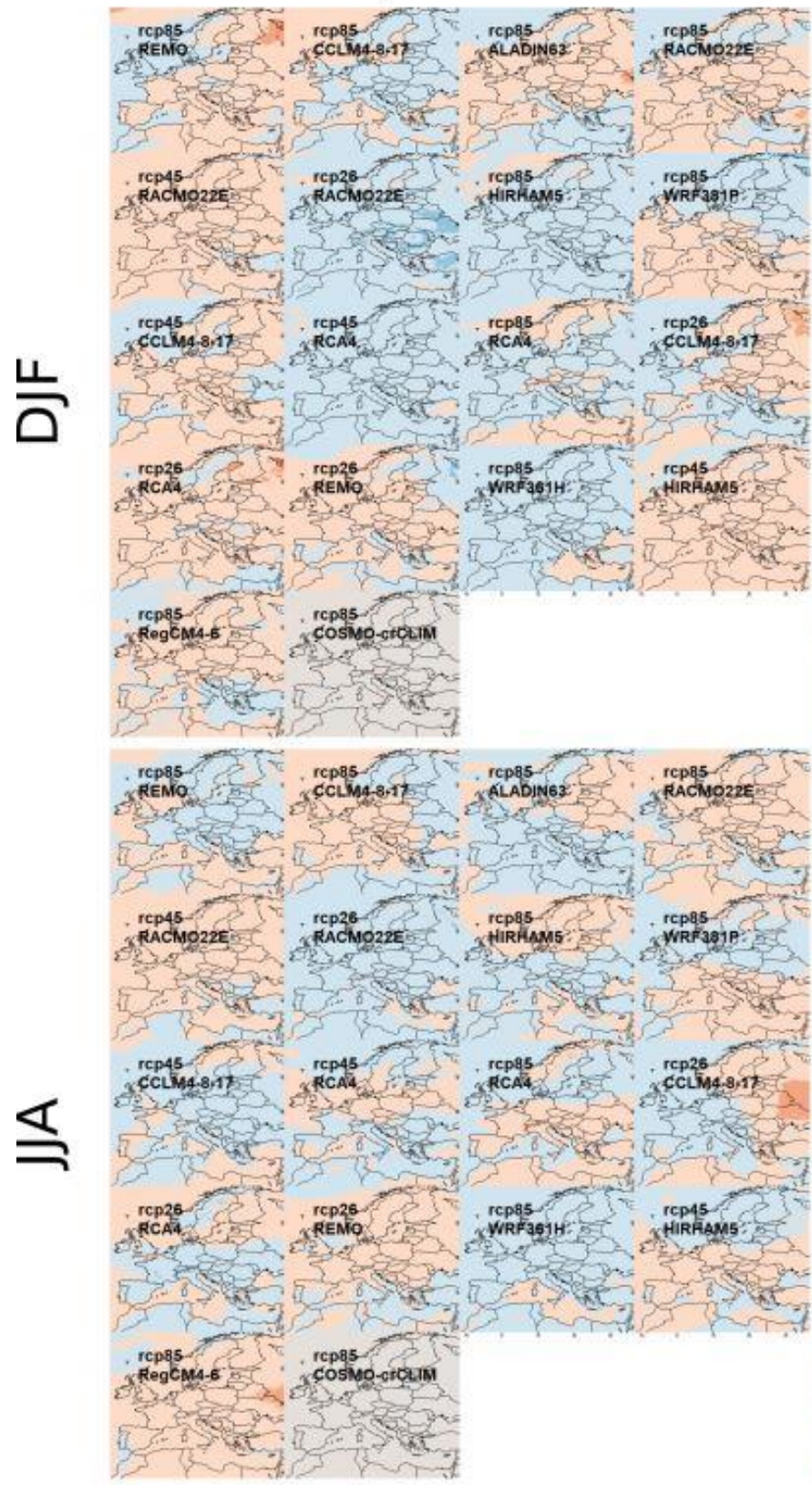

MAIN EFFECT $\left({ }^{\circ} \mathrm{C}\right)$

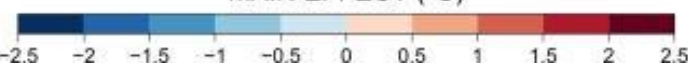

\section{Precipitation}
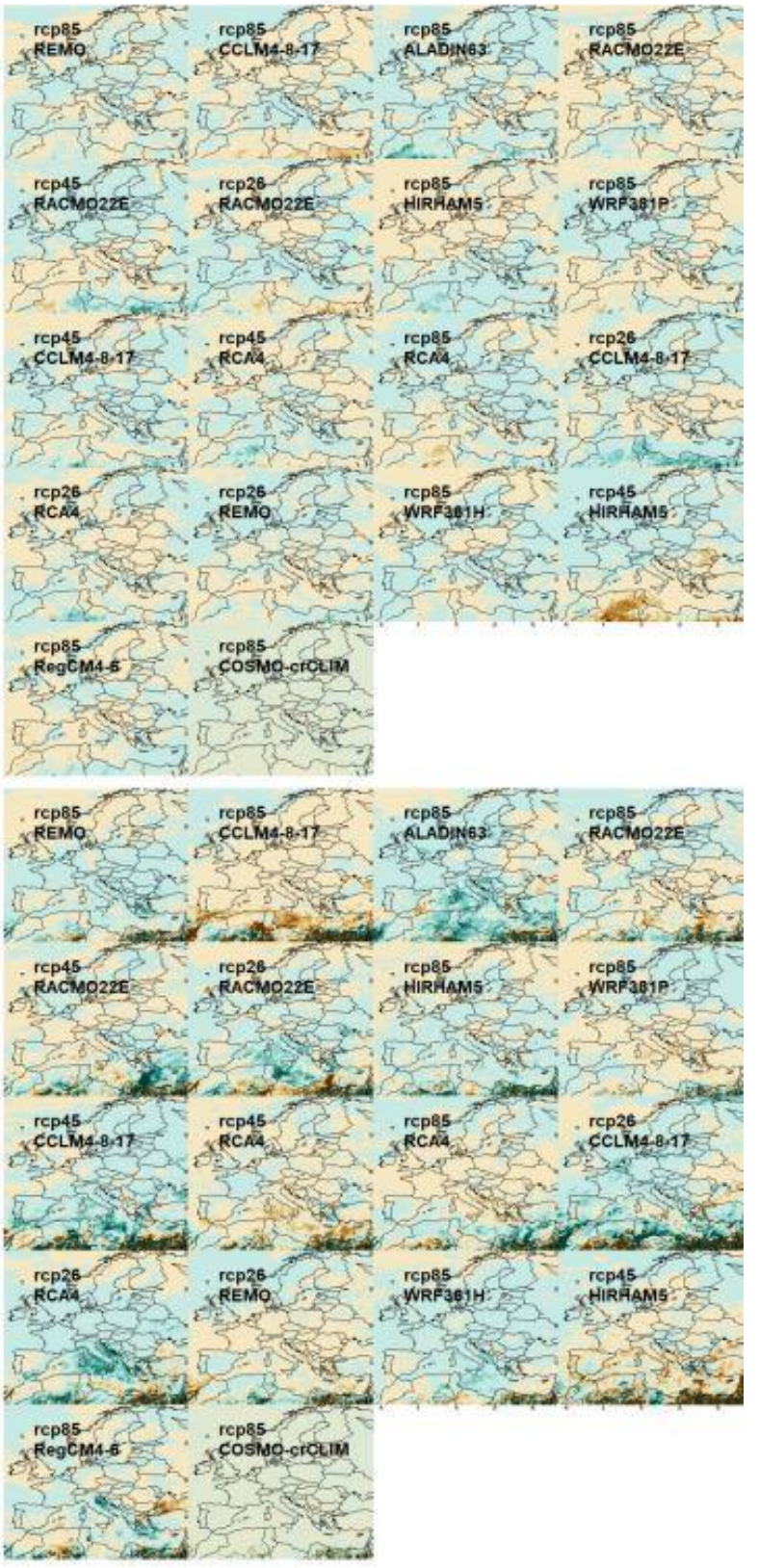

MAIN EFFECT (\%)

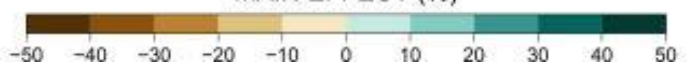

Figure S9. Estimated RCP/RCM interaction effects for absolute temperature (left) and relative precipitation changes (right) in winter (DJF) and summer (JJA), at the end of the twenty-first century (2071-2099) compared to the period 1981-2010. 


\section{Temperature}
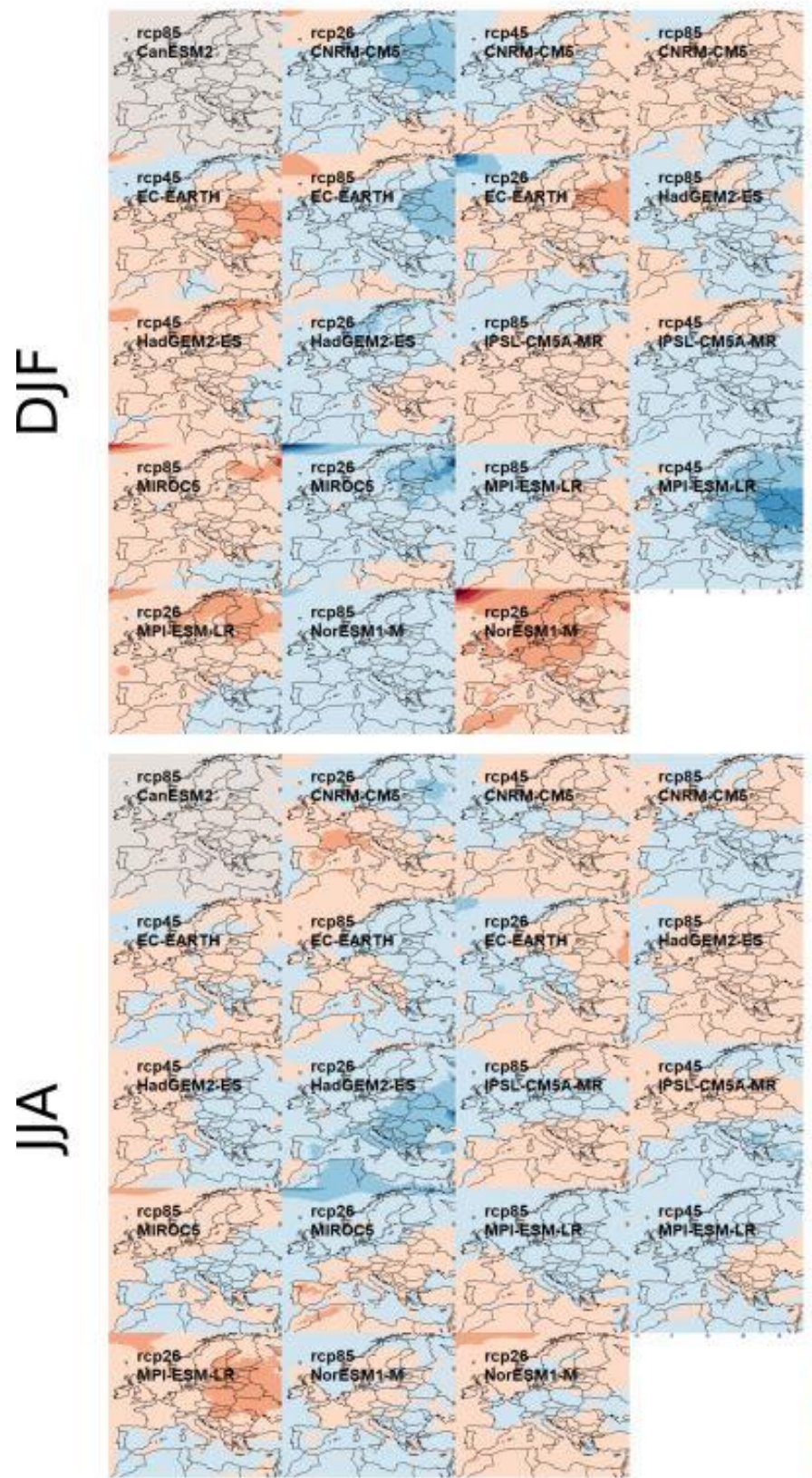

MAIN EFFECT $\left({ }^{\circ} \mathrm{C}\right)$

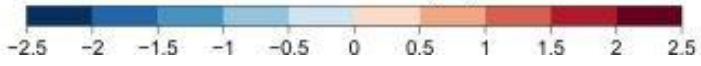

\section{Precipitation}
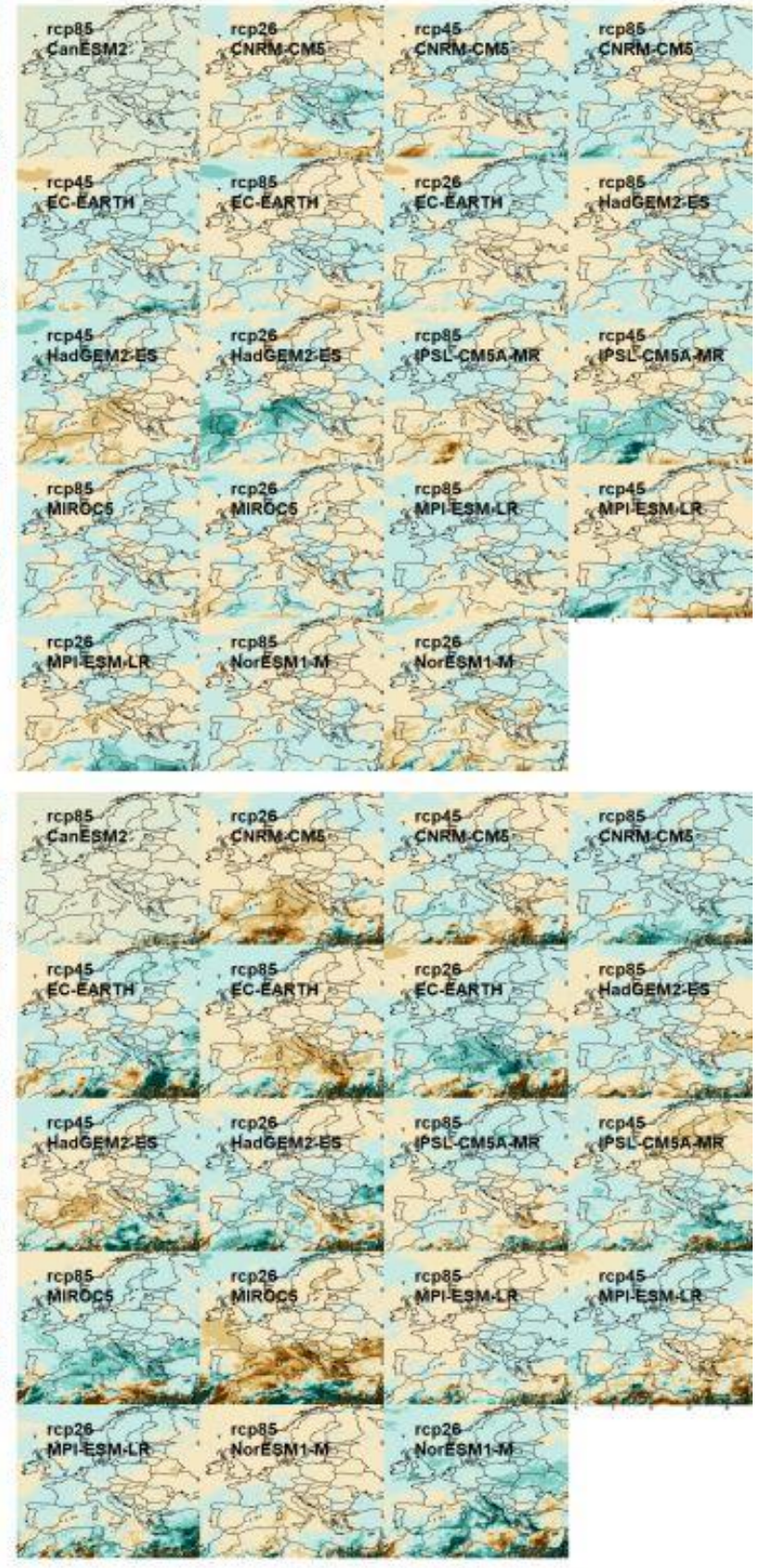

MAIN EFFECT (\%)

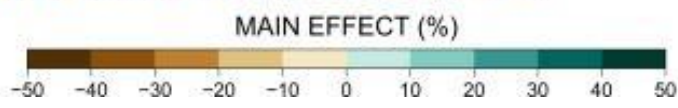

Figure S10. Estimated RCP/GCM interaction effects for absolute temperature (left) and relative precipitation changes (right) in winter (DJF) and summer (JJA), at the end of the twenty-first century (2071-2099) compared to the period 1981-2010. 


\section{Main results for the countries of the domain}

Table S2. Mean expected change response $B M_{k}$, scenario-excluded uncertainty $B U$ and partition of the total uncertainty for the countries of the domain, for absolute changes of mean temperature in winter (DJF) in the near future (2021-2050) compared to the period 1981-2010. I1, I2 and I3 refer to interactions RCM/GCM, RCP/RCM and RCP/GCM, respectively. IV and RV refer to internal and residual variability, respectively. Absolute mean expected changes greater than the uncertainty $(|B U / B M|>1)$ are indicated in bold font.

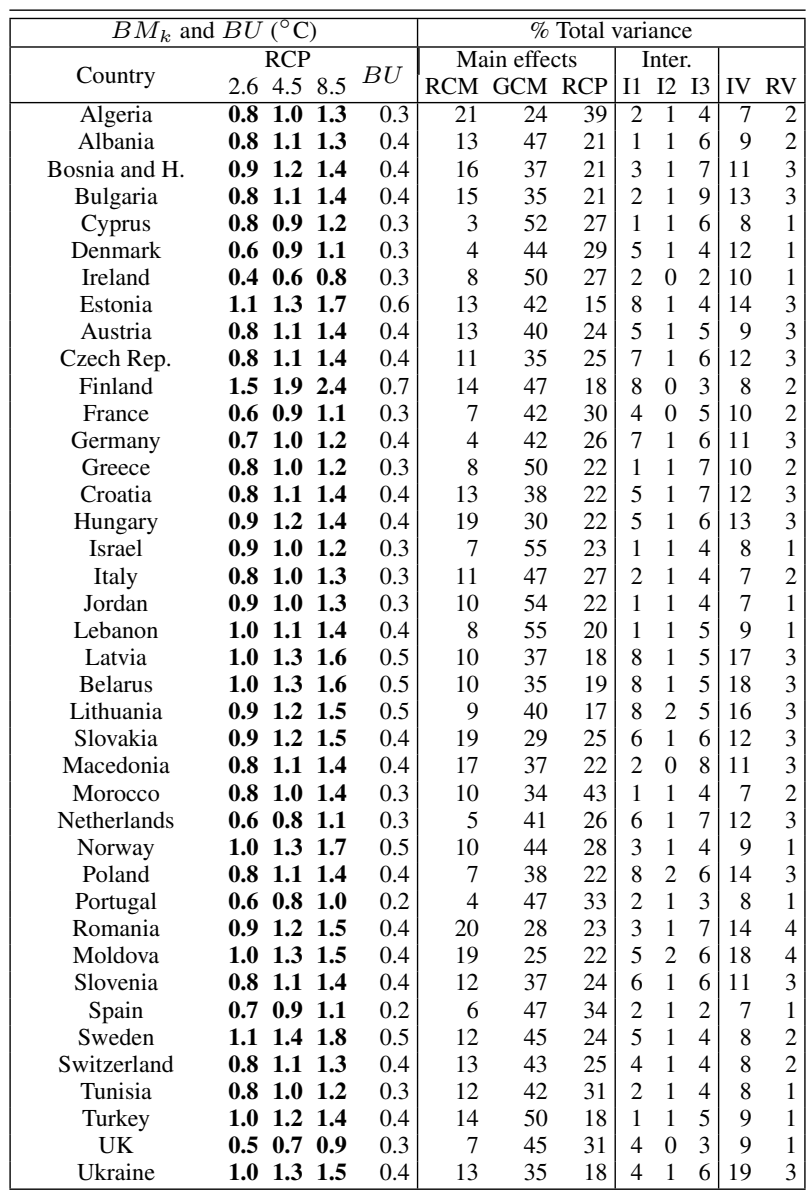


Table S3. Absolute changes of mean temperature in winter (DJF) at the end of the century (2071-2099) compared to the period 1981-2010 (see legend of Table S2 for further details).

\begin{tabular}{|c|c|c|c|c|c|c|c|c|c|c|}
\hline \multicolumn{3}{|c|}{$B M_{k}$ and $B U\left({ }^{\circ} \mathrm{C}\right)$} & \multicolumn{8}{|c|}{$\%$ Total variance } \\
\hline \multirow{2}{*}{ Country } & $\mathrm{RCP}$ & \multirow{2}{*}{$B U$} & \multicolumn{3}{|c|}{ Main effects } & \multicolumn{3}{|c|}{ Inter. } & \multirow[b]{2}{*}{ IV } & \\
\hline & $\begin{array}{lll}2.6 & 4.5 & 8.5\end{array}$ & & RCM & GCM & RCP & I1 & & I3 & & RV \\
\hline Algeria & $\begin{array}{lll}1.2 & 1.8 & 3.5\end{array}$ & 0.6 & 6 & 15 & 75 & 1 & 1 & & 1 & 1 \\
\hline Albania & $\begin{array}{lll}1.3 & 2.0 & 3.4\end{array}$ & 0.8 & 5 & 33 & 57 & 1 & 1 & 1 & 1 & 1 \\
\hline Bosnia and $\mathrm{H}$. & $\begin{array}{lll}1.4 & 2.2 & 3.7\end{array}$ & 0.8 & 5 & 31 & 56 & 1 & 1 & 2 & 2 & 1 \\
\hline Bulgaria & $\begin{array}{lll}1.4 & 2.1 & 3.7\end{array}$ & 0.8 & 6 & 26 & 60 & 1 & 1 & 3 & 2 & 1 \\
\hline Cyprus & $\begin{array}{lll}1.2 & 1.8 & 3.3\end{array}$ & 0.6 & 1 & 29 & 67 & 0 & 0 & 1 & 1 & 0 \\
\hline Denmark & $\begin{array}{lll}0.7 & 1.8 & 3.1\end{array}$ & 0.7 & 2 & 22 & 68 & 2 & 0 & 4 & 1 & 1 \\
\hline Ireland & $\begin{array}{lll}0.4 & 1.2 & 2.2\end{array}$ & 0.5 & 3 & 17 & 70 & 2 & 1 & 5 & 1 & 1 \\
\hline Estonia & $\begin{array}{lll}1.2 & 2.7 & 4.5\end{array}$ & 1.3 & 7 & 31 & 52 & 2 & 1 & 4 & 2 & 2 \\
\hline Austria & 1.12 .13 .6 & 0.8 & 5 & 22 & 65 & 2 & 1 & 2 & 1 & 1 \\
\hline Czech Rep. & $\begin{array}{lll}1.1 & 2.1 & 3.7\end{array}$ & 0.8 & 6 & 21 & 64 & 2 & 1 & 3 & 1 & 2 \\
\hline Finland & $\begin{array}{lll}1.9 & 3.6 & 5.7\end{array}$ & 1.6 & 8 & 38 & 47 & 2 & 0 & 2 & 1 & 1 \\
\hline France & $\begin{array}{lll}0.8 & 1.6 & 2.9\end{array}$ & 0.5 & 3 & 16 & 73 & 1 & 1 & 4 & 1 & 1 \\
\hline Germany & $\begin{array}{lll}0.9 & 1.9 & 3.3\end{array}$ & 0.7 & 3 & 22 & 66 & 2 & 1 & 4 & 1 & 1 \\
\hline Greece & $\begin{array}{lll}1.3 & 1.9 & 3.3\end{array}$ & 0.7 & 3 & 33 & 60 & 0 & 1 & 2 & 1 & 1 \\
\hline Croatia & $\begin{array}{lll}1.3 & 2.1 & 3.6\end{array}$ & 0.8 & 5 & 27 & 60 & 1 & 1 & 3 & 2 & 1 \\
\hline Hungary & $\begin{array}{lll}1.3 & 2.2 & 3.7\end{array}$ & 0.8 & 8 & 24 & 59 & 2 & 1 & 3 & 2 & 2 \\
\hline Israel & $\begin{array}{lll}1.4 & 1.9 & 3.5\end{array}$ & 0.7 & 3 & 31 & 63 & 0 & 0 & 1 & 1 & 1 \\
\hline Italy & $\begin{array}{lll}1.1 & 2.0 & 3.4\end{array}$ & 0.6 & 3 & 25 & 67 & 1 & 1 & 1 & 1 & 1 \\
\hline Jordan & $\begin{array}{lll}1.5 & 2.0 & 3.8\end{array}$ & 0.7 & 4 & 29 & 64 & 0 & 0 & 1 & 1 & 1 \\
\hline Lebanon & $\begin{array}{lll}1.5 & 2.2 & 3.8\end{array}$ & 0.8 & 3 & 34 & 59 & 0 & 0 & 1 & 1 & 1 \\
\hline Latvia & $\begin{array}{lll}1.1 & 2.5 & 4.4\end{array}$ & 1.2 & 6 & 29 & 54 & 2 & 1 & 5 & 2 & 2 \\
\hline Belarus & $\begin{array}{lll}1.2 & 2.5 & 4.5\end{array}$ & 1.2 & 8 & 25 & 57 & 2 & 1 & 4 & 2 & 2 \\
\hline Lithuania & $\begin{array}{lll}1.1 & 2.4 & 4.3\end{array}$ & 1.2 & 6 & 28 & 55 & 2 & 1 & 5 & 2 & 2 \\
\hline Slovakia & $\begin{array}{lll}1.2 & 2.2 & 3.8\end{array}$ & 0.9 & 8 & 23 & 61 & 2 & 1 & 2 & 1 & 2 \\
\hline Macedonia & 1.42 .13 .6 & 0.8 & 7 & 28 & 59 & 1 & 1 & 2 & 1 & 1 \\
\hline Morocco & $\begin{array}{lll}1.1 & 1.9 & 3.6\end{array}$ & 0.5 & 4 & 11 & 81 & 0 & 0 & 2 & 1 & 1 \\
\hline Netherlands & $\begin{array}{lll}0.7 & 1.6 & 2.9\end{array}$ & 0.6 & 3 & 17 & 70 & 2 & 1 & 4 & 1 & 1 \\
\hline Norway & $\begin{array}{lll}1.2 & 2.6 & 4.3\end{array}$ & 1.0 & 6 & 29 & 60 & 1 & 0 & 2 & 1 & 1 \\
\hline Poland & $\begin{array}{lll}1.0 & 2.1 & 3.8\end{array}$ & 1.0 & 5 & 26 & 59 & 2 & 1 & 4 & 2 & 2 \\
\hline Portugal & $\begin{array}{lll}0.7 & 1.4 & 2.6\end{array}$ & 0.4 & 2 & 14 & 79 & 1 & 0 & 2 & 1 & 1 \\
\hline Romania & $\begin{array}{lll}1.4 & 2.2 & 3.9\end{array}$ & 0.8 & 9 & 22 & 61 & 1 & 1 & 3 & 2 & 1 \\
\hline Moldova & $\begin{array}{lll}1.5 & 2.4 & 4.2\end{array}$ & 0.9 & 11 & 19 & 59 & 2 & 1 & 4 & 2 & 2 \\
\hline Slovenia & 1.12 .13 .6 & 0.8 & 5 & 22 & 64 & & 1 & 3 & 1 & 1 \\
\hline Spain & $\begin{array}{lll}0.8 & 1.6 & 2.9\end{array}$ & 0.4 & 3 & 13 & 80 & 1 & 0 & 2 & 1 & 1 \\
\hline Sweden & $\begin{array}{lll}1.3 & 2.7 & 4.6\end{array}$ & 1.2 & 6 & 33 & 55 & 2 & 0 & 2 & 1 & 1 \\
\hline Switzerland & $\begin{array}{lll}1.0 & 2.1 & 3.5\end{array}$ & 0.7 & 6 & 19 & 68 & 2 & 1 & 3 & 1 & 1 \\
\hline Tunisia & $\begin{array}{lll}1.2 & 1.8 & 3.3\end{array}$ & 0.6 & 4 & 23 & 69 & 1 & 0 & 1 & 1 & 1 \\
\hline Turkey & $\begin{array}{lll}1.6 & 2.2 & 4.0\end{array}$ & 0.8 & 7 & 27 & 61 & 1 & 1 & 1 & 1 & 1 \\
\hline UK & $\begin{array}{llll}0.5 & 1.4 & 2.4\end{array}$ & 0.5 & 3 & 17 & 71 & 2 & 1 & 4 & 1 & 1 \\
\hline Ukraine & $\begin{array}{lll}1.5 & 2.4 & 4.3 \\
\end{array}$ & 0.9 & 9 & 17 & 62 & 2 & 1 & 5 & 2 & 1 \\
\hline
\end{tabular}


Table S4. Relative changes of total precipitation in winter (DJF) in the near future (2021-2050) compared to the period 1981-2010 (see legend of Table S2 for further details).

\begin{tabular}{|c|c|c|c|c|c|c|c|c|c|c|c|c|}
\hline \multicolumn{5}{|c|}{$B M_{k}$ and $B U(\%)$} & \multicolumn{8}{|c|}{$\%$ Total variance } \\
\hline \multirow{2}{*}{ Country } & \multicolumn{3}{|c|}{$\mathrm{RCP}$} & \multirow{2}{*}{$B U$} & \multicolumn{3}{|c|}{ Main effects } & \multicolumn{3}{|c|}{ Inter. } & \multirow[b]{2}{*}{ IV } & \multirow[b]{2}{*}{ RV } \\
\hline & 2.6 & 4.5 & 8.5 & & $\mathrm{RCM}$ & GCM & $\mathrm{RCP}$ & & $\mathrm{I} 2 \mathrm{I}$ & I3 & & \\
\hline Algeria & -6 & -8 & -14 & 6 & 10 & 25 & 23 & 2 & 1 & 3 & 34 & 2 \\
\hline Albania & 4 & 1 & 1 & 5 & 10 & 28 & 7 & 6 & 1 & 7 & 38 & 4 \\
\hline Bosnia and $\mathrm{H}$. & 4 & 4 & 3 & 5 & 20 & 23 & 1 & 5 & 1 & 7 & 40 & 3 \\
\hline Bulgaria & 3 & 1 & 3 & 5 & 16 & 22 & 2 & 8 & 1 & 8 & 38 & 5 \\
\hline Cyprus & -4 & -6 & -9 & 7 & 12 & 38 & 10 & 5 & 1 & 5 & 25 & 4 \\
\hline Denmark & 2 & 5 & 7 & 5 & 28 & 18 & 15 & 4 & 1 & 3 & 28 & 3 \\
\hline Ireland & 1 & 2 & 5 & 4 & 36 & 16 & 11 & 4 & 2 & 9 & 19 & 3 \\
\hline Estonia & 1 & 3 & 6 & 5 & 30 & 20 & 13 & 4 & 2 & 3 & 24 & 3 \\
\hline Austria & 3 & 3 & 6 & 5 & 17 & 43 & 5 & 3 & 1 & 3 & 24 & 4 \\
\hline Czech Rep. & 3 & 5 & 8 & 6 & 27 & 33 & 13 & 2 & 1 & 3 & 19 & 2 \\
\hline Finland & 3 & 4 & 7 & 5 & 26 & 24 & 12 & 5 & 1 & 4 & 25 & 2 \\
\hline France & 3 & 4 & 5 & 3 & 14 & 17 & 2 & 2 & 1 & 4 & 58 & 2 \\
\hline Germany & 2 & 4 & 7 & 5 & 18 & 28 & 13 & 2 & 1 & 6 & 30 & 2 \\
\hline Greece & 1 & -2 & -2 & 5 & 11 & 38 & 7 & 2 & 1 & 4 & 35 & 3 \\
\hline Croatia & 6 & 5 & 5 & 5 & 10 & 18 & 1 & 7 & 11 & 13 & 47 & 4 \\
\hline Hungary & 5 & 7 & 8 & 6 & 12 & 33 & 4 & 8 & 1 & 5 & 33 & 4 \\
\hline Israel & -5 & -5 & -7 & 7 & 21 & 28 & 2 & 5 & 1 & 9 & 31 & 3 \\
\hline Italy & 5 & 2 & 1 & 5 & 6 & 17 & 12 & 6 & 1 & 10 & 44 & 4 \\
\hline Jordan & -6 & -7 & -10 & 8 & 13 & 22 & 6 & 6 & 21 & 10 & 38 & 4 \\
\hline Lebanon & -4 & -6 & -8 & 7 & 14 & 36 & 4 & 4 & 1 & 7 & 32 & 3 \\
\hline Latvia & 2 & 3 & 6 & 5 & 25 & 22 & 14 & 3 & 2 & 3 & 27 & 3 \\
\hline Belarus & 3 & 4 & 7 & 4 & 18 & 30 & 18 & 2 & 0 & 2 & 28 & 2 \\
\hline Lithuania & 2 & 4 & 7 & 4 & 22 & 20 & 18 & 3 & 2 & 2 & 30 & 4 \\
\hline Slovakia & 5 & 6 & 8 & 5 & 16 & 28 & 6 & 5 & 1 & 3 & 37 & 3 \\
\hline Macedonia & 3 & 1 & 2 & 5 & 19 & 25 & 2 & 6 & 1 & 5 & 39 & 4 \\
\hline Morocco & -4 & -11 & -18 & 8 & 17 & 13 & 33 & 2 & 0 & 5 & 30 & 1 \\
\hline Netherlands & 3 & 4 & 7 & 5 & 25 & 18 & 13 & 3 & 1 & 4 & 33 & 2 \\
\hline Norway & 0 & 1 & 4 & 5 & 29 & 27 & 9 & 3 & 1 & 4 & 24 & 2 \\
\hline Poland & 2 & 4 & 8 & 5 & 20 & 37 & 18 & 1 & 1 & 2 & 20 & 2 \\
\hline Portugal & 2 & 0 & -4 & 6 & 8 & 23 & 14 & 3 & 1 & 7 & 42 & 2 \\
\hline Romania & 4 & 5 & 6 & 5 & 24 & 29 & 3 & 3 & 1 & 7 & 27 & 5 \\
\hline Moldova & 4 & 3 & 7 & 7 & 13 & 28 & 5 & 3 & 3 & 6 & 35 & 6 \\
\hline Slovenia & 7 & 5 & 6 & 6 & 12 & 16 & 3 & 5 & 1 & 12 & 48 & 4 \\
\hline Spain & 1 & -1 & -4 & 5 & 12 & 15 & 17 & 3 & 1 & 9 & 41 & 2 \\
\hline Sweden & 2 & 4 & 7 & 4 & 26 & 16 & 25 & 4 & 1 & 2 & 23 & 3 \\
\hline Switzerland & 4 & 5 & 6 & 5 & 9 & 28 & 3 & 4 & 1 & 7 & 44 & 3 \\
\hline Tunisia & -5 & -8 & -9 & 8 & 14 & 41 & 6 & 3 & 2 & 2 & 30 & 4 \\
\hline Turkey & 0 & -2 & -1 & 5 & 8 & 54 & 2 & 3 & 0 & 4 & 26 & 3 \\
\hline UK & 1 & 3 & 5 & 4 & 38 & 11 & 14 & 4 & 2 & 7 & 21 & 2 \\
\hline Ukraine & 3 & 4 & 6 & 5 & 9 & 42 & 7 & 4 & 1 & 6 & 29 & 3 \\
\hline
\end{tabular}


Table S5. Relative changes of total precipitation in winter (DJF) at the end of the century (2071-2099) compared to the period 1981-2010 (see legend of Table S2 for further details).

\begin{tabular}{|c|c|c|c|c|c|c|c|c|c|c|c|c|}
\hline \multicolumn{5}{|c|}{$B M_{k}$ and $B U(\%)$} & \multicolumn{8}{|c|}{$\%$ Total variance } \\
\hline \multirow{2}{*}{ Country } & \multicolumn{3}{|c|}{$\mathrm{RCP}$} & \multirow[b]{2}{*}{$B U$} & \multicolumn{3}{|c|}{ Main effects } & \multicolumn{3}{|c|}{ Inter. } & \multirow[b]{2}{*}{ IV } & \multirow[b]{2}{*}{ RV } \\
\hline & 2.6 & 4.5 & 8.5 & & $\mathrm{RCM}$ & GCM & $\mathrm{RCP}$ & & & I3 & & \\
\hline Algeria & -8 & -17 & -31 & 11 & 10 & 21 & 46 & 3 & 3 & 8 & 8 & 2 \\
\hline Albania & 7 & 0 & 1 & 9 & 18 & 26 & 9 & 11 & 1 & 16 & 12 & 7 \\
\hline Bosnia and $\mathrm{H}$. & 9 & 7 & 9 & 9 & 27 & 21 & 2 & 10 & 1 & 20 & 12 & 7 \\
\hline Bulgaria & 6 & 1 & 8 & 11 & 20 & 33 & 6 & 12 & 2 & 11 & 10 & 7 \\
\hline Cyprus & -9 & -15 & -24 & 14 & 14 & 51 & 17 & 4 & 2 & 4 & 5 & 4 \\
\hline Denmark & 2 & 10 & 18 & 10 & 35 & 16 & 29 & 4 & 2 & 6 & 5 & 3 \\
\hline Ireland & 1 & 6 & 12 & 9 & 34 & 23 & 21 & 3 & 2 & 11 & 4 & 3 \\
\hline Estonia & 1 & 7 & 17 & 10 & 31 & 17 & 31 & 3 & 2 & 7 & 5 & 4 \\
\hline Austria & 5 & 7 & 13 & 9 & 17 & 50 & 13 & 3 & 2 & 2 & 7 & 6 \\
\hline Czech Rep. & 3 & 9 & 18 & 10 & 24 & 34 & 27 & 2 & 1 & 4 & 5 & 3 \\
\hline Finland & 5 & 10 & 20 & 10 & 24 & 27 & 28 & 4 & 2 & 7 & 5 & 3 \\
\hline France & 6 & 6 & 11 & 6 & 26 & 25 & 11 & 4 & 2 & 7 & 19 & 6 \\
\hline Germany & 3 & 9 & 17 & 8 & 22 & 22 & 36 & 2 & 1 & 8 & 8 & 2 \\
\hline Greece & 2 & -7 & -6 & 10 & 14 & 48 & 13 & 3 & 1 & 8 & 9 & 5 \\
\hline Croatia & 13 & 10 & 13 & 10 & 18 & 18 & 3 & 11 & 2 & 27 & 12 & 9 \\
\hline Hungary & 11 & 12 & 20 & 11 & 16 & 31 & 12 & 11 & 2 & 13 & 8 & 8 \\
\hline Israel & -12 & -10 & -21 & 13 & 17 & 48 & 11 & 4 & 2 & 7 & 8 & 3 \\
\hline Italy & 12 & 2 & 3 & 10 & 12 & 21 & 20 & 8 & 2 & 21 & 10 & 7 \\
\hline Jordan & -14 & -14 & -27 & 15 & 12 & 43 & 14 & 6 & 2 & 9 & 10 & 4 \\
\hline Lebanon & -11 & -13 & -22 & 14 & 14 & 55 & 11 & 3 & 1 & 5 & 8 & 3 \\
\hline Latvia & 2 & 8 & 17 & 9 & 29 & 18 & 33 & 3 & 2 & 7 & 5 & 3 \\
\hline Belarus & 5 & 10 & 19 & 8 & 23 & 25 & 36 & 2 & 1 & 5 & 6 & 3 \\
\hline Lithuania & 3 & 9 & 18 & 8 & 26 & 18 & 35 & 2 & 1 & 5 & 6 & 5 \\
\hline Slovakia & 9 & 10 & 17 & 10 & 18 & 38 & 13 & 6 & 2 & 7 & 9 & 6 \\
\hline Macedonia & 5 & -1 & 4 & 9 & 26 & 26 & 7 & 9 & 1 & 11 & 12 & 9 \\
\hline Morocco & -3 & -24 & -41 & 14 & 14 & 13 & 56 & 2 & 1 & 6 & 7 & 1 \\
\hline Netherlands & 4 & 8 & 19 & 9 & 28 & 18 & 32 & 3 & 1 & 8 & 8 & 2 \\
\hline Norway & -1 & 3 & 10 & 11 & 28 & 35 & 17 & 3 & 1 & 6 & 5 & 3 \\
\hline Poland & 2 & 9 & 17 & 9 & 22 & 35 & 31 & 1 & 1 & 3 & 5 & 3 \\
\hline Portugal & 7 & -1 & -13 & 13 & 12 & 30 & 28 & 4 & 2 & 14 & 8 & 3 \\
\hline Romania & 6 & 9 & 14 & 10 & 28 & 26 & 10 & 5 & 3 & 12 & 7 & 8 \\
\hline Moldova & 7 & 4 & 16 & 12 & 12 & 26 & 14 & 6 & 6 & 16 & 11 & 9 \\
\hline Slovenia & 17 & 8 & 15 & 12 & 15 & 25 & 9 & 8 & 1 & 23 & 12 & 8 \\
\hline Spain & 6 & -4 & -11 & 10 & 15 & 24 & 33 & 4 & 1 & 13 & 8 & 3 \\
\hline Sweden & 3 & 8 & 19 & 8 & 24 & 18 & 44 & 3 & 1 & 4 & 4 & 2 \\
\hline Switzerland & 6 & 9 & 13 & 8 & 14 & 41 & 13 & 4 & 2 & 6 & 14 & 6 \\
\hline Tunisia & -9 & -15 & -23 & 14 & 14 & 45 & 14 & 3 & 5 & 5 & 9 & 5 \\
\hline Turkey & -1 & -5 & -5 & 10 & 11 & 69 & 3 & 3 & 0 & 3 & 6 & 5 \\
\hline UK & 2 & 7 & 13 & 8 & 39 & 18 & 21 & 3 & 2 & 9 & 5 & 2 \\
\hline Ukraine & 5 & 7 & 15 & 9 & 13 & 42 & 18 & 5 & 1 & 9 & 8 & 5 \\
\hline
\end{tabular}


Table S6. Absolute changes of mean temperature in summer (JJA) in the near future (2021-2050) compared to the period 1981-2010 (see legend of Table S2 for further details).

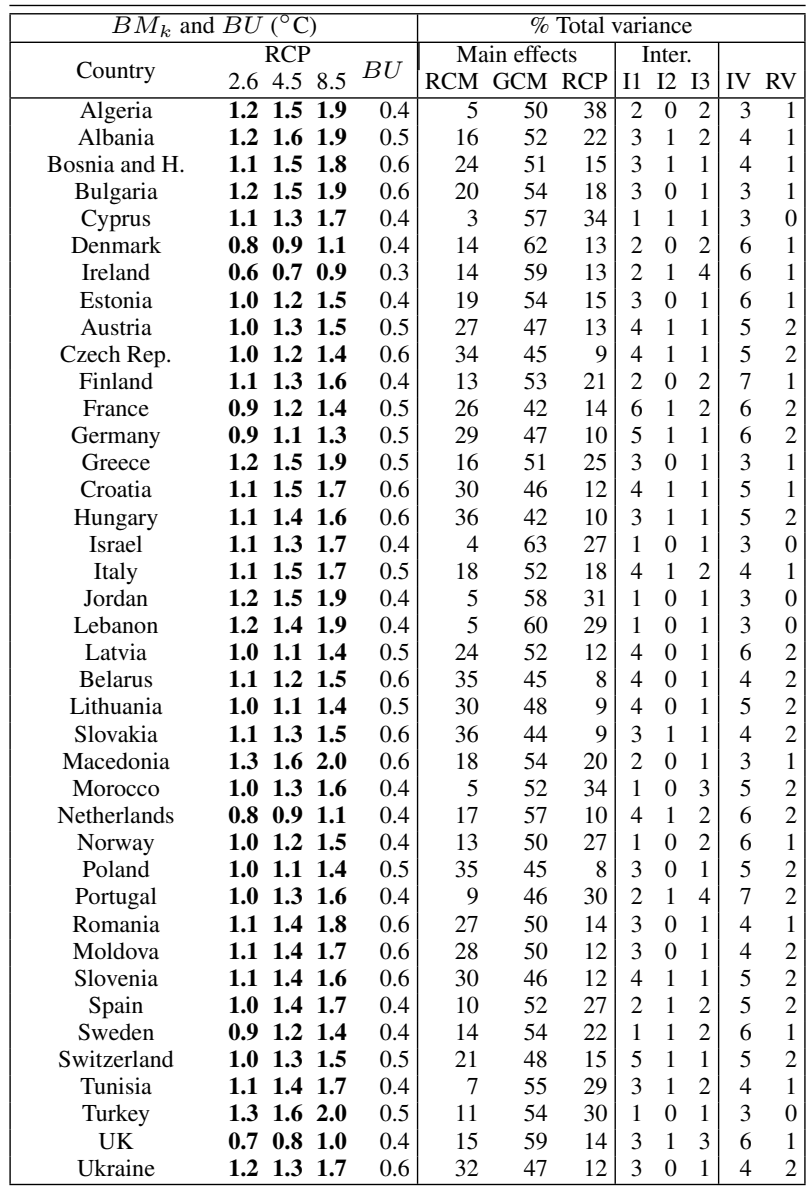


Table S7. Absolute changes of mean temperature in summer (JJA) at the end of the century (2071-2099) compared to the period 1981-2010 (see legend of Table S2 for further details).

\begin{tabular}{|c|c|c|c|c|c|c|c|c|c|c|}
\hline \multicolumn{3}{|c|}{$B M_{k}$ and $B U\left({ }^{\circ} \mathrm{C}\right)$} & \multicolumn{8}{|c|}{$\%$ Total variance } \\
\hline \multirow{2}{*}{ Country } & $\mathrm{RCP}$ & \multirow{2}{*}{$B U$} & \multicolumn{3}{|c|}{ Main effects } & \multicolumn{3}{|c|}{ Inter. } & \multirow[b]{2}{*}{ IV } & \\
\hline & $2.6 \quad 4.5 \quad 8.5$ & & $\mathrm{RCM}$ & GCM & $\mathrm{RCP}$ & $\mathrm{I} 1$ & & $\mathrm{I} 3$ & & RV \\
\hline Algeria & $\begin{array}{lll}1.7 & 2.9 & 5.4\end{array}$ & 0.8 & 2 & 18 & 77 & 0 & 0 & 1 & 0 & 0 \\
\hline Albania & $\begin{array}{lll}1.6 & 3.0 & 5.2\end{array}$ & 1.1 & 11 & 20 & 64 & 2 & 0 & 1 & 0 & 1 \\
\hline Bosnia and $\mathrm{H}$. & $\begin{array}{lll}1.6 & 2.8 & 4.9\end{array}$ & 1.3 & 18 & 24 & 52 & 2 & 1 & 1 & 0 & 1 \\
\hline Bulgaria & $\begin{array}{lll}1.7 & 3.0 & 5.2\end{array}$ & 1.2 & 12 & 25 & 60 & 1 & 0 & 1 & 0 & 1 \\
\hline Cyprus & $\begin{array}{lll}1.4 & 2.5 & 4.5\end{array}$ & 0.7 & 1 & 18 & 79 & 0 & 0 & 1 & 0 & 0 \\
\hline Denmark & $\begin{array}{lll}1.1 & 1.8 & 3.0\end{array}$ & 0.7 & 9 & 33 & 53 & 1 & 0 & 2 & 1 & 1 \\
\hline Ireland & $\begin{array}{lll}0.7 & 1.4 & 2.7\end{array}$ & 0.7 & 6 & 31 & 59 & 1 & 0 & 1 & 1 & 1 \\
\hline Estonia & $\begin{array}{lll}1.5 & 2.4 & 3.8\end{array}$ & 1.0 & 18 & 26 & 49 & 1 & 1 & 2 & 1 & 1 \\
\hline Austria & $\begin{array}{lll}1.5 & 2.5 & 4.3\end{array}$ & 1.2 & 20 & 23 & 49 & 3 & 1 & 1 & 1 & 2 \\
\hline Czech Rep. & $\begin{array}{lll}1.4 & 2.3 & 3.9\end{array}$ & 1.2 & 26 & 25 & 43 & 3 & 1 & 1 & 1 & 2 \\
\hline Finland & $\begin{array}{lll}1.6 & 2.6 & 4.1\end{array}$ & 0.9 & 11 & 26 & 58 & 1 & 0 & 2 & 1 & 1 \\
\hline France & $\begin{array}{lll}1.1 & 2.2 & 4.1\end{array}$ & 1.0 & 17 & 17 & 58 & 3 & 1 & 2 & 1 & 1 \\
\hline Germany & 1.22 .13 .6 & 1.0 & 22 & 24 & 47 & 3 & 1 & 1 & 1 & 1 \\
\hline Greece & $\begin{array}{lll}1.7 & 3.0 & 5.2\end{array}$ & 1.0 & 9 & 19 & 68 & 1 & 0 & 1 & 0 & 1 \\
\hline Croatia & $\begin{array}{lll}1.5 & 2.7 & 4.8\end{array}$ & 1.3 & 21 & 22 & 51 & 3 & 1 & 1 & 1 & 1 \\
\hline Hungary & $\begin{array}{lll}1.5 & 2.7 & 4.5\end{array}$ & 1.3 & 23 & 22 & 48 & 3 & 1 & 2 & 1 & 2 \\
\hline Israel & $\begin{array}{lll}1.5 & 2.6 & 4.5\end{array}$ & 0.8 & 2 & 24 & 71 & 1 & 0 & 1 & 0 & 0 \\
\hline Italy & $\begin{array}{lll}1.4 & 2.7 & 4.9\end{array}$ & 1.1 & 12 & 21 & 60 & 2 & 1 & 1 & 0 & 1 \\
\hline Jordan & $\begin{array}{lll}1.6 & 2.9 & 5.2\end{array}$ & 0.8 & 3 & 19 & 76 & 0 & 0 & 1 & 0 & 0 \\
\hline Lebanon & $\begin{array}{lll}1.6 & 2.8 & 4.9\end{array}$ & 0.8 & 2 & 20 & 75 & 0 & 0 & 1 & 0 & 0 \\
\hline Latvia & $\begin{array}{lll}1.5 & 2.3 & 3.8\end{array}$ & 1.0 & 23 & 25 & 45 & 2 & 1 & 2 & 1 & 2 \\
\hline Belarus & $\begin{array}{lll}1.6 & 2.5 & 4.1\end{array}$ & 1.2 & 31 & 21 & 40 & 2 & 1 & 2 & 1 & 2 \\
\hline Lithuania & $\begin{array}{lll}1.5 & 2.3 & 3.8\end{array}$ & 1.1 & 27 & 24 & 42 & 2 & 1 & 2 & 1 & 2 \\
\hline Slovakia & $\begin{array}{lll}1.5 & 2.6 & 4.3\end{array}$ & 1.3 & 27 & 22 & 44 & 2 & 1 & 1 & 1 & 2 \\
\hline Macedonia & $\begin{array}{lll}1.7 & 3.1 & 5.4\end{array}$ & 1.2 & 11 & 23 & 62 & 1 & 0 & 1 & 0 & 1 \\
\hline Morocco & $\begin{array}{lll}1.3 & 2.6 & 4.6\end{array}$ & 0.6 & 2 & 14 & 82 & 0 & 0 & 1 & 0 & 1 \\
\hline Netherlands & $\begin{array}{lll}1.0 & 1.8 & 3.1\end{array}$ & 0.8 & 12 & 28 & 53 & 2 & 1 & 1 & 1 & 1 \\
\hline Norway & $\begin{array}{lll}1.4 & 2.4 & 3.9\end{array}$ & 0.7 & 8 & 23 & 65 & 0 & 0 & 1 & 1 & 1 \\
\hline Poland & $\begin{array}{lll}1.4 & 2.3 & 3.8\end{array}$ & 1.2 & 28 & 25 & 41 & 2 & 0 & 2 & 1 & 2 \\
\hline Portugal & $\begin{array}{lll}1.1 & 2.5 & 4.4\end{array}$ & 0.7 & 5 & 10 & 81 & 1 & 0 & 1 & 1 & 1 \\
\hline Romania & $\begin{array}{lll}1.7 & 2.9 & 4.9\end{array}$ & 1.3 & 20 & 23 & 52 & 2 & 1 & 2 & 0 & 1 \\
\hline Moldova & $\begin{array}{lll}1.8 & 2.8 & 4.8\end{array}$ & 1.3 & 23 & 23 & 48 & 2 & 1 & 2 & 1 & 1 \\
\hline Slovenia & $\begin{array}{lll}1.5 & 2.6 & 4.6\end{array}$ & 1.4 & 23 & 24 & 46 & 3 & 1 & 1 & 1 & 2 \\
\hline Spain & $\begin{array}{lll}1.3 & 2.7 & 4.8\end{array}$ & 0.8 & 6 & 15 & 75 & 1 & 0 & 1 & 0 & 1 \\
\hline Sweden & $\begin{array}{lll}1.4 & 2.3 & 3.7\end{array}$ & 0.8 & 12 & 24 & 61 & 1 & 0 & 1 & 1 & 1 \\
\hline Switzerland & $\begin{array}{lll}1.4 & 2.5 & 4.5\end{array}$ & 1.2 & 16 & 22 & 55 & 3 & 1 & 1 & 1 & 2 \\
\hline Tunisia & $\begin{array}{lll}1.5 & 2.7 & 4.9\end{array}$ & 0.8 & 3 & 20 & 73 & 1 & 0 & 1 & 0 & 1 \\
\hline Turkey & $\begin{array}{lll}1.8 & 3.1 & 5.4\end{array}$ & 1.0 & 5 & 23 & 70 & 0 & 0 & 1 & 0 & 0 \\
\hline UK & $\begin{array}{lll}0.8 & 1.6 & 2.9\end{array}$ & 0.7 & 9 & 27 & 59 & 1 & 0 & 1 & 1 & 1 \\
\hline Ukraine & $\begin{array}{llll}1.8 & 2.8 & 4.7\end{array}$ & 1.2 & 24 & 22 & 48 & 1 & 1 & 2 & 1 & 1 \\
\hline
\end{tabular}


Table S8. Relative changes of total precipitation in summer (JJA) in the near future (2021-2050) compared to the period 1981-2010 (see legend of Table S2 for further details).

\begin{tabular}{|c|c|c|c|c|c|c|c|c|c|c|c|c|}
\hline \multicolumn{5}{|c|}{$B M_{k}$ and $B U(\%)$} & \multicolumn{8}{|c|}{$\%$ Total variance } \\
\hline \multirow{2}{*}{ Country } & \multicolumn{3}{|c|}{$\mathrm{RCP}$} & \multirow[b]{2}{*}{$B U$} & \multicolumn{3}{|c|}{ Main effects } & \multicolumn{3}{|c|}{ Inter. } & \multirow[b]{2}{*}{ IV } & \multirow[b]{2}{*}{ RV } \\
\hline & 2.6 & 4.5 & 8.5 & & $\mathrm{RCM}$ & GCM & $\mathrm{RCP}$ & I1 & $\mathrm{I} 2$ & I3 & & \\
\hline Algeria & -5 & -10 & -8 & 17 & 17 & 57 & 2 & 3 & 0 & 2 & 15 & 4 \\
\hline Albania & -4 & -10 & -13 & 11 & 13 & 39 & 11 & 8 & 2 & 7 & 14 & 5 \\
\hline Bosnia and $\mathrm{H}$. & -3 & -7 & -8 & 9 & 17 & 39 & 5 & 10 & 2 & 6 & 16 & 5 \\
\hline Bulgaria & -3 & -8 & -11 & 9 & 9 & 39 & 12 & 9 & 1 & 6 & 20 & 6 \\
\hline Cyprus & -8 & -19 & -16 & 21 & 4 & 41 & 5 & 8 & 1 & 3 & 33 & 5 \\
\hline Denmark & -1 & 0 & -1 & 7 & 41 & 24 & 0 & 5 & 1 & 4 & 19 & 5 \\
\hline Ireland & -4 & -5 & -7 & 4 & 25 & 20 & 8 & 5 & 2 & 10 & 27 & 4 \\
\hline Estonia & -1 & 2 & 4 & 7 & 19 & 36 & 6 & 7 & 1 & 4 & 19 & 8 \\
\hline Austria & 0 & -1 & 0 & 6 & 28 & 31 & 1 & 14 & 2 & 2 & 16 & 6 \\
\hline Czech Rep. & 0 & 0 & 1 & 6 & 25 & 28 & 1 & 13 & 1 & 2 & 22 & 8 \\
\hline Finland & 2 & 4 & 5 & 5 & 18 & 29 & 9 & 7 & 1 & 5 & 24 & 6 \\
\hline France & -3 & -6 & -8 & 7 & 25 & 34 & 9 & 9 & 2 & 4 & 13 & 4 \\
\hline Germany & -1 & -1 & -1 & 6 & 35 & 27 & 0 & 11 & 1 & 3 & 15 & 8 \\
\hline Greece & -4 & -9 & -14 & 11 & 10 & 35 & 13 & 10 & 1 & 8 & 17 & 5 \\
\hline Croatia & -2 & -6 & -6 & 9 & 24 & 31 & 4 & 10 & 2 & 5 & 18 & 5 \\
\hline Hungary & -1 & -4 & -3 & 7 & 21 & 27 & 4 & 9 & 2 & 4 & 27 & 7 \\
\hline Israel & -12 & -22 & -18 & 28 & 7 & 21 & 2 & 16 & 1 & 7 & 39 & 7 \\
\hline Italy & -0 & -5 & -6 & 8 & 19 & 39 & 9 & 9 & 2 & 6 & 12 & 3 \\
\hline Jordan & -14 & -8 & -6 & 37 & 19 & 25 & 1 & 6 & 1 & 3 & 42 & 3 \\
\hline Lebanon & -8 & -18 & -17 & 22 & 8 & 29 & 4 & 9 & 2 & 4 & 38 & 6 \\
\hline Latvia & -0 & 2 & 3 & 7 & 23 & 33 & 3 & 10 & 1 & 4 & 19 & 7 \\
\hline Belarus & -2 & 0 & 0 & 8 & 16 & 43 & 1 & 13 & 1 & 3 & 17 & 6 \\
\hline Lithuania & -1 & 1 & 2 & 8 & 23 & 34 & 2 & 11 & 1 & 3 & 19 & 6 \\
\hline Slovakia & -1 & -3 & -2 & 7 & 17 & 36 & 2 & 11 & 2 & 2 & 23 & 7 \\
\hline Macedonia & -4 & -9 & -12 & 9 & 12 & 38 & 12 & 6 & 1 & 7 & 19 & 5 \\
\hline Morocco & -7 & -10 & -12 & 18 & 9 & 73 & 1 & 2 & 0 & 2 & 11 & 1 \\
\hline Netherlands & -3 & -3 & -3 & 7 & 36 & 23 & 0 & 10 & 2 & 3 & 18 & 8 \\
\hline Norway & 2 & 3 & 4 & 4 & 35 & 29 & 6 & 3 & 1 & 3 & 19 & 4 \\
\hline Poland & -0 & 0 & 1 & 7 & 29 & 33 & 1 & 11 & 1 & 2 & 16 & 8 \\
\hline Portugal & -6 & -14 & -18 & 10 & 15 & 29 & 21 & 3 & 2 & 5 & 21 & 3 \\
\hline Romania & -1 & -5 & -6 & 7 & 11 & 37 & 7 & 11 & 2 & 4 & 22 & 7 \\
\hline Moldova & 0 & -3 & -4 & 8 & 17 & 32 & 5 & 8 & 2 & 2 & 29 & 4 \\
\hline Slovenia & -2 & -5 & -5 & 8 & 26 & 30 & 4 & 9 & 2 & 3 & 20 & 6 \\
\hline Spain & -3 & -9 & -12 & 9 & 14 & 51 & 15 & 2 & 1 & 3 & 12 & 3 \\
\hline Sweden & 2 & 4 & 5 & 5 & 21 & 42 & 5 & 5 & 1 & 5 & 16 & 4 \\
\hline Switzerland & -1 & -2 & -3 & 7 & 26 & 37 & 2 & 14 & 2 & 3 & 11 & 4 \\
\hline Tunisia & -5 & -13 & -15 & 17 & 13 & 44 & 6 & 10 & 1 & 5 & 17 & 4 \\
\hline Turkey & -5 & -12 & -14 & 9 & 14 & 39 & 14 & 6 & 1 & 3 & 20 & 3 \\
\hline UK & -2 & -3 & -5 & 5 & 37 & 19 & 4 & 9 & 1 & 7 & 20 & 4 \\
\hline Ukraine & -2 & -4 & -4 & 8 & 15 & 47 & 2 & 9 & 1 & 3 & 19 & 4 \\
\hline
\end{tabular}


Table S9. Relative changes of total precipitation in summer (JJA) at the end of the century (2071-2099) compared to the period 1981-2010 (see legend of Table S2 for further details).

\begin{tabular}{|c|c|c|c|c|c|c|c|c|c|c|c|c|}
\hline \multicolumn{5}{|c|}{$B M_{k}$ and $B U(\%)$} & \multicolumn{8}{|c|}{$\%$ Total variance } \\
\hline \multirow{2}{*}{ Country } & \multicolumn{3}{|c|}{$\mathrm{RCP}$} & \multirow{2}{*}{$B U$} & \multicolumn{3}{|c|}{ Main effects } & \multicolumn{3}{|c|}{ Inter. } & \multirow[b]{2}{*}{ IV } & \multirow[b]{2}{*}{ RV } \\
\hline & 2.6 & 4.5 & 8.5 & & $\mathrm{RCM}$ & GCM & $\mathrm{RCP}$ & & $\mathrm{I} 2$ & I3 & & \\
\hline Algeria & -14 & -20 & -22 & 33 & 14 & 68 & 1 & 4 & 1 & 4 & 4 & 5 \\
\hline Albania & -4 & -18 & -30 & 20 & 18 & 23 & 23 & 11 & 3 & 9 & 4 & 8 \\
\hline Bosnia and $\mathrm{H}$. & -3 & -13 & -20 & 17 & 22 & 32 & 13 & 10 & 2 & 8 & 4 & 8 \\
\hline Bulgaria & -4 & -16 & -26 & 16 & 12 & 38 & 23 & 8 & 1 & 6 & 5 & 8 \\
\hline Cyprus & -14 & -31 & -39 & 38 & 4 & 42 & 7 & 11 & 5 & 7 & 10 & 14 \\
\hline Denmark & -3 & 0 & -4 & 15 & 46 & 30 & 1 & 5 & 2 & 5 & 4 & 7 \\
\hline Ireland & -7 & -11 & -18 & 9 & 24 & 21 & 20 & 7 & 3 & 14 & 6 & 5 \\
\hline Estonia & -2 & 4 & 8 & 15 & 24 & 40 & 6 & 8 & 1 & 6 & 4 & 11 \\
\hline Austria & 2 & -1 & -2 & 13 & 29 & 39 & 1 & 13 & 3 & 3 & 3 & 7 \\
\hline Czech Rep. & 1 & 0 & 0 & 14 & 25 & 41 & 0 & 13 & 2 & 4 & 5 & 9 \\
\hline Finland & 2 & 7 & 12 & 11 & 23 & 31 & 11 & 8 & 2 & 11 & 5 & 9 \\
\hline France & -5 & -14 & -23 & 15 & 27 & 35 & 18 & 7 & 2 & 5 & 2 & 4 \\
\hline Germany & -2 & -3 & -5 & 15 & 38 & 37 & 1 & 9 & 2 & 4 & 3 & 8 \\
\hline Greece & -6 & -18 & -34 & 19 & 11 & 29 & 27 & 9 & 2 & 10 & 5 & 7 \\
\hline Croatia & -1 & -9 & -16 & 17 & 32 & 26 & 11 & 10 & 2 & 8 & 4 & 7 \\
\hline Hungary & 1 & -7 & -8 & 15 & 24 & 28 & 8 & 13 & 2 & 9 & 6 & 10 \\
\hline Israel & -14 & -19 & -37 & 41 & 5 & 30 & 5 & 19 & 2 & 13 & 17 & 9 \\
\hline Italy & 2 & -10 & -17 & 17 & 25 & 33 & 16 & 10 & 2 & 7 & 3 & 5 \\
\hline Jordan & -21 & 10 & -3 & 71 & 26 & 38 & 3 & 11 & 2 & 3 & 11 & 6 \\
\hline Lebanon & -22 & -25 & -43 & 36 & 8 & 39 & 6 & 13 & 5 & 8 & 14 & 8 \\
\hline Latvia & -2 & 3 & 5 & 16 & 28 & 42 & 3 & 9 & 1 & 5 & 4 & 7 \\
\hline Belarus & -4 & -3 & -2 & 18 & 24 & 49 & 0 & 11 & 1 & 4 & 4 & 6 \\
\hline Lithuania & -2 & 2 & 3 & 17 & 31 & 43 & 2 & 9 & 1 & 4 & 4 & 6 \\
\hline Slovakia & 0 & -5 & -7 & 15 & 19 & 41 & 4 & 14 & 2 & 6 & 5 & 9 \\
\hline Macedonia & -7 & -18 & -29 & 16 & 13 & 33 & 24 & 6 & 2 & 9 & 5 & 8 \\
\hline Morocco & -13 & -23 & -30 & 33 & 11 & 74 & 4 & 2 & 0 & 4 & 3 & 2 \\
\hline Netherlands & -3 & -5 & -9 & 16 & 42 & 29 & 2 & 7 & 2 & 4 & 4 & 10 \\
\hline Norway & 2 & 6 & 9 & 9 & 40 & 29 & 11 & 3 & 2 & 4 & 3 & 6 \\
\hline Poland & 0 & -1 & 1 & 16 & 35 & 39 & 0 & 12 & 1 & 3 & 3 & 8 \\
\hline Portugal & -11 & -31 & -46 & 17 & 13 & 31 & 42 & 1 & 2 & 2 & 5 & 4 \\
\hline Romania & -2 & -10 & -15 & 14 & 15 & 39 & 13 & 11 & 2 & 4 & 5 & 10 \\
\hline Moldova & -1 & -6 & -11 & 16 & 18 & 41 & 7 & 11 & 4 & 4 & 8 & 8 \\
\hline Slovenia & -1 & -7 & -14 & 16 & 35 & 27 & 9 & 10 & 2 & 5 & 4 & 7 \\
\hline Spain & -7 & -20 & -34 & 18 & 14 & 46 & 28 & 2 & 1 & 4 & 3 & 3 \\
\hline Sweden & 3 & 7 & 10 & 11 & 28 & 43 & 7 & 5 & 1 & 7 & 3 & 6 \\
\hline Switzerland & -0 & -5 & -9 & 16 & 30 & 41 & 5 & 11 & 2 & 4 & 2 & 5 \\
\hline Tunisia & -10 & -26 & -40 & 30 & 20 & 40 & 15 & 8 & 1 & 6 & 5 & 5 \\
\hline Turkey & -11 & -25 & -35 & 17 & 12 & 42 & 25 & 5 & 2 & 5 & 5 & 4 \\
\hline UK & -4 & -6 & -12 & 10 & 40 & 22 & 12 & 7 & 2 & 9 & 4 & 4 \\
\hline Ukraine & -3 & -9 & -12 & 15 & 23 & 50 & 5 & 8 & 2 & 4 & 5 & 4 \\
\hline
\end{tabular}




\section{Main results for the capitals of the domain}

Table S10. Mean expected change response $B M_{k}$, scenario-excluded uncertainty $B U$ and partition of the total uncertainty for the capital cities of the domain, for absolute changes of mean temperature in winter (DJF) in the near future (2021-2050) compared to the period 1981-2010. I1, I2 and I3 refer to interactions RCM/GCM, RCP/RCM and RCP/GCM, respectively. IV and RV refer to internal and residual variability, respectively. Absolute mean expected changes greater than the uncertainty $(|B U / B M|>1)$ are indicated in bold font.

\begin{tabular}{|c|c|c|c|c|c|c|c|c|c|c|}
\hline \multirow[b]{2}{*}{ Capital city } & \multirow[b]{2}{*}{ Country } & \multicolumn{2}{|c|}{$B M_{k} \& B U\left({ }^{\circ} \mathrm{C}\right)$} & \multicolumn{7}{|c|}{$\%$ Total variance } \\
\hline & & \begin{tabular}{ccc}
\multicolumn{2}{c}{$\mathrm{RCP}$} \\
2.6 & 4.5 & 8.5
\end{tabular} & $B U$ & $\begin{array}{r}\mathrm{M} \\
\mathrm{RCM}\end{array}$ & $\begin{array}{r}\text { ain effe } \\
\text { GCM }\end{array}$ & $\begin{array}{l}\text { cts } \\
\mathrm{RCP}\end{array}$ & In $\mathrm{I}$ & $\begin{array}{ll}\text { Iter. } \\
\text { I2 I3 }\end{array}$ & IV & RV \\
\hline Amman & Jordan & $\begin{array}{|lll|}0.9 & 1.0 & 1.3\end{array}$ & 0.4 & 7 & 60 & 19 & 1 & 14 & 7 & 1 \\
\hline Algiers & Algeria & $\begin{array}{lll}0.7 & 0.8 & 1.1\end{array}$ & 0.3 & 2 & 58 & 29 & 2 & 0 & 6 & 1 \\
\hline Amsterdam & Netherlands & $\begin{array}{llll}0.6 & 0.8 & 1.1\end{array}$ & 0.3 & 4 & 44 & 27 & 5 & 1 & 10 & 3 \\
\hline Andorra la Vella & Andorra & $\begin{array}{lll}0.8 & 1.1 & 1.4\end{array}$ & 0.4 & 21 & 32 & 32 & 4 & 1 & 6 & 2 \\
\hline Ankara & Turkey & $\begin{array}{lll}1.0 & 1.3 & 1.5\end{array}$ & 0.5 & 33 & 33 & 14 & 4 & 1 & 9 & 2 \\
\hline Athens & Greece & $\begin{array}{llll}0.8 & 0.9 & 1.2\end{array}$ & 0.3 & 5 & 55 & 21 & 1 & 1 & 9 & 1 \\
\hline Bayrut & Lebanon & $\begin{array}{lll}0.8 & 0.9 & 1.2\end{array}$ & 0.3 & 2 & 56 & 25 & 1 & 1 & 8 & 1 \\
\hline Belgrade & Serbia and $\mathrm{M}$. & $\begin{array}{lll}0.9 & 1.3 & 1.5\end{array}$ & 0.4 & 22 & 31 & 20 & 4 & 1 & 12 & 3 \\
\hline Berlin & Germany & $\begin{array}{lll}0.7 & 1.0 & 1.3\end{array}$ & 0.4 & 6 & 41 & 24 & 7 & 1 & 12 & 3 \\
\hline Bern & Switzerland & $\begin{array}{lll}0.8 & 1.1 & 1.3\end{array}$ & 0.4 & 8 & 41 & 26 & 6 & 1 & 10 & 3 \\
\hline Bratislava & Slovakia & $\begin{array}{lll}0.9 & 1.2 & 1.5\end{array}$ & 0.5 & 17 & 32 & 23 & 7 & 1 & 11 & 3 \\
\hline Brussels & Belgium & $\begin{array}{lll}0.6 & 0.9 & 1.1\end{array}$ & 0.3 & 7 & 32 & 30 & 7 & 1 & 11 & 3 \\
\hline Bucharest & Romania & $\begin{array}{lll}1.0 & 1.3 & 1.6\end{array}$ & 0.5 & 26 & 28 & 18 & 4 & 1 & 13 & 5 \\
\hline Budapest & Hungary & $\begin{array}{lll}0.9 & 1.2 & 1.5\end{array}$ & 0.4 & 22 & 27 & 24 & 6 & 1 & 12 & 3 \\
\hline Cairo & Egypt & $\begin{array}{llll}0.8 & 0.9 & 1.2\end{array}$ & 0.3 & 14 & 45 & 27 & 1 & 1 & 6 & 1 \\
\hline Chisinau & Moldova & $\begin{array}{lll}1.0 & 1.4 & 1.6\end{array}$ & 0.4 & 20 & 24 & 22 & 5 & 2 & 18 & 4 \\
\hline Copenhagen & Denmark & $\begin{array}{lll}0.7 & 1.0 & 1.2\end{array}$ & 0.4 & 7 & 45 & 26 & 4 & 1 & 10 & 2 \\
\hline Damascus & Syria & $\begin{array}{lll}0.9 & 1.1 & 1.4\end{array}$ & 0.3 & 7 & 57 & 22 & 1 & 1 & 7 & 1 \\
\hline Douglas & Isle of Man & $\begin{array}{lll}0.4 & 0.6 & 0.8\end{array}$ & 0.3 & 10 & 47 & 29 & 3 & 0 & 8 & 1 \\
\hline Dublin & Ireland & $\begin{array}{lll}0.4 & 0.6 & 0.8\end{array}$ & 0.3 & 7 & 49 & 30 & 2 & 0 & 9 & 1 \\
\hline Gibraltar & Gibraltar & $\begin{array}{lll}0.6 & 0.7 & 1.0\end{array}$ & 0.2 & 6 & 49 & 30 & 2 & 1 & 6 & 3 \\
\hline Helsi & Finland & $\begin{array}{lll}1.2 & 1.5 & 1.9\end{array}$ & 0.7 & 13 & 46 & 14 & 9 & 1 & 10 & 3 \\
\hline Jerusalem & Israel & $\begin{array}{lll}0.9 & 1.0 & 1.3\end{array}$ & 0.3 & 4 & 58 & 24 & 1 & 1 & 8 & 1 \\
\hline Kiev & Ukraine & $\begin{array}{lll}1.0 & 1.3 & 1.6\end{array}$ & 0.5 & 15 & 30 & 18 & 7 & 2 & 19 & 3 \\
\hline Lisbon & Portugal & $\begin{array}{lll}0.6 & 0.8 & 0.9\end{array}$ & 0.2 & 7 & 47 & 30 & 2 & 1 & 8 & 1 \\
\hline Ljubljana & Slovenia & $\begin{array}{lll}0.9 & 1.2 & 1.4\end{array}$ & 0.4 & 13 & 36 & 24 & 6 & 1 & 11 & 3 \\
\hline London & UK & $\begin{array}{lll}0.5 & 0.7 & 0.9\end{array}$ & 0.3 & 8 & 42 & 29 & 5 & 1 & 9 & 2 \\
\hline Luxemburg & Luxembourg & $\begin{array}{lll}0.6 & 0.9 & 1.1\end{array}$ & 0.3 & 6 & 38 & 27 & 7 & 1 & 10 & 3 \\
\hline Madrid & Spain & $\begin{array}{lll}0.7 & 0.9 & 1.1\end{array}$ & 0.3 & 11 & 43 & 33 & 2 & 1 & 7 & 1 \\
\hline Minsk & Bel & $\begin{array}{lll}1.0 & 1.3 & 1.6\end{array}$ & 0.5 & 10 & 34 & 20 & 9 & 2 & 17 & 4 \\
\hline Monace & Mo & $\begin{array}{lll}0.7 & 0.9 & 1.2\end{array}$ & 0.3 & 6 & 51 & 30 & 2 & 0 & 6 & 2 \\
\hline $\mathrm{Nicc}$ & & $\begin{array}{lll}0.8 & 1.0 & 1.2\end{array}$ & 0.3 & 5 & 50 & 29 & 1 & 1 & 8 & 1 \\
\hline slo & Nor & $\begin{array}{lll}0.8 & 1.2 & 1.5\end{array}$ & 0.5 & 13 & 44 & 21 & 5 & 1 & 10 & 2 \\
\hline Paris & France & $\begin{array}{lll}0.6 & 0.8 & 1.1\end{array}$ & 0.3 & 9 & 36 & 27 & 5 & 1 & 10 & 3 \\
\hline Prague & Czech Republic & $\begin{array}{lll}0.8 & 1.1 & 1.4\end{array}$ & 0.4 & 12 & 36 & 23 & 6 & 1 & 12 & 3 \\
\hline Rabat & Morocco & $\begin{array}{lll}0.6 & 0.8 & 1.0\end{array}$ & 0.2 & 7 & 39 & 33 & 2 & 1 & 9 & 3 \\
\hline Riga & Latvia & $\begin{array}{lll}1.0 & 1.3 & 1.6\end{array}$ & 0.6 & 12 & 37 & 17 & 9 & 1 & 15 & 3 \\
\hline Rome & Italy & $\begin{array}{lll}0.8 & 1.0 & 1.2\end{array}$ & 0.3 & 12 & 50 & 23 & 1 & 0 & 8 & 2 \\
\hline Saint Helier & Jersey & $\begin{array}{lll}0.5 & 0.7 & 0.9\end{array}$ & 0.3 & 3 & 61 & 23 & 2 & 0 & 6 & 1 \\
\hline Saint Peter Port & Guernsey and A. & $\begin{array}{lll}0.5 & 0.6 & 0.8\end{array}$ & 0.3 & 3 & 62 & 23 & 2 & 0 & 6 & 1 \\
\hline San Marino & San Marino & $\begin{array}{lll}0.8 & 1.0 & 1.3\end{array}$ & 0.4 & 10 & 47 & 23 & 3 & 1 & 9 & 2 \\
\hline Sarajevo & Bosnia a & $\begin{array}{lll}0.9 & 1.2 & 1.5\end{array}$ & 0.5 & 22 & 35 & 19 & 3 & 1 & 10 & 3 \\
\hline Skor & $\mathrm{Ma}$ & $\begin{array}{llll}0.9 & 1.2 & 1.4\end{array}$ & 0.4 & 19 & 35 & 21 & 3 & 1 & 10 & 3 \\
\hline Sofia & Bulgaria & $\begin{array}{lll}0.9 & 1.2 & 1.4\end{array}$ & 0.4 & 19 & 31 & 22 & 2 & 0 & 13 & 4 \\
\hline Stockholm & Sweden & $\begin{array}{lll}0.9 & 1.2 & 1.6\end{array}$ & 0.5 & 16 & 36 & 22 & 8 & 1 & 10 & 3 \\
\hline Tallinn & Estonia & $\begin{array}{lll}1.1 & 1.3 & 1.7\end{array}$ & 0.6 & 14 & 44 & 14 & 9 & 1 & 12 & 3 \\
\hline Tirana & Albania & $\begin{array}{lll}0.8 & 1.0 & 1.2\end{array}$ & 0.3 & 11 & 48 & 22 & 1 & 1 & 9 & 2 \\
\hline Torshavn & Faroe Islands & $\begin{array}{lll}0.4 & \mathbf{0 . 6} & \mathbf{0 . 8}\end{array}$ & 0.5 & 4 & 80 & 11 & 0 & 0 & 3 & 1 \\
\hline Tripoli & Libya & $\begin{array}{lll}0.8 & 0.9 & 1.2\end{array}$ & 0.3 & 10 & 44 & 30 & 2 & 1 & 7 & 1 \\
\hline Tunis & Tunisia & $\begin{array}{lll}0.8 & 0.9 & 1.2\end{array}$ & 0.3 & 8 & 51 & 29 & 1 & 0 & 7 & 1 \\
\hline Vaduz & Liechtenstein & $\begin{array}{lll}0.8 & 1.1 & 1.3\end{array}$ & 0.4 & 15 & 42 & 22 & 6 & 1 & 9 & 2 \\
\hline Valletta & Malta & $\begin{array}{lll}0.7 & 0.9 & 1.1\end{array}$ & 0.3 & 3 & 57 & 28 & 1 & 0 & 7 & 1 \\
\hline Vatican City & Vatican City & $\begin{array}{lll}0.8 & 1.0 & 1.2\end{array}$ & 0.3 & 12 & 50 & 23 & 1 & 0 & 8 & 2 \\
\hline Vienna & Austria & $\begin{array}{lll}0.8 & 1.2 & 1.4\end{array}$ & 0.4 & 16 & 34 & 23 & 6 & 1 & 11 & 3 \\
\hline Vilnius & Lithuania & $\begin{array}{lll}1.0 & 1.3 & 1.6\end{array}$ & 0.5 & 9 & 34 & 21 & 8 & 2 & 17 & 4 \\
\hline Warsaw & Poland & $\begin{array}{lll}0.9 & 1.2 & 1.5\end{array}$ & 0.5 & 10 & 37 & 20 & 9 & 2 & 13 & 4 \\
\hline Zagreb & Croatia & $\begin{array}{lll}0.9 & 1.2 & 1.5\end{array}$ & 0.4 & 18 & 29 & 23 & 8 & 1 & 11 & 4 \\
\hline
\end{tabular}


Table S11. Absolute changes of mean temperature in winter (DJF) at the end of the century (2071-2099) compared to the period 1981-2010 (see legend of Table S10 for further details).

\begin{tabular}{|c|c|c|c|c|c|c|c|c|c|c|c|}
\hline \multirow[b]{2}{*}{ Capital city } & \multirow[b]{2}{*}{ Country } & \multicolumn{2}{|c|}{$B M_{k} \& B U\left({ }^{\circ} \mathrm{C}\right)$} & \multicolumn{8}{|c|}{$\%$ Total variance } \\
\hline & & \begin{tabular}{ccc}
\multicolumn{3}{c}{$\mathrm{RCP}$} \\
2.6 & 4.5 & 8.5
\end{tabular} & $B U$ & $\begin{array}{r}\mathrm{M} \\
\mathrm{RCM}\end{array}$ & $\begin{array}{r}\text { in effe } \\
\text { GCM }\end{array}$ & RCP & & & I3 & IV & RV \\
\hline Amman & Jordan & $\begin{array}{lll}1.5 & 2.0 & 3.7\end{array}$ & 0.8 & 3 & 34 & 59 & & 0 & 1 & 1 & 1 \\
\hline Algiers & Algeria & $\begin{array}{lll}0.9 & 1.6 & 2.8\end{array}$ & 0.4 & 1 & 20 & 75 & 0 & 0 & 2 & 1 & 0 \\
\hline Amsterdam & Netherlands & $\begin{array}{lll}0.7 & 1.6 & 2.8\end{array}$ & 0.6 & 2 & 17 & 71 & 2 & 1 & 4 & 1 & 1 \\
\hline Andorra la Vella & Andorra & $\begin{array}{lll}0.8 & \mathbf{2 . 0} & \mathbf{3 . 8}\end{array}$ & 0.8 & 12 & 11 & 70 & 1 & 1 & 2 & 1 & 1 \\
\hline Ankara & Turkey & $\begin{array}{lll}1.5 & 2.2 & 3.9\end{array}$ & 1.0 & 22 & 20 & 51 & 2 & 2 & 1 & 1 & 1 \\
\hline Athens & Greece & $\begin{array}{lll}1.2 & 1.8 & 3.2\end{array}$ & 0.7 & 2 & 39 & 56 & 0 & 0 & 2 & 1 & 0 \\
\hline Bayrut & Lebanon & $\begin{array}{lll}1.3 & 1.8 & 3.2\end{array}$ & 0.6 & 1 & 31 & 65 & 0 & 0 & 1 & 1 & 0 \\
\hline Belgrade & Serbia and $\mathrm{M}$. & $\begin{array}{lll}1.4 & 2.2 & 3.7\end{array}$ & 0.8 & 8 & 26 & 56 & 2 & 1 & 3 & 2 & 2 \\
\hline Berlin & Germany & $\begin{array}{lll}0.8 & 1.9 & 3.4\end{array}$ & 0.8 & 4 & 22 & 65 & 2 & 1 & 4 & 1 & 2 \\
\hline Bern & Switzerland & $\begin{array}{lll}1.0 & 2.0 & 3.4\end{array}$ & 0.7 & 5 & 19 & 66 & 2 & 2 & 4 & 1 & 1 \\
\hline Bratislava & Slovakia & $\begin{array}{lll}1.3 & 2.3 & 3.8\end{array}$ & 0.9 & 9 & 22 & 60 & 2 & 1 & 3 & 2 & 2 \\
\hline Brussels & Belgium & $\begin{array}{lll}0.7 & 1.6 & 2.8\end{array}$ & 0.6 & 4 & 18 & 68 & 2 & 1 & 5 & 1 & 2 \\
\hline Bucharest & Romania & $\begin{array}{llll}1.5 & 2.4 & 4.2\end{array}$ & 0.9 & 12 & 19 & 58 & 2 & 2 & 4 & 2 & 2 \\
\hline Budapest & Hungary & $\begin{array}{lll}1.4 & 2.2 & 3.8\end{array}$ & 0.9 & 11 & 21 & 59 & 2 & 1 & 3 & 2 & 2 \\
\hline Cairo & Egypt & $\begin{array}{lll}1.2 & 1.8 & 3.4\end{array}$ & 0.7 & 6 & 27 & 64 & 1 & 1 & 1 & 1 & 1 \\
\hline Chisinau & Moldova & $\begin{array}{lll}1.5 & 2.3 & 4.2\end{array}$ & 1.0 & 12 & 19 & 58 & 2 & 1 & 4 & 2 & 2 \\
\hline Copenhagen & Denmark & $\begin{array}{lll}0.9 & 1.9 & 3.2\end{array}$ & 0.7 & 3 & 26 & 64 & 2 & 0 & 3 & 1 & 1 \\
\hline Damascus & Syria & $\begin{array}{lll}1.4 & 2.1 & 3.8\end{array}$ & 0.7 & 3 & 29 & 64 & 0 & 0 & 1 & 1 & 1 \\
\hline Douglas & Isle of Man & $\begin{array}{lll}0.4 & 1.2 & 2.3\end{array}$ & 0.5 & 3 & 16 & 73 & 2 & 0 & 4 & 1 & 1 \\
\hline Dublin & Ireland & $\begin{array}{lll}0.4 & 1.2 & 2.2\end{array}$ & 0.5 & 3 & 16 & 71 & 2 & 1 & 5 & 1 & 1 \\
\hline Gibraltar & Gibraltar & $\begin{array}{lll}0.7 & 1.4 & 2.5\end{array}$ & 0.4 & 2 & 17 & 76 & 1 & 0 & 2 & 1 & 1 \\
\hline Helsinki & Finland & $\begin{array}{lll}1.4 & 2.9 & 4.7\end{array}$ & 1.3 & 7 & 33 & 50 & 2 & 1 & 4 & 2 & 2 \\
\hline Jerusalem & Israel & $\begin{array}{lll}1.4 & 2.0 & 3.6\end{array}$ & 0.7 & 2 & 32 & 63 & 0 & 0 & 1 & 1 & 1 \\
\hline Kiev & Ukraine & $\begin{array}{lll}1.4 & 2.4 & 4.5\end{array}$ & 1.0 & 9 & 19 & 60 & 2 & 1 & 5 & 2 & 2 \\
\hline Lisbon & Portugal & $\begin{array}{llll}0.8 & 1.4 & 2.5\end{array}$ & 0.4 & 2 & 17 & 76 & 1 & 0 & 2 & 1 & 1 \\
\hline Ljubljana & Slovenia & $\begin{array}{lll}1.2 & 2.2 & 3.7\end{array}$ & 0.7 & 4 & 20 & 66 & 2 & 1 & 3 & 1 & 2 \\
\hline London & UK & $\begin{array}{lll}0.6 & 1.4 & 2.5\end{array}$ & 0.5 & 4 & 16 & 70 & 2 & 1 & 5 & 1 & 2 \\
\hline Luxemburg & Luxembourg & $\begin{array}{lll}0.8 & 1.7 & 3.0\end{array}$ & 0.6 & 4 & 20 & 67 & 2 & 1 & 4 & 1 & 1 \\
\hline Madrid & Spain & $\begin{array}{llll}0.8 & 1.6 & 2.9\end{array}$ & 0.5 & 5 & 14 & 76 & 1 & 1 & 2 & 1 & 1 \\
\hline Minsk & Belarus & $\begin{array}{lll}1.2 & 2.5 & 4.6\end{array}$ & 1.2 & 6 & 25 & 58 & 2 & 1 & 4 & 2 & 2 \\
\hline Monaco-Ville & Monaco & $\begin{array}{lll}1.0 & 1.8 & 3.1\end{array}$ & 0.5 & 3 & 21 & 71 & 1 & 1 & 2 & 1 & 1 \\
\hline Nicosia & Cyprus & $\begin{array}{lll}1.3 & 1.9 & 3.4\end{array}$ & 0.6 & 1 & 27 & 69 & 0 & 0 & 1 & 1 & 0 \\
\hline Oslo & Norway & $0.9 \quad \mathbf{2 . 3} \mathbf{4 . 0}$ & 1.1 & 7 & 31 & 54 & 2 & 0 & 3 & 1 & 1 \\
\hline Paris & France & $\begin{array}{lll}0.7 & 1.6 & 2.8\end{array}$ & 0.5 & 3 & 15 & 71 & 2 & 1 & 5 & 1 & 1 \\
\hline Prague & Czech Republic & $\begin{array}{lll}1.0 & 2.0 & 3.6\end{array}$ & 0.8 & 8 & 21 & 62 & 2 & 1 & 3 & 2 & 2 \\
\hline Rabat & Morocco & $\begin{array}{lll}0.8 & 1.4 & 2.7\end{array}$ & 0.4 & 3 & 11 & 80 & 1 & 1 & 2 & 1 & 1 \\
\hline Riga & Latvia & $\begin{array}{lll}1.0 & 2.5 & 4.4\end{array}$ & 1.3 & 6 & 28 & 55 & 2 & 1 & 5 & 2 & 2 \\
\hline Rome & Italy & $\begin{array}{lll}1.2 & 1.8 & 3.2\end{array}$ & 0.6 & 3 & 31 & 61 & 1 & 1 & 1 & 1 & 1 \\
\hline Saint Helier & Jersey & $\begin{array}{lll}0.6 & 1.2 & 2.3\end{array}$ & 0.5 & 1 & 23 & 68 & 1 & 1 & 4 & 1 & 1 \\
\hline Saint Peter Port & Guernsey and A. & $\begin{array}{lll}0.6 & 1.2 & 2.3\end{array}$ & 0.5 & 1 & 22 & 69 & 1 & 1 & 4 & 1 & 1 \\
\hline San Marino & San Marino & $\begin{array}{lll}1.2 & 2.0 & 3.4\end{array}$ & 0.7 & 5 & 27 & 61 & 1 & 1 & 2 & 1 & 1 \\
\hline Sarajevo & Bosnia and $\mathrm{H}$. & $\begin{array}{lll}1.5 & 2.3 & 3.9\end{array}$ & 0.9 & 10 & 29 & 53 & 2 & 1 & 2 & 2 & 2 \\
\hline Skopje & Macedonia & $\begin{array}{lll}1.3 & 2.1 & 3.7\end{array}$ & 0.8 & 8 & 24 & 60 & 2 & 1 & 2 & 1 & 1 \\
\hline Sofia & Bulgaria & $\begin{array}{lll}1.5 & 2.2 & 3.9\end{array}$ & 0.9 & 8 & 27 & 56 & 1 & 1 & 3 & 2 & 1 \\
\hline Stockholm & Sweden & $\begin{array}{lll}1.1 & 2.3 & 3.9\end{array}$ & 1.1 & 6 & 35 & 51 & 2 & 0 & 3 & 1 & 1 \\
\hline Tallinn & Estonia & $\begin{array}{lll}1.2 & 2.7 & 4.4\end{array}$ & 1.3 & 7 & 32 & 51 & 2 & 1 & 4 & 2 & 2 \\
\hline Tirana & Albania & $\begin{array}{lll}1.3 & 1.9 & 3.2\end{array}$ & 0.7 & 5 & 34 & 56 & 1 & 1 & 1 & 1 & 1 \\
\hline Torshavn & Faroe Islands & $0.4 \quad 1.2 \quad 2.0$ & 0.9 & 2 & 59 & 34 & 1 & 0 & 2 & 1 & 1 \\
\hline Tripoli & Libya & $\begin{array}{lll}1.2 & 1.8 & 3.3\end{array}$ & 0.6 & 3 & 26 & 67 & 1 & 0 & 2 & 1 & 1 \\
\hline Tunis & Tunisia & $\begin{array}{lll}1.1 & 1.8 & 3.1\end{array}$ & 0.5 & 2 & 24 & 70 & 0 & 0 & 2 & 1 & 1 \\
\hline Vaduz & Liechtenstein & $\begin{array}{lll}1.0 & 2.0 & 3.5\end{array}$ & 0.8 & 8 & 20 & 62 & 3 & 1 & 3 & 1 & 2 \\
\hline Valletta & Malta & $\begin{array}{lll}1.1 & 1.7 & 3.0\end{array}$ & 0.6 & 1 & 32 & 64 & 0 & 0 & 1 & 1 & 0 \\
\hline Vatican City & Vatican City & $\begin{array}{lll}1.2 & 1.8 & 3.2\end{array}$ & 0.6 & 3 & 31 & 61 & 1 & 1 & 1 & 1 & 1 \\
\hline Vienna & Austria & $\begin{array}{lll}1.2 & 2.2 & 3.7\end{array}$ & 0.8 & 7 & 21 & 62 & 2 & 1 & 3 & 2 & 2 \\
\hline Vilnius & Lithuania & $\begin{array}{lll}1.1 & 2.4 & 4.4\end{array}$ & 1.2 & 7 & 27 & 55 & 2 & 1 & 5 & 2 & 2 \\
\hline Warsaw & Poland & $\begin{array}{lll}1.0 & 2.2 & 4.0\end{array}$ & 1.1 & 7 & 29 & 53 & 3 & 1 & 4 & 1 & 2 \\
\hline Zagreb & Croatia & $\begin{array}{lll}1.2 & 2.2 & 3.8\end{array}$ & 0.9 & 8 & 23 & 60 & 2 & 2 & 3 & 2 & 2 \\
\hline
\end{tabular}


Table S12. Relative changes of total precipitation in winter (DJF) in the near future (2021-2050) compared to the period 1981-2010 (see legend of Table S10 for further details).

\begin{tabular}{|c|c|c|c|c|c|c|c|c|c|c|c|c|c|}
\hline \multirow{3}{*}{ Capital city } & \multirow{3}{*}{ Country } & \multicolumn{4}{|c|}{$B M_{k} \& B U(\%)$} & \multicolumn{8}{|c|}{$\%$ Total variance } \\
\hline & & \multicolumn{3}{|c|}{ RCP } & \multirow[b]{2}{*}{$B U$} & \multicolumn{3}{|c|}{ Main effects } & \multicolumn{3}{|c|}{ Inter. } & \multirow[b]{2}{*}{ IV } & \multirow[b]{2}{*}{ RV } \\
\hline & & 2.6 & 4.5 & 8.5 & & $\mathrm{RCM}$ & GCM & $\mathrm{RCP}$ & & I2 & I3 & & \\
\hline Amman & Jordan & -8 & -9 & -11 & 9 & 10 & 24 & 3 & 7 & 1 & 10 & 40 & 5 \\
\hline Algiers & Algeria & -2 & -6 & -8 & 6 & 11 & 19 & 9 & 4 & 2 & 8 & 41 & 6 \\
\hline Amsterdam & Netherlands & 3 & 4 & 8 & 5 & 26 & 19 & 13 & 3 & 1 & 4 & 30 & 3 \\
\hline Andorra la Vella & Andorra & 3 & 1 & -2 & 8 & 20 & 19 & 8 & 8 & 2 & 6 & 31 & 5 \\
\hline Ankara & Turkey & 2 & 0 & 1 & 7 & 15 & 40 & 1 & 4 & 1 & 4 & 33 & 2 \\
\hline Athens & Greece & 0 & -3 & -2 & 9 & 6 & 56 & 3 & 2 & 1 & 2 & 27 & 3 \\
\hline Bayrut & Lebanon & -2 & -3 & -4 & 8 & 15 & 39 & 1 & 6 & 0 & 8 & 29 & 3 \\
\hline Belgrade & Serbia and $\mathrm{M}$. & 5 & 6 & 7 & 7 & 17 & 34 & 2 & 11 & 1 & 6 & 24 & 6 \\
\hline Berlin & Germany & 1 & 4 & 8 & 6 & 23 & 28 & 17 & 2 & 1 & 4 & 22 & 3 \\
\hline Bern & Switzerland & 4 & 6 & 8 & 6 & 3 & 39 & 7 & 6 & 1 & 6 & 34 & 5 \\
\hline Bratislava & Slovakia & 6 & 6 & 10 & 8 & 17 & 32 & 6 & 9 & 1 & 3 & 26 & 5 \\
\hline Brussels & Belgium & 3 & 4 & 7 & 5 & 24 & 17 & 12 & 3 & 1 & 5 & 36 & 2 \\
\hline Bucharest & Romania & 5 & 5 & 7 & 9 & 25 & 18 & 1 & 9 & 3 & 8 & 30 & 5 \\
\hline Budapest & Hungary & 5 & 6 & 8 & 7 & 14 & 28 & 2 & 10 & 1 & 7 & 34 & 4 \\
\hline Cairo & Egypt & -10 & -3 & -10 & 14 & 19 & 20 & 5 & 9 & 2 & 8 & 31 & 6 \\
\hline Chisinau & Moldova & 4 & 3 & 7 & 8 & 17 & 27 & 4 & 4 & 3 & 6 & 33 & 7 \\
\hline Copenhagen & Denmark & 2 & 4 & 7 & 5 & 23 & 10 & 12 & 11 & 2 & 3 & 31 & 9 \\
\hline Damascus & Syria & -4 & -5 & -7 & 9 & 19 & 27 & 3 & 4 & 2 & 7 & 33 & 5 \\
\hline Douglas & Isle of Man & 3 & 4 & 7 & 4 & 22 & 8 & 14 & 11 & 4 & 9 & 27 & 5 \\
\hline Dublin & Ireland & 4 & 4 & 6 & 6 & 43 & 11 & 3 & 12 & 3 & 4 & 20 & 5 \\
\hline Gibraltar & Gibraltar & 0 & -6 & -13 & 9 & 20 & 9 & 25 & 7 & 0 & 3 & 33 & 3 \\
\hline Helsinki & Finland & 1 & 3 & 6 & 5 & 20 & 26 & 11 & 3 & 2 & 4 & 31 & 4 \\
\hline Jerusalem & Israel & -8 & -10 & -11 & 8 & 23 & 14 & 2 & 6 & 2 & 9 & 39 & 4 \\
\hline Kiev & Ukraine & 3 & 4 & 7 & 6 & 12 & 39 & 6 & 6 & 0 & 4 & 28 & 4 \\
\hline Lisbon & Portugal & 1 & -2 & -7 & 8 & 7 & 28 & 15 & 2 & 1 & 7 & 37 & 2 \\
\hline Ljubljana & Slovenia & 7 & 5 & 8 & 7 & 11 & 16 & 4 & 7 & 1 & 11 & 44 & 5 \\
\hline London & UK & 3 & 4 & 7 & 5 & 24 & 15 & 13 & 5 & 1 & 5 & 34 & 3 \\
\hline Luxemburg & Luxembourg & 4 & 5 & 7 & 5 & 32 & 16 & 7 & 2 & 1 & 4 & 35 & 3 \\
\hline Madrid & Spain & 3 & 1 & -4 & 8 & 8 & 25 & 12 & 3 & 1 & 9 & 40 & 2 \\
\hline Minsk & Belarus & 3 & 5 & 8 & 5 & 25 & 21 & 15 & 4 & 1 & 2 & 28 & 4 \\
\hline Monaco-Ville & Monaco & 9 & 5 & 1 & 10 & 9 & 19 & 10 & 5 & 1 & 14 & 36 & 5 \\
\hline Nicosia & Cypr & -3 & -5 & -9 & 7 & 9 & 18 & 10 & 8 & 2 & 8 & 40 & 5 \\
\hline Oslo & Norway & 2 & 5 & 9 & 5 & 13 & 20 & 18 & 5 & 2 & 5 & 34 & 3 \\
\hline Paris & France & 4 & 5 & 8 & 5 & 30 & 17 & 10 & 2 & 1 & 2 & 34 & 3 \\
\hline Prague & Czech Republic & 4 & 7 & 11 & 7 & 26 & 33 & 13 & 2 & 1 & 5 & 18 & 3 \\
\hline Rabat & Morocco & 0 & -10 & -16 & 9 & 12 & 12 & 31 & 4 & 1 & 6 & 32 & 3 \\
\hline Riga & Latvia & 1 & 3 & 6 & 5 & 26 & 19 & 13 & 5 & 2 & 2 & 28 & 5 \\
\hline Rome & Italy & 6 & 0 & 1 & 7 & 10 & 25 & 11 & 5 & 1 & 6 & 38 & 4 \\
\hline Saint Helier & Jersey & 1 & 2 & 5 & 5 & 12 & 23 & 10 & 5 & 1 & 2 & 42 & 5 \\
\hline Saint Peter Port & Guernsey and A. & 2 & 3 & 6 & 4 & 11 & 23 & 10 & 5 & 1 & 3 & 43 & 3 \\
\hline San Marino & San Marino & 2 & 0 & 2 & 9 & 17 & 17 & 2 & 13 & 1 & 7 & 34 & 10 \\
\hline Sarajevo & Bosnia and $\mathrm{H}$. & 3 & 4 & 5 & 8 & 42 & 23 & 2 & 4 & 3 & 4 & 19 & 2 \\
\hline Skopje & Macedonia & 4 & 2 & 5 & 9 & 27 & 26 & 2 & 10 & 1 & 2 & 27 & 5 \\
\hline Sofia & Bulgaria & 2 & 2 & 5 & 7 & 25 & 15 & 5 & 11 & 1 & 5 & 33 & 5 \\
\hline Stockholm & Sweden & 2 & 4 & 6 & 5 & 29 & 25 & 10 & 3 & 2 & 2 & 27 & 3 \\
\hline Tallinn & Estonia & 2 & 3 & 6 & 6 & 37 & 24 & 8 & 5 & 1 & 2 & 20 & 3 \\
\hline Tirana & Albania & 4 & 1 & 1 & 6 & 10 & 25 & 4 & 7 & 2 & 8 & 40 & 4 \\
\hline Torshavn & Faroe Islands & -2 & 0 & -1 & 6 & 29 & 38 & 1 & 7 & 2 & 3 & 16 & 3 \\
\hline Tripoli & Libya & -6 & -8 & -8 & 13 & 14 & 56 & 1 & 3 & 1 & 2 & 19 & 3 \\
\hline Tunis & Tunisia & -3 & -6 & -7 & 9 & 12 & 37 & 3 & 7 & 1 & 3 & 32 & 6 \\
\hline Vaduz & Liechtenstein & 2 & 4 & 6 & 7 & 9 & 37 & 4 & 8 & 1 & 6 & 30 & 5 \\
\hline Valletta & Malta & -3 & -7 & -7 & 9 & 28 & 30 & 5 & 4 & 1 & 2 & 26 & 4 \\
\hline Vatican City & Vatican City & 6 & 0 & 1 & 7 & 10 & 25 & 11 & 5 & 1 & 6 & 38 & 4 \\
\hline Vienna & Austria & 4 & 4 & 9 & 8 & 21 & 31 & 7 & 7 & 1 & 2 & 24 & 6 \\
\hline Vilnius & Lithuania & 2 & 3 & 7 & 4 & 25 & 16 & 21 & 3 & 1 & 2 & 29 & 3 \\
\hline Warsaw & Poland & 2 & 4 & 8 & 5 & 22 & 29 & 17 & 3 & 1 & 2 & 23 & 4 \\
\hline Zagreb & Croatia & 8 & 7 & 10 & 7 & 11 & 27 & 4 & 6 & 1 & 9 & 36 & 6 \\
\hline
\end{tabular}


Table S13. Relative changes of total precipitation in winter (DJF) at the end of the century (2071-2099) compared to the period 1981-2010 (see legend of Table S10 for further details).

\begin{tabular}{|c|c|c|c|c|c|c|c|c|c|c|c|c|c|}
\hline \multirow{3}{*}{ Capital city } & \multirow{3}{*}{ Country } & \multicolumn{4}{|c|}{$B M_{k} \& B U(\%)$} & \multicolumn{8}{|c|}{$\%$ Total variance } \\
\hline & & \multicolumn{3}{|c|}{ RCP } & \multirow{2}{*}{$B U$} & \multicolumn{3}{|c|}{ Main effects } & \multicolumn{3}{|c|}{ Inter. } & \multirow[b]{2}{*}{ IV } & \multirow[b]{2}{*}{ RV } \\
\hline & & 2.6 & 4.5 & 8.5 & & $\mathrm{RCM}$ & GCM & $\mathrm{RCP}$ & & I2 & I3 & & \\
\hline Amman & Jordan & -19 & -18 & -29 & 15 & 13 & 40 & 10 & 8 & 2 & 9 & 12 & 6 \\
\hline Algiers & Algeria & -1 & -12 & -17 & 14 & 11 & 31 & 19 & 5 & 3 & 17 & 9 & 6 \\
\hline Amsterdam & Netherlands & 5 & 9 & 20 & 10 & 28 & 22 & 28 & 3 & 2 & 7 & 7 & 4 \\
\hline Andorra la Vella & Andorra & 9 & 2 & -8 & 14 & 24 & 17 & 21 & 9 & 4 & 11 & 8 & 7 \\
\hline Ankara & Turkey & 3 & 0 & 0 & 12 & 19 & 53 & 2 & 6 & 1 & 3 & 10 & 6 \\
\hline Athens & Greece & 1 & -8 & -8 & 16 & 4 & 62 & 7 & 3 & 4 & 4 & 9 & 6 \\
\hline Bayrut & Lebanon & -7 & -6 & -13 & 15 & 19 & 56 & 4 & 5 & 1 & 5 & 7 & 3 \\
\hline Belgrade & Serbia and $\mathrm{M}$. & 7 & 11 & 15 & 14 & 21 & 36 & 5 & 11 & 1 & 9 & 7 & 9 \\
\hline Berlin & Germany & 1 & 9 & 19 & 10 & 23 & 21 & 34 & 3 & 1 & 7 & 6 & 5 \\
\hline Bern & Switzerland & 7 & 13 & 18 & 10 & 4 & 44 & 18 & 5 & 2 & 7 & 11 & 9 \\
\hline Bratislava & Slovakia & 11 & 9 & 22 & 14 & 13 & 42 & 13 & 10 & 1 & 6 & 7 & 7 \\
\hline Brussels & Belgium & 5 & 9 & 19 & 9 & 26 & 13 & 34 & 4 & 2 & 10 & 10 & 2 \\
\hline Bucharest & Romania & 10 & 8 & 16 & 18 & 28 & 20 & 3 & 13 & 5 & 15 & 8 & 8 \\
\hline Budapest & Hungary & 12 & 9 & 19 & 14 & 14 & 34 & 8 & 12 & 3 & 13 & 8 & 8 \\
\hline Cairo & Egypt & -23 & -9 & -26 & 24 & 17 & 26 & 9 & 13 & 4 & 12 & 11 & 9 \\
\hline Chisinau & Moldova & 7 & 3 & 14 & 14 & 15 & 22 & 11 & 8 & 5 & 17 & 11 & 11 \\
\hline Copenhagen & Denmark & 4 & 9 & 18 & 11 & 26 & 11 & 23 & 13 & 3 & 6 & 6 & 12 \\
\hline Damascus & Syria & -11 & -14 & -21 & 16 & 22 & 40 & 7 & 3 & 5 & 6 & 9 & 9 \\
\hline Douglas & Isle of Man & 6 & 10 & 17 & 9 & 21 & 17 & 22 & 11 & 5 & 11 & 6 & 6 \\
\hline Dublin & Ireland & 8 & 9 & 14 & 13 & 51 & 12 & 5 & 14 & 3 & 5 & 5 & 6 \\
\hline Gibraltar & Gibraltar & 4 & -15 & -32 & 13 & 15 & 7 & 55 & 5 & 1 & 6 & 9 & 3 \\
\hline Helsinki & Finland & 2 & 7 & 16 & 9 & 21 & 24 & 28 & 2 & 2 & 9 & 7 & 6 \\
\hline Jerusalem & Israel & -20 & -20 & -30 & 14 & 20 & 36 & 9 & 8 & 3 & 6 & 12 & 6 \\
\hline Kiev & Ukraine & 6 & 6 & 15 & 11 & 14 & 38 & 14 & 8 & 1 & 8 & 9 & 8 \\
\hline Lisbon & Portugal & 6 & -4 & -19 & 15 & 6 & 33 & 31 & 3 & 2 & 14 & 8 & 4 \\
\hline Ljubljana & Slovenia & 18 & 9 & 20 & 13 & 12 & 24 & 13 & 11 & 1 & 19 & 12 & 8 \\
\hline London & UK & 5 & 7 & 17 & 9 & 31 & 17 & 23 & 5 & 2 & 9 & 9 & 3 \\
\hline Luxemburg & Luxembourg & 6 & 10 & 18 & 10 & 38 & 18 & 19 & 3 & 1 & 8 & 9 & 4 \\
\hline Madrid & Spain & 11 & 0 & -12 & 15 & 13 & 34 & 27 & 3 & 2 & 8 & 10 & 3 \\
\hline Minsk & Belarus & 5 & 9 & 20 & 10 & 31 & 15 & 31 & 3 & 2 & 6 & 6 & 5 \\
\hline Monaco-Ville & Monaco & 23 & 8 & -1 & 20 & 11 & 23 & 19 & 7 & 2 & 22 & 9 & 8 \\
\hline Nicosia & Cypr & -9 & -15 & -24 & 14 & 15 & 35 & 16 & 7 & 3 & 7 & 10 & 7 \\
\hline Oslo & Norway & 5 & 10 & 21 & 9 & 18 & 19 & 34 & 5 & 3 & 7 & 10 & 4 \\
\hline Paris & France & 7 & 11 & 19 & 9 & 32 & 20 & 22 & 3 & 2 & 7 & 9 & 5 \\
\hline Prague & Czech Republic & 5 & 12 & 24 & 13 & 26 & 31 & 26 & 3 & 1 & 4 & 5 & 5 \\
\hline Rabat & Morocco & 6 & -23 & -38 & 15 & 11 & 7 & 59 & 4 & 1 & 9 & 7 & 3 \\
\hline Riga & Latvia & 2 & 7 & 17 & 10 & 38 & 13 & 28 & 3 & 2 & 5 & 6 & 5 \\
\hline Rome & Italy & 12 & -4 & 0 & 13 & 16 & 22 & 21 & 8 & 2 & 15 & 10 & 7 \\
\hline Saint Helier & Jersey & 4 & 4 & 13 & 8 & 17 & 26 & 20 & 6 & 2 & 9 & 12 & 8 \\
\hline Saint Peter Port & Guernsey and A. & 5 & 5 & 13 & 8 & 13 & 33 & 16 & 6 & 2 & 13 & 12 & 6 \\
\hline San Marino & San Marino & 7 & 0 & 4 & 16 & 21 & 23 & 4 & 14 & 1 & 14 & 11 & 13 \\
\hline Sarajevo & Bosnia and $\mathrm{H}$. & 3 & 6 & 12 & 15 & 46 & 21 & 7 & 5 & 6 & 7 & 5 & 4 \\
\hline Skopje & Macedonia & 9 & 3 & 12 & 17 & 37 & 26 & 5 & 12 & 1 & 3 & 7 & 9 \\
\hline Sofia & Bulgaria & 4 & 3 & 12 & 13 & 29 & 27 & 8 & 14 & 1 & 6 & 8 & 7 \\
\hline Stockholm & Sweden & 3 & 7 & 16 & 10 & 32 & 26 & 23 & 4 & 2 & 4 & 6 & 3 \\
\hline Tallinn & Estonia & 1 & 7 & 18 & 13 & 40 & 19 & 24 & 3 & 2 & 5 & 4 & 2 \\
\hline Tirana & Albania & 7 & 2 & 4 & 11 & 20 & 25 & 3 & 12 & 2 & 16 & 12 & 8 \\
\hline Torshavn & Faroe Islands & -3 & -1 & -1 & 11 & 23 & 52 & 1 & 10 & 3 & 4 & 4 & 4 \\
\hline Tripoli & Libya & -14 & -15 & -20 & 26 & 16 & 67 & 1 & 3 & 2 & 4 & 5 & 3 \\
\hline Tunis & Tunisia & -8 & -12 & -16 & 16 & 13 & 51 & 4 & 7 & 3 & 7 & 9 & 6 \\
\hline Vaduz & Liechtenstein & 0 & 8 & 13 & 13 & 10 & 41 & 15 & 12 & 1 & 6 & 9 & 7 \\
\hline Valletta & Malta & -6 & -13 & -19 & 17 & 31 & 36 & 9 & 5 & 2 & 6 & 7 & 5 \\
\hline Vatican City & Vatican City & 12 & -4 & 0 & 13 & 16 & 22 & 21 & 8 & 2 & 15 & 10 & 7 \\
\hline Vienna & Austria & 7 & 5 & 20 & 15 & 18 & 40 & 16 & 7 & 1 & 5 & 6 & 9 \\
\hline Vilnius & Lithuania & 2 & 7 & 18 & 8 & 31 & 13 & 37 & 3 & 1 & 4 & 6 & 5 \\
\hline Warsaw & Poland & 3 & 10 & 18 & 10 & 27 & 27 & 29 & 2 & 1 & 3 & 6 & 5 \\
\hline Zagreb & Croatia & 17 & 12 & 24 & 13 & 15 & 24 & 13 & 8 & 1 & 19 & 10 & 10 \\
\hline
\end{tabular}


Table S14. Absolute changes of mean temperature in summer (JJA) in the near future (2021-2050) compared to the period 1981-2010 (see legend of Table S10 for further details).

\begin{tabular}{|c|c|c|c|c|c|c|c|c|c|c|}
\hline \multirow{3}{*}{ Capital city } & \multirow[b]{3}{*}{ Country } & \multicolumn{2}{|c|}{$B M_{k} \& B U\left({ }^{\circ} \mathrm{C}\right)$} & \multicolumn{7}{|c|}{ \% Total variance } \\
\hline & & RCP & \multirow{2}{*}{$B U$} & \multicolumn{3}{|c|}{ Main effects } & \multicolumn{2}{|c|}{ Inter. } & \multirow[b]{2}{*}{ IV } & \\
\hline & & $\begin{array}{lll}2.6 & 4.5 & 8.5\end{array}$ & & RCM & GCM & $\mathrm{RCP}$ & I1 I & I2 I3 & & RV \\
\hline Amman & Jordan & $\begin{array}{lll}1.2 & 1.5 & 1.9\end{array}$ & 0.5 & 6 & 60 & 28 & 1 & \begin{tabular}{ll|}
0 & 1
\end{tabular} & 3 & $\overline{0}$ \\
\hline Algiers & Algeria & $\begin{array}{lll}0.9 & 1.1 & 1.3\end{array}$ & 0.3 & 3 & 66 & 23 & 1 & 0 & 4 & 1 \\
\hline Amsterdam & Netherlands & $\begin{array}{lll}0.8 & 0.9 & 1.1\end{array}$ & 0.4 & 12 & 62 & 11 & 4 & 1 & 6 & 1 \\
\hline Andorra la Vella & Andorra & $\begin{array}{lll}1.1 & 1.5 & 1.8\end{array}$ & 0.5 & 18 & 44 & 24 & 5 & 1 & 4 & 2 \\
\hline Ankara & Turkey & 1.31 .62 .1 & 0.5 & 14 & 52 & 27 & 2 & 1 & 3 & 1 \\
\hline Athens & Greece & $\begin{array}{lll}1.2 & 1.5 & 1.9\end{array}$ & 0.5 & 18 & 49 & 25 & 2 & 0 & 3 & 1 \\
\hline Bayrut & Lebanon & $\begin{array}{lll}1.0 & 1.1 & 1.5\end{array}$ & 0.3 & 3 & 63 & 28 & 1 & 0 & 3 & 0 \\
\hline Belgrade & Serbia and $\mathrm{M}$. & $\begin{array}{lll}1.2 & 1.5 & 1.9\end{array}$ & 0.7 & 29 & 46 & 14 & 4 & 0 & 4 & 1 \\
\hline Berlin & Germany & $\begin{array}{lll}0.9 & 1.1 & 1.3\end{array}$ & 0.5 & 30 & 47 & 8 & 4 & 1 & 6 & 3 \\
\hline Bern & Switzerland & $\begin{array}{lll}1.0 & 1.3 & 1.5\end{array}$ & 0.5 & 29 & 44 & 12 & 6 & 1 & 5 & 2 \\
\hline Bratislava & Slovakia & $\begin{array}{lll}1.1 & 1.3 & 1.5\end{array}$ & 0.6 & 35 & 43 & 10 & 3 & 1 & 5 & 2 \\
\hline Brussels & Belgium & $\begin{array}{lll}0.8 & 1.0 & 1.2\end{array}$ & 0.5 & 26 & 47 & 10 & 5 & 1 & 7 & 2 \\
\hline Bucharest & Romania & $\begin{array}{lll}1.2 & 1.5 & 1.9\end{array}$ & 0.7 & 27 & 49 & 16 & 3 & 0 & 3 & 1 \\
\hline Budapest & Hungary & $\begin{array}{lll}1.1 & 1.4 & 1.6\end{array}$ & 0.7 & 38 & 42 & 9 & 3 & 1 & 5 & 2 \\
\hline Cairo & Egypt & $\begin{array}{lll}1.2 & 1.4 & 1.8\end{array}$ & 0.4 & 4 & 61 & 30 & 1 & 0 & 2 & 0 \\
\hline Chisinau & Moldova & $\begin{array}{lll}1.2 & 1.4 & 1.8\end{array}$ & 0.7 & 30 & 47 & 13 & 3 & 0 & 4 & 2 \\
\hline Copenhagen & Denmark & $\begin{array}{lll}0.9 & 1.0 & 1.3\end{array}$ & 0.4 & 17 & 59 & 13 & 2 & 1 & 6 & 1 \\
\hline Damascus & Syria & $\begin{array}{lll}1.3 & 1.6 & 2.0\end{array}$ & 0.4 & 5 & 56 & 33 & 1 & 0 & 3 & 0 \\
\hline Douglas & Isle of Man & $\begin{array}{lll}0.6 & 0.7 & 0.9\end{array}$ & 0.4 & 12 & 66 & 10 & 2 & 0 & 5 & 1 \\
\hline Dublin & Ireland & $\begin{array}{lll}0.6 & 0.7 & 0.9\end{array}$ & 0.4 & 16 & 57 & 14 & 2 & 1 & 6 & 2 \\
\hline Gibraltar & Gibraltar & $\begin{array}{lll}0.7 & 0.9 & 1.1\end{array}$ & 0.3 & 18 & 38 & 28 & 3 & 1 & 5 & 2 \\
\hline Helsinki & Finland & $\begin{array}{lll}1.1 & 1.2 & 1.6\end{array}$ & 0.4 & 15 & 55 & 18 & 2 & 1 & 6 & 1 \\
\hline Jerusalem & Israel & $\begin{array}{lll}1.2 & 1.4 & 1.8\end{array}$ & 0.4 & 8 & 60 & 27 & 1 & 0 & 3 & 1 \\
\hline Kiev & Ukraine & $\begin{array}{lll}1.1 & 1.2 & 1.7\end{array}$ & 0.7 & 34 & 45 & 10 & 4 & 0 & 4 & 2 \\
\hline Lisbon & Portugal & $\begin{array}{lll}0.8 & 1.0 & 1.2\end{array}$ & 0.3 & 10 & 48 & 26 & 2 & 1 & 8 & 2 \\
\hline Ljubljana & Slovenia & $\begin{array}{lll}1.1 & 1.4 & 1.7\end{array}$ & 0.6 & 31 & 45 & 12 & 4 & 1 & 4 & 2 \\
\hline London & UK & $\begin{array}{llll}0.8 & 0.9 & 1.1\end{array}$ & 0.4 & 18 & 52 & 14 & 4 & 1 & 6 & 2 \\
\hline Luxemburg & Luxembourg & $\begin{array}{lll}0.9 & 1.1 & 1.3\end{array}$ & 0.5 & 33 & 42 & 10 & 6 & 1 & 6 & 2 \\
\hline Madrid & Spain & $\begin{array}{lll}1.1 & 1.6 & 1.9\end{array}$ & 0.5 & 13 & 46 & 29 & 2 & 1 & 4 & 2 \\
\hline Minsk & Belarus & $\begin{array}{lll}1.1 & 1.2 & 1.5\end{array}$ & 0.6 & 37 & 43 & 8 & 4 & 0 & 4 & 2 \\
\hline Monaco-Ville & Monaco & $\begin{array}{lll}1.1 & 1.4 & 1.6\end{array}$ & 0.5 & 12 & 54 & 20 & 5 & 1 & 5 & 2 \\
\hline Nicosia & Cyp & $\begin{array}{lll}1.1 & 1.4 & 1.8\end{array}$ & 0.4 & 3 & 54 & 37 & 1 & 1 & 3 & 0 \\
\hline Oslo & Nor & $\begin{array}{llll}0.9 & 1.0 & 1.4\end{array}$ & 0.4 & 22 & 48 & 19 & 2 & 1 & 6 & 1 \\
\hline Paris & France & $\begin{array}{lll}0.9 & 1.1 & 1.3\end{array}$ & 0.5 & 29 & 40 & 11 & 6 & 1 & 7 & 3 \\
\hline Prague & Czech Republic & $\begin{array}{lll}1.0 & 1.2 & 1.4\end{array}$ & 0.6 & 32 & 45 & 9 & 5 & 1 & 5 & 3 \\
\hline Rabat & Morocco & $\begin{array}{lll}0.7 & 0.8 & 1.1\end{array}$ & 0.3 & 12 & 44 & 21 & 6 & 1 & 8 & 3 \\
\hline Riga & Latvia & $\begin{array}{lll}1.0 & 1.1 & 1.4\end{array}$ & 0.5 & 16 & 57 & 14 & 3 & 0 & 6 & 2 \\
\hline Rome & Italy & $\begin{array}{lll}1.1 & 1.5 & 1.7\end{array}$ & 0.4 & 15 & 46 & 24 & 5 & 1 & 5 & 2 \\
\hline Saint Helier & Jersey & $\begin{array}{llll}0.7 & 0.9 & 1.1\end{array}$ & 0.4 & 10 & 66 & 14 & 1 & 1 & 5 & 1 \\
\hline Saint Peter Port & Guernsey and A. & $\begin{array}{llll}0.7 & 0.8 & 1.0\end{array}$ & 0.3 & 2 & 73 & 15 & 1 & 0 & 5 & 1 \\
\hline San Marino & San Marino & $\begin{array}{lll}1.1 & 1.5 & 1.7\end{array}$ & 0.6 & 20 & 51 & 16 & 4 & 1 & 4 & 1 \\
\hline Sarajevo & Bosnia and $\mathrm{H}$. & $\begin{array}{lll}1.2 & 1.5 & 1.8\end{array}$ & 0.6 & 25 & 51 & 16 & 3 & 1 & 3 & 1 \\
\hline Skopje & Macedonia & $\begin{array}{lll}1.3 & 1.6 & 2.0\end{array}$ & 0.6 & 19 & 55 & 19 & 2 & 0 & 3 & 1 \\
\hline Sofia & Bulgaria & $1.2 \quad 1.6 \quad 2.0$ & 0.6 & 18 & 54 & 20 & 3 & 0 & 3 & 1 \\
\hline Stockholm & Sweden & $\begin{array}{lll}1.0 & 1.2 & 1.5\end{array}$ & 0.4 & 21 & 52 & 16 & 3 & 1 & 6 & 1 \\
\hline Tallinn & Estonia & $\begin{array}{lll}1.1 & 1.3 & 1.6\end{array}$ & 0.4 & 11 & 60 & 18 & 2 & 1 & 6 & 1 \\
\hline Tirana & Albania & $\begin{array}{lll}1.2 & 1.6 & 1.9\end{array}$ & 0.5 & 18 & 47 & 22 & 4 & 1 & 4 & 1 \\
\hline Torshavn & Faroe Islands & $\begin{array}{lll}0.5 & 0.7 & 0.9\end{array}$ & 0.3 & 5 & 66 & 17 & 1 & 0 & 7 & 1 \\
\hline Tripoli & Libya & $\begin{array}{lll}1.1 & 1.2 & 1.7\end{array}$ & 0.4 & 6 & 51 & 34 & 2 & 1 & 4 & 1 \\
\hline Tunis & Tunisia & $\begin{array}{lll}1.1 & 1.3 & 1.6\end{array}$ & 0.4 & 8 & 53 & 29 & 2 & 0 & 4 & 1 \\
\hline Vaduz & Liechtenstein & $\begin{array}{lll}1.0 & 1.3 & 1.5\end{array}$ & 0.5 & 23 & 45 & 15 & 7 & 1 & 6 & 2 \\
\hline Valletta & Malta & $\begin{array}{lll}1.1 & 1.3 & 1.6\end{array}$ & 0.3 & 8 & 56 & 29 & 1 & 0 & 3 & 0 \\
\hline Vatican City & Vatican City & $\begin{array}{lll}1.1 & 1.5 & 1.7\end{array}$ & 0.4 & 15 & 46 & 24 & 5 & 1 & 5 & 2 \\
\hline Vienna & Austria & $\begin{array}{lll}1.0 & 1.3 & 1.5\end{array}$ & 0.6 & 33 & 45 & 10 & 4 & 1 & 5 & 2 \\
\hline Vilnius & Lithuania & $\begin{array}{lll}1.1 & 1.2 & 1.5\end{array}$ & 0.6 & 34 & 45 & 9 & 4 & 0 & 5 & 2 \\
\hline Warsaw & Poland & $\begin{array}{lll}1.0 & 1.2 & 1.4\end{array}$ & 0.6 & 38 & 44 & 7 & 2 & 0 & 5 & 3 \\
\hline Zagreb & Croatia & $\begin{array}{lll}1.1 & 1.5 & 1.7\end{array}$ & 0.6 & 33 & 43 & 12 & 4 & 11 & 5 & 2 \\
\hline
\end{tabular}


Table S15. Absolute changes of mean temperature in summer (JJA) at the end of the century (2071-2099) compared to the period 1981-2010 (see legend of Table S10 for further details).

\begin{tabular}{|c|c|c|c|c|c|c|c|c|c|c|}
\hline \multirow{3}{*}{ Capital city } & \multirow[b]{3}{*}{ Country } & \multicolumn{2}{|c|}{$B M_{k} \& B U\left({ }^{\circ} \mathrm{C}\right)$} & \multicolumn{7}{|c|}{ \% Total variance } \\
\hline & & RCP & \multirow{2}{*}{$B U$} & \multicolumn{3}{|c|}{ Main effects } & \multicolumn{2}{|c|}{ Inter. } & \multirow[b]{2}{*}{ IV } & \\
\hline & & $\begin{array}{lll}2.6 & 4.5 & 8.5\end{array}$ & & RCM & GCM & $\mathrm{RCP}$ & I1 I & I2 I3 & & RV \\
\hline Amman & Jordan & 1.62 .85 .0 & 0.8 & 3 & 20 & 74 & 1 & \begin{tabular}{ll|}
0 & 1
\end{tabular} & 0 & $\overline{0}$ \\
\hline Algiers & Algeria & 1.22 .13 .6 & 0.7 & 2 & 29 & 67 & 0 & 0 & 0 & 0 \\
\hline Amsterdam & Netherlands & $\begin{array}{lll}1.0 & 1.7 & 3.0\end{array}$ & 0.8 & 9 & 30 & 56 & 2 & 0 & 1 & 1 \\
\hline Andorra la Vella & Andorra & 1.42 .85 .1 & 1.1 & 13 & 17 & 64 & 2 & 1 & 0 & 1 \\
\hline Ankara & Turkey & $\begin{array}{lll}1.9 & 3.2 & 5.7\end{array}$ & 1.1 & 7 & 23 & 67 & 0 & 0 & 0 & 0 \\
\hline Athens & Greece & $\begin{array}{lll}1.6 & 2.8 & 5.0\end{array}$ & 0.9 & 10 & 19 & 68 & 1 & 0 & 0 & 1 \\
\hline Bayrut & Lebanon & $\begin{array}{lll}1.3 & 2.1 & 3.7\end{array}$ & 0.6 & 1 & 22 & 75 & 0 & 0 & 0 & 0 \\
\hline Belgrade & Serbia and $\mathrm{M}$. & $\begin{array}{lll}1.7 & 2.9 & 5.0\end{array}$ & 1.3 & 18 & 22 & 52 & 3 & 1 & 1 & 1 \\
\hline Berlin & Germany & $\begin{array}{lll}1.2 & 2.0 & 3.5\end{array}$ & 1.0 & 24 & 24 & 45 & 3 & 0 & 1 & 2 \\
\hline Bern & Switzerland & $\begin{array}{lll}1.3 & 2.4 & 4.3\end{array}$ & 1.3 & 22 & 22 & 48 & 4 & 1 & 1 & 2 \\
\hline Bratislava & Slovakia & $\begin{array}{lll}1.5 & 2.5 & 4.3\end{array}$ & 1.3 & 25 & 24 & 44 & 2 & 1 & 1 & 2 \\
\hline Brussels & Belgium & $\begin{array}{lll}1.0 & 1.9 & 3.4\end{array}$ & 0.9 & 17 & 22 & 53 & 3 & 1 & 1 & 1 \\
\hline Bucharest & Romania & $\begin{array}{lll}1.7 & 3.0 & 5.2\end{array}$ & 1.2 & 16 & 21 & 57 & 2 & 1 & 0 & 1 \\
\hline Budapest & Hungary & $1.6 \quad 2.74 .6$ & 1.4 & 27 & 22 & 44 & 2 & 1 & 1 & 2 \\
\hline Cairo & Egypt & $\begin{array}{lll}1.6 & 2.7 & 4.7\end{array}$ & 0.8 & 2 & 25 & 71 & 1 & 0 & 0 & 0 \\
\hline Chisinau & Moldova & $\begin{array}{lll}1.8 & 2.8 & 4.9\end{array}$ & 1.3 & 22 & 23 & 49 & 2 & 1 & 1 & 1 \\
\hline Copenhagen & Denmark & $\begin{array}{lll}1.2 & 2.0 & 3.3\end{array}$ & 0.8 & 13 & 30 & 52 & 1 & 0 & 1 & 1 \\
\hline Damascus & Syria & $\begin{array}{lll}1.6 & 3.0 & 5.3\end{array}$ & 0.8 & 2 & 18 & 77 & 0 & 0 & 0 & 0 \\
\hline Douglas & Isle of Man & $\begin{array}{lll}0.8 & 1.5 & 2.7\end{array}$ & 0.7 & 7 & 34 & 54 & 1 & 0 & 1 & 1 \\
\hline Dublin & Ireland & $\begin{array}{llll}0.7 & 1.5 & 2.8\end{array}$ & 0.7 & 8 & 29 & 59 & 1 & 0 & 1 & 1 \\
\hline Gibraltar & Gibraltar & $\begin{array}{lll}0.9 & 1.8 & 3.0\end{array}$ & 0.5 & 7 & 12 & 76 & 1 & 0 & 1 & 1 \\
\hline Helsinki & Finland & $\begin{array}{lll}1.6 & 2.5 & 4.0\end{array}$ & 0.9 & 15 & 26 & 52 & 1 & 1 & 1 & 2 \\
\hline Jerusalem & Israel & $\begin{array}{lll}1.5 & 2.7 & 4.8\end{array}$ & 0.8 & 4 & 22 & 71 & 1 & 0 & 0 & 0 \\
\hline Kiev & Ukraine & $\begin{array}{lll}1.7 & 2.6 & 4.5\end{array}$ & 1.3 & 29 & 20 & 43 & 2 & 1 & 1 & 2 \\
\hline Lisbon & Portugal & $\begin{array}{lll}0.9 & 1.9 & 3.3\end{array}$ & 0.4 & 4 & 9 & 83 & 1 & 0 & 1 & 1 \\
\hline Ljubljana & Slovenia & $\begin{array}{lll}1.5 & 2.7 & 4.7\end{array}$ & 1.4 & 23 & 24 & 46 & 3 & 1 & 1 & 2 \\
\hline London & UK & $\begin{array}{lll}0.9 & 1.8 & 3.3\end{array}$ & 0.8 & 13 & 22 & 59 & 2 & 0 & 1 & 1 \\
\hline Luxemburg & Luxembourg & $\begin{array}{lll}1.2 & 2.1 & 3.8\end{array}$ & 1.1 & 24 & 18 & 50 & 3 & 1 & 1 & 2 \\
\hline Madrid & Spain & 1.43 .05 .6 & 1.0 & 8 & 16 & 72 & 1 & 0 & 0 & 1 \\
\hline Minsk & Belarus & $\begin{array}{lll}1.7 & 2.5 & 4.1\end{array}$ & 1.3 & 34 & 20 & 39 & 2 & 1 & 1 & 2 \\
\hline Monaco-Ville & Monaco & $1.3 \quad 2.64 .6$ & 0.9 & 7 & 20 & 67 & 2 & 1 & 0 & 1 \\
\hline Nicosia & Сyp & $\begin{array}{lll}1.5 & 2.6 & 4.6\end{array}$ & 0.7 & 1 & 18 & 79 & 0 & 0 & 0 & 0 \\
\hline Oslo & Nor & $1.3 \quad 2.23 .6$ & 0.9 & 19 & 23 & 55 & 1 & 0 & 1 & 1 \\
\hline Paris & France & $\begin{array}{lll}1.0 & 2.0 & 3.8\end{array}$ & 1.0 & 19 & 17 & 56 & 3 & 1 & 1 & 2 \\
\hline Prague & Czech Republic & $\begin{array}{lll}1.4 & 2.3 & 3.9\end{array}$ & 1.2 & 26 & 26 & 41 & 3 & 1 & 1 & 2 \\
\hline Rabat & Morocco & $\begin{array}{lll}0.8 & 1.7 & 2.9\end{array}$ & 0.5 & 6 & 10 & 76 & 2 & 1 & 1 & 2 \\
\hline Riga & Latvia & $\begin{array}{lll}1.5 & 2.3 & 3.8\end{array}$ & 1.0 & 17 & 28 & 48 & 2 & 1 & 1 & 2 \\
\hline Rome & Italy & $\begin{array}{lll}1.4 & 2.7 & 4.7\end{array}$ & 0.9 & 8 & 16 & 69 & 2 & 0 & 0 & 1 \\
\hline Saint Helier & Jersey & $\begin{array}{lll}0.9 & 1.7 & 3.0\end{array}$ & 0.6 & 7 & 26 & 64 & 1 & 0 & 1 & 0 \\
\hline Saint Peter Port & Guernsey and A. & $\begin{array}{llll}0.8 & 1.6 & 2.8\end{array}$ & 0.6 & 1 & 27 & 69 & 0 & 0 & 1 & 0 \\
\hline San Marino & San Marino & $\begin{array}{lll}1.4 & 2.8 & 4.9\end{array}$ & 1.3 & 16 & 22 & 56 & 3 & 1 & 0 & 1 \\
\hline Sarajevo & Bosnia and $\mathrm{H}$. & $\begin{array}{lll}1.6 & 2.8 & 5.0\end{array}$ & 1.4 & 19 & 25 & 51 & 2 & 0 & 0 & 1 \\
\hline Skopje & Macedonia & $\begin{array}{lll}1.7 & 3.1 & 5.5\end{array}$ & 1.3 & 11 & 25 & 60 & 1 & 0 & 0 & 1 \\
\hline Sofia & Bulgaria & $\begin{array}{lll}1.7 & 3.1 & 5.4\end{array}$ & 1.3 & 12 & 25 & 59 & 1 & 0 & 0 & 1 \\
\hline Stockholm & Sweden & $\begin{array}{lll}1.4 & 2.3 & 3.7\end{array}$ & 0.9 & 16 & 26 & 52 & 2 & 0 & 1 & 1 \\
\hline Tallinn & Estonia & $\begin{array}{lll}1.6 & 2.5 & 3.9\end{array}$ & 0.9 & 12 & 30 & 51 & 1 & 1 & 1 & 1 \\
\hline Tirana & Albania & $\begin{array}{lll}1.6 & 3.0 & 5.3\end{array}$ & 1.1 & 12 & 18 & 64 & 2 & 1 & 0 & 1 \\
\hline Torshavn & Faroe Islands & $\begin{array}{lll}0.5 & 1.4 & 2.2\end{array}$ & 0.6 & 2 & 34 & 59 & 1 & 0 & 1 & 1 \\
\hline Tripoli & Libya & $\begin{array}{lll}1.5 & 2.5 & 4.5\end{array}$ & 0.8 & 2 & 22 & 72 & 1 & 0 & 0 & 1 \\
\hline Tunis & Tunisia & $\begin{array}{lll}1.4 & 2.5 & 4.4\end{array}$ & 0.8 & 4 & 20 & 73 & 1 & 0 & 0 & 1 \\
\hline Vaduz & Liechtenstein & $\begin{array}{lll}1.4 & 2.5 & 4.4\end{array}$ & 1.2 & 19 & 22 & 51 & 4 & 1 & 1 & 2 \\
\hline Valletta & Malta & $\begin{array}{lll}1.5 & 2.5 & 4.3\end{array}$ & 0.7 & 4 & 23 & 71 & 1 & 0 & 0 & 0 \\
\hline Vatican City & Vatican City & $1.4 \quad 2.7 \quad 4.7$ & 0.9 & 8 & 16 & 69 & 2 & 0 & 0 & 1 \\
\hline Vienna & Austria & 1.42 .44 .2 & 1.3 & 25 & 25 & 44 & 3 & 1 & 1 & 2 \\
\hline Vilnius & Lithuania & $1.6 \quad 2.44 .0$ & 1.2 & 30 & 24 & 39 & 2 & 1 & 1 & 2 \\
\hline Warsaw & Poland & $\begin{array}{llll}1.5 & 2.4 & 3.9\end{array}$ & 1.3 & 31 & 24 & 38 & 2 & 1 & 1 & 2 \\
\hline Zagreb & Croatia & $\begin{array}{lll}1.5 & 2.7 & 4.8\end{array}$ & 1.4 & 23 & 20 & 49 & 3 & 11 & 1 & 2 \\
\hline
\end{tabular}


Table S16. Relative changes of total precipitation in summer (JJA) in the near future (2021-2050) compared to the period 1981-2010 (see legend of Table S10 for further details).

\begin{tabular}{|c|c|c|c|c|c|c|c|c|c|c|c|c|c|}
\hline \multirow{3}{*}{ Capital city } & \multirow{3}{*}{ Country } & \multicolumn{4}{|c|}{$B M_{k} \& B U(\%)$} & \multicolumn{8}{|c|}{ \% Total variance } \\
\hline & & \multicolumn{3}{|c|}{$\mathrm{RCP}$} & \multirow{2}{*}{$B U$} & \multicolumn{3}{|c|}{ Main effects } & \multicolumn{3}{|c|}{ Inter. } & \multirow[b]{2}{*}{ IV } & \multirow[b]{2}{*}{ RV } \\
\hline & & 2.6 & 4.5 & 8.5 & & $\mathrm{RCM}$ & GCM & $\mathrm{RCP}$ & & & I3 & & \\
\hline Amman & Jordan & -16 & -18 & -13 & 43 & 11 & 23 & 0 & 9 & 1 & 3 & 49 & 6 \\
\hline Algiers & Algeria & 2 & 3 & -4 & 21 & 8 & 33 & 2 & 13 & 3 & 5 & 30 & 6 \\
\hline Amsterdam & Netherlands & -1 & -2 & -2 & 8 & 31 & 17 & 0 & 14 & 3 & 3 & 22 & 9 \\
\hline Andorra la Vella & Andorra & -1 & -7 & -8 & 8 & 12 & 33 & 12 & 9 & 4 & 6 & 19 & 6 \\
\hline Ankara & Turkey & -8 & -12 & -13 & 14 & 17 & 23 & 3 & 13 & 2 & 7 & 29 & 6 \\
\hline Athens & Greece & -3 & -6 & -17 & 19 & 15 & 17 & 9 & 12 & 3 & 7 & 32 & 6 \\
\hline Bayrut & Lebanon & -4 & -7 & -13 & 38 & 4 & 22 & 1 & 14 & 1 & 6 & 41 & 11 \\
\hline Belgrade & Serbia and $\mathrm{M}$. & -1 & -5 & -7 & 10 & 21 & 26 & 5 & 10 & 1 & 5 & 25 & 7 \\
\hline Berlin & Germany & -0 & 0 & 1 & 9 & 28 & 25 & 0 & 6 & 3 & 3 & 22 & 13 \\
\hline Bern & Switzerland & -1 & -3 & -5 & 8 & 25 & 41 & 3 & 11 & 2 & 2 & 13 & 3 \\
\hline Bratislava & Slovakia & -0 & -2 & 0 & 8 & 17 & 29 & 1 & 8 & 2 & 4 & 31 & 8 \\
\hline Brussels & Belgium & -4 & -5 & -6 & 8 & 32 & 29 & 1 & 11 & 2 & 1 & 17 & 5 \\
\hline Bucharest & Romania & -2 & -6 & -7 & 12 & 6 & 41 & 4 & 11 & 1 & 4 & 24 & 7 \\
\hline Budapest & Hungary & -1 & -3 & -2 & 9 & 19 & 27 & 1 & 11 & 3 & 3 & 30 & 7 \\
\hline Cairo & Egypt & -17 & -8 & -20 & 108 & 7 & 13 & 0 & 13 & 1 & 8 & 44 & 13 \\
\hline Chisinau & Moldova & -1 & -5 & -6 & 9 & 14 & 30 & 5 & 7 & 3 & 3 & 33 & 4 \\
\hline Copenhagen & Denmark & -1 & 1 & 1 & 9 & 26 & 36 & 1 & 6 & 1 & 4 & 20 & 5 \\
\hline Damascus & Syria & 2 & -24 & 0 & 139 & 7 & 3 & 1 & 9 & 3 & 4 & 55 & 18 \\
\hline Douglas & Isle of Man & -2 & -2 & -5 & 7 & 40 & 16 & 4 & 10 & 2 & 5 & 17 & 5 \\
\hline Dublin & Ireland & -4 & -3 & -6 & 6 & 20 & 22 & 4 & 10 & 1 & 8 & 27 & 8 \\
\hline Gibraltar & Gibraltar & 5 & -3 & -9 & 21 & 26 & 14 & 7 & 7 & 3 & 5 & 33 & 6 \\
\hline Helsinki & Finland & 1 & 6 & 7 & 9 & 24 & 30 & 7 & 5 & 1 & 6 & 21 & 6 \\
\hline Jerusalem & Israel & -18 & -19 & -13 & 42 & 17 & 19 & 0 & 9 & 1 & 7 & 39 & 7 \\
\hline Kiev & Ukraine & -1 & -2 & -2 & 11 & 19 & 37 & 0 & 13 & 1 & 2 & 24 & 4 \\
\hline Lisbon & Portugal & -6 & -18 & -24 & 14 & 10 & 12 & 22 & 6 & 4 & 8 & 33 & 5 \\
\hline Ljubljana & Slovenia & -2 & -5 & -5 & 9 & 25 & 33 & 2 & 7 & 1 & 4 & 22 & 7 \\
\hline London & UK & -1 & -3 & -4 & 8 & 32 & 23 & 3 & 10 & 1 & 5 & 22 & 5 \\
\hline Luxemburg & Luxembourg & -4 & -4 & -6 & 7 & 22 & 30 & 1 & 12 & 2 & 4 & 22 & 7 \\
\hline Madrid & Spain & -2 & -7 & -12 & 14 & 17 & 40 & 8 & 6 & 1 & 2 & 22 & 4 \\
\hline Minsk & Belarus & -3 & 1 & 1 & 9 & 12 & 35 & 3 & 13 & 1 & 3 & 23 & 9 \\
\hline Monaco-Ville & Monaco & -0 & -8 & -8 & 14 & 19 & 31 & 7 & 10 & 2 & 5 & 21 & 5 \\
\hline Nicosia & Cypr & -6 & -21 & -11 & 44 & 4 & 20 & 2 & 9 & 1 & 3 & 49 & 11 \\
\hline Oslo & Norw & -1 & 1 & 1 & 9 & 43 & 25 & 1 & 4 & 1 & 3 & 19 & 4 \\
\hline Paris & France & -4 & -5 & -7 & 8 & 36 & 20 & 2 & 9 & 3 & 2 & 21 & 7 \\
\hline Prague & Czech Republic & 1 & 1 & 2 & 8 & 19 & 27 & 0 & 12 & 2 & 3 & 26 & 12 \\
\hline Rabat & Morocco & 1 & 0 & -3 & 26 & 6 & 42 & 0 & 5 & 0 & 3 & 41 & 3 \\
\hline Riga & Latvia & 2 & 4 & 5 & 9 & 26 & 32 & 2 & 7 & 1 & 4 & 20 & 8 \\
\hline Rome & Italy & -0 & -10 & -12 & 15 & 20 & 30 & 10 & 9 & 1 & 6 & 18 & 5 \\
\hline Saint Helier & Jersey & -2 & -4 & -6 & 8 & 26 & 14 & 5 & 17 & 2 & 4 & 24 & 8 \\
\hline Saint Peter Port & Guernsey and A. & -1 & -3 & -6 & 8 & 30 & 12 & 6 & 13 & 3 & 5 & 25 & 7 \\
\hline San Marino & San Marino & 1 & -5 & -6 & 14 & 17 & 39 & 4 & 11 & 2 & 6 & 16 & 6 \\
\hline Sarajevo & Bosnia and $\mathrm{H}$. & -2 & -7 & -9 & 10 & 22 & 25 & 7 & 11 & 1 & 5 & 22 & 6 \\
\hline Skopje & Macedonia & -4 & -9 & -11 & 14 & 22 & 21 & 5 & 10 & 1 & 4 & 28 & 10 \\
\hline Sofia & Bulgaria & -4 & -8 & -11 & 10 & 13 & 34 & 8 & 9 & 1 & 6 & 21 & 8 \\
\hline Stockholm & Sweden & 1 & 2 & 3 & 9 & 27 & 32 & 1 & 7 & 1 & 6 & 20 & 6 \\
\hline Tallinn & Estonia & 1 & 5 & 6 & 9 & 23 & 31 & 6 & 8 & 1 & 4 & 19 & 8 \\
\hline Tirana & Albania & -2 & -11 & -13 & 13 & 16 & 25 & 11 & 15 & 3 & 8 & 18 & 5 \\
\hline Torshavn & Faroe Islands & -0 & 1 & -1 & 6 & 36 & 28 & 3 & 7 & 2 & 2 & 18 & 3 \\
\hline Tripoli & Libya & 7 & -4 & -13 & 27 & 17 & 6 & 9 & 14 & 6 & 3 & 38 & 8 \\
\hline Tunis & Tunisia & -3 & -13 & -16 & 19 & 19 & 28 & 8 & 10 & 2 & 5 & 25 & 3 \\
\hline Vaduz & Liechtenstein & -2 & -3 & -3 & 8 & 23 & 40 & 1 & 13 & 2 & 3 & 14 & 5 \\
\hline Valletta & Malta & 2 & -9 & -12 & 24 & 12 & 20 & 6 & 12 & 2 & 3 & 41 & 5 \\
\hline Vatican City & Vatican City & -0 & -10 & -12 & 15 & 20 & 30 & 10 & 9 & 1 & 6 & 18 & 5 \\
\hline Vienna & Austria & -1 & 0 & 1 & 8 & 13 & 35 & 1 & 10 & 3 & 2 & 32 & 5 \\
\hline Vilnius & Lithuania & -1 & 0 & 1 & 8 & 20 & 32 & 1 & 12 & 2 & 4 & 20 & 8 \\
\hline Warsaw & Poland & 0 & 1 & 2 & 9 & 27 & 32 & 1 & 9 & 1 & 2 & 20 & 8 \\
\hline Zagreb & Croatia & -3 & -5 & -6 & 9 & 20 & 34 & 2 & 8 & 1 & 4 & 26 & 5 \\
\hline
\end{tabular}


Table S17. Relative changes of total precipitation in summer (JJA) at the end of the century (2071-2099) compared to the period 1981-2010 (see legend of Table S10 for further details).

\begin{tabular}{|c|c|c|c|c|c|c|c|c|c|c|c|c|c|}
\hline \multirow{3}{*}{ Capital city } & \multirow[b]{3}{*}{ Country } & \multicolumn{4}{|c|}{$B M_{k} \& B U(\%)$} & \multicolumn{8}{|c|}{ \% Total variance } \\
\hline & & \multicolumn{3}{|c|}{ RCP } & \multirow{2}{*}{$B U$} & \multicolumn{3}{|c|}{ Main effects } & \multicolumn{3}{|c|}{ Inter. } & \multirow[b]{2}{*}{ IV } & \multirow[b]{2}{*}{ RV } \\
\hline & & 2.6 & 4.5 & 8.5 & & $\mathrm{RCM}$ & GCM & $\mathrm{RCP}$ & I1 & $\mathrm{I} 2$ & $\mathrm{I} 3$ & & \\
\hline Amman & Jordan & -24 & -5 & -24 & 85 & 6 & 33 & 1 & 25 & 2 & 5 & 12 & 15 \\
\hline Algiers & Algeria & 5 & 8 & -16 & 40 & 10 & 43 & 7 & 11 & 4 & 10 & 8 & 7 \\
\hline Amsterdam & Netherlands & -1 & -4 & -9 & 16 & 44 & 17 & 3 & 10 & 3 & 5 & 5 & 13 \\
\hline Andorra la Vella & Andorra & -5 & -13 & -25 & 16 & 16 & 35 & 20 & 8 & 4 & 6 & 4 & 6 \\
\hline Ankara & Turkey & -14 & -20 & -33 & 25 & 10 & 37 & 9 & 14 & 3 & 10 & 8 & 9 \\
\hline Athens & Greece & 2 & -8 & -40 & 31 & 9 & 15 & 25 & 7 & 10 & 11 & 10 & 13 \\
\hline Bayrut & Lebanon & -24 & -3 & -32 & 67 & 8 & 35 & 3 & 20 & 3 & 6 & 13 & 12 \\
\hline Belgrade & Serbia and M. & -1 & -9 & -17 & 18 & 26 & 23 & 11 & 11 & 3 & 7 & 7 & 10 \\
\hline Berlin & Germany & -1 & 0 & 1 & 19 & 31 & 37 & 0 & 6 & 2 & 6 & 5 & 13 \\
\hline Bern & Switzerland & -0 & -5 & -12 & 17 & 30 & 39 & 8 & 11 & 2 & 4 & 3 & 4 \\
\hline Bratislava & Slovakia & 2 & -3 & -1 & 17 & 21 & 39 & 1 & 13 & 2 & 6 & 8 & 10 \\
\hline Brussels & Belgium & -4 & -10 & -14 & 18 & 39 & 30 & 5 & 9 & 3 & 2 & 4 & 8 \\
\hline Bucharest & Romania & -2 & -11 & -17 & 20 & 12 & 38 & 9 & 11 & 3 & 6 & 8 & 13 \\
\hline Budapest & Hungary & 0 & -5 & -7 & 18 & 20 & 31 & 3 & 14 & 3 & 9 & 8 & 13 \\
\hline Cairo & Egypt & -9 & -1 & -35 & 242 & 9 & 19 & 0 & 19 & 3 & 16 & 9 & 25 \\
\hline Chisinau & Moldova & -4 & -8 & -15 & 18 & 13 & 46 & 6 & 8 & 4 & 4 & 9 & 9 \\
\hline Copenhagen & Denmark & -2 & 2 & 1 & 19 & 29 & 42 & 0 & 9 & 2 & 5 & 5 & 8 \\
\hline Damascus & Syria & 35 & 2 & 44 & 392 & 14 & 6 & 0 & 16 & 7 & 7 & 7 & 43 \\
\hline Douglas & Isle of Man & -1 & -3 & -12 & 14 & 45 & 12 & 10 & 10 & 5 & 6 & 5 & 7 \\
\hline Dublin & Irelar & -6 & -8 & -14 & 11 & 23 & 25 & 9 & 13 & 2 & 10 & 7 & 11 \\
\hline Gibraltar & Gibral & 6 & -16 & -30 & 37 & 19 & 22 & 14 & 6 & 6 & 13 & 10 & 9 \\
\hline Helsinki & Finland & 1 & 11 & 15 & 19 & 31 & 34 & 9 & 4 & 1 & 7 & 5 & 8 \\
\hline Jerusalem & Israel & -33 & -12 & -20 & 73 & 16 & 37 & 1 & 8 & 2 & 10 & 13 & 12 \\
\hline Kiev & Ukraine & -2 & -6 & -6 & 20 & 25 & 41 & 1 & 15 & 2 & 4 & 7 & 5 \\
\hline Lisbon & Portugal & -11 & -36 & -58 & 22 & 11 & 17 & 43 & 4 & 3 & 6 & 10 & 7 \\
\hline Ljubljana & Slovenia & -3 & -8 & -16 & 19 & 35 & 29 & 8 & 9 & 2 & 6 & 5 & 8 \\
\hline London & UK & -1 & -8 & -12 & 15 & 42 & 22 & 9 & 8 & 2 & 5 & 6 & 7 \\
\hline Luxemburg & Luxembourg & -8 & -11 & -16 & 16 & 29 & 40 & 3 & 8 & 4 & 4 & 4 & 7 \\
\hline Madrid & Spain & -6 & -16 & -34 & 25 & 18 & 44 & 18 & 7 & 1 & 2 & 7 & 4 \\
\hline Minsk & Belar & -6 & -1 & -1 & 18 & 24 & 40 & 2 & 13 & 2 & 4 & 6 & 9 \\
\hline Monaco-Ville & Mon & 4 & -17 & -24 & 27 & 26 & 23 & 17 & 11 & 2 & 7 & 5 & 8 \\
\hline Nicc & & -7 & -40 & -24 & 101 & 8 & 19 & 2 & 14 & 6 & 8 & 9 & 34 \\
\hline Oslo & Nor & -2 & 1 & 2 & 18 & 49 & 30 & 1 & 5 & 2 & 4 & 4 & 6 \\
\hline Paris & France & -6 & -12 & -19 & 17 & 39 & 25 & 9 & 8 & 4 & 3 & 4 & 8 \\
\hline Prague & Czech Republic & 1 & 1 & 1 & 15 & 26 & 35 & 0 & 12 & 2 & 5 & 6 & 14 \\
\hline Rabat & Morocco & 2 & 1 & -17 & 38 & 10 & 41 & 5 & 4 & 4 & 8 & 19 & 10 \\
\hline Riga & Latvia & 1 & 5 & 8 & 18 & 28 & 45 & 3 & 7 & 1 & 4 & 5 & 8 \\
\hline Rome & Italy & 5 & -16 & -30 & 29 & 25 & 25 & 20 & 10 & 3 & 7 & 4 & 7 \\
\hline Saint Helier & Jersey & -1 & -9 & -20 & 17 & 30 & 20 & 16 & 12 & 2 & 5 & 5 & 10 \\
\hline Saint Peter Port & Guernsey and A. & 0 & -6 & -18 & 17 & 32 & 18 & 18 & 11 & 3 & 4 & 5 & 8 \\
\hline San Marino & San Marino & 4 & -6 & -17 & 26 & 23 & 31 & 9 & 13 & 4 & 7 & 4 & 9 \\
\hline Sarajevo & Bosnia and $\mathrm{H}$. & -3 & -13 & -22 & 19 & 28 & 23 & 14 & 12 & 2 & 6 & 5 & 9 \\
\hline Skop & acedonia & -6 & -13 & -25 & 23 & 30 & 17 & 10 & 13 & 2 & 5 & 10 & 12 \\
\hline Sofia & Bulgaria & -7 & -19 & -27 & 18 & 13 & 37 & 17 & 9 & 1 & 6 & 6 & 11 \\
\hline Stockholm & Sweden & 2 & 5 & 6 & 18 & 35 & 35 & 1 & 7 & 1 & 6 & 5 & 9 \\
\hline Tallinn & Estonia & -2 & 6 & 12 & 21 & 27 & 40 & 6 & 7 & 1 & 5 & 4 & 10 \\
\hline Tirana & Albania & -1 & -21 & -34 & 25 & 20 & 18 & 22 & 15 & 3 & 10 & 4 & 7 \\
\hline Torshavn & Faroe Islands & -2 & 2 & -3 & 12 & 42 & 33 & 4 & 6 & 2 & 3 & 5 & 5 \\
\hline Tripoli & Libya & 17 & -14 & -44 & 44 & 19 & 4 & 24 & 14 & 10 & 6 & 12 & 11 \\
\hline Tunis & Tunisia & -6 & -22 & -40 & 33 & 21 & 26 & 14 & 12 & 2 & 10 & 8 & 7 \\
\hline Vaduz & Liechtenstein & -3 & -5 & -10 & 17 & 28 & 43 & 3 & 12 & 3 & 3 & 3 & 5 \\
\hline Valletta & Malta & 10 & -16 & -30 & 39 & 13 & 19 & 16 & 14 & 6 & 6 & 13 & 14 \\
\hline Vatican City & Vatican City & 5 & -16 & -30 & 29 & 25 & 25 & 20 & 10 & 3 & 7 & 4 & 7 \\
\hline Vienna & Austria & 1 & 0 & -1 & 15 & 19 & 44 & 0 & 12 & 4 & 3 & 9 & 9 \\
\hline Vilnius & Lithuania & -5 & -1 & 0 & 18 & 27 & 43 & 1 & 10 & 2 & 5 & 5 & 7 \\
\hline Warsaw & Poland & 2 & 0 & 2 & 20 & 30 & 43 & 0 & 10 & 1 & 3 & 4 & 9 \\
\hline Zagreb & Croatia & -4 & -8 & -16 & 17 & 30 & 34 & 7 & 7 & 2 & 7 & 6 & 6 \\
\hline
\end{tabular}




\section{Differences between "balanced" and direct estimates of mean projected changes}

Table S18. Difference between "balanced" estimates $B M_{k}$ of mean expected change response (in ${ }^{\circ} \mathrm{C}$ ), and corresponding direct estimates $M_{k}$ for the three regions taken from the IPCC SREX report (Seneviratne et al., 2012), for absolute changes of mean temperature in winter (DJF) and summer (JJA) at the end of the century (2071-2099) compared to the period 1981-2010.

\begin{tabular}{|c|rrr|rrr|}
\hline \hline Season & \multicolumn{3}{|c|}{ DJF } & \multicolumn{3}{|c|}{ JJA } \\
\hline Country & \multicolumn{2}{|c|}{$\mathrm{BM}_{k}-\mathrm{M}_{k}$} & \multicolumn{2}{c|}{$\mathrm{BM}_{k}-\mathrm{M}_{k}$} \\
& 2.6 & 4.5 & 8.5 & 2.6 & 4.5 & 8.5 \\
\hline NEU & -0.1 & -0.2 & -0.3 & 0.1 & 0.1 & -0.1 \\
CEU & -0.2 & -0.2 & -0.4 & 0.5 & 0.5 & 0.2 \\
MED & 0.1 & -0.1 & -0.2 & 0.2 & 0.3 & -0.0 \\
\hline
\end{tabular}

Table S19. Difference between "balanced" estimates $B M_{k}$ of mean expected change response (in \%), and corresponding direct estimates $M_{k}$ for the three regions taken from the IPCC SREX report (Seneviratne et al., 2012), for relative changes of total precipitation in winter (DJF) and summer (JJA) at the end of the century (2071-2099) compared to the period 1981-2010.

\begin{tabular}{|c|rrr|rrr|}
\hline \hline Season & \multicolumn{2}{|c|}{ DJF } & \multicolumn{3}{|c|}{ JJA } \\
\hline \multirow{2}{*}{ Country } & \multicolumn{2}{|c|}{$\mathrm{BM}_{k}-\mathrm{M}_{k}$} & \multicolumn{2}{|c|}{$\mathrm{BM}_{k}-\mathrm{M}_{k}$} \\
& 2.6 & 4.5 & 8.5 & 2.6 & 4.5 & 8.5 \\
\hline NEU & -0 & -2 & -2 & -2 & -4 & -3 \\
CEU & 0 & -2 & -2 & -4 & -9 & -4 \\
MED & -0 & -3 & 1 & -4 & -7 & -3 \\
\hline
\end{tabular}


Table S20. Difference between "balanced" estimates $B M_{k}$ of mean expected change response (in ${ }^{\circ} \mathrm{C}$ ), and corresponding direct estimates $M_{k}$ for the countries of the domain, for absolute changes of mean temperature in winter (DJF) and summer (JJA) at the end of the century (2071-2099) compared to the period 1981-2010.

\begin{tabular}{|c|c|c|c|c|c|c|}
\hline \multirow[b]{3}{*}{ Country } & \multicolumn{3}{|c|}{ DJF } & \multicolumn{3}{|c|}{ JJA } \\
\hline & \multicolumn{3}{|c|}{$\mathrm{BM}_{k}-\mathrm{M}_{k}$} & \multicolumn{3}{|c|}{$\mathrm{BM}_{k}-\mathrm{M}_{k}$} \\
\hline & 2.6 & 4.5 & 8.5 & 2.6 & 4.5 & 8.5 \\
\hline Algeria & 0.1 & -0.1 & -0.2 & 0.2 & 0.1 & -0.0 \\
\hline Albania & 0.1 & -0.1 & -0.2 & 0.3 & 0.4 & 0.1 \\
\hline Bosnia and $\mathrm{H}$. & 0.0 & -0.1 & -0.3 & 0.5 & 0.6 & 0.2 \\
\hline Bulgaria & 0.1 & -0.1 & -0.2 & 0.5 & 0.5 & 0.2 \\
\hline Cyprus & 0.1 & -0.1 & -0.1 & 0.1 & 0.1 & -0.1 \\
\hline Denmark & -0.1 & -0.2 & -0.4 & 0.2 & 0.3 & -0.1 \\
\hline Ireland & -0.1 & -0.3 & -0.3 & 0.1 & 0.0 & -0.1 \\
\hline Estonia & -0.4 & -0.2 & -0.5 & 0.4 & 0.3 & 0.0 \\
\hline Austria & -0.1 & -0.2 & -0.4 & 0.4 & 0.5 & 0.1 \\
\hline Czech Rep. & -0.1 & -0.2 & -0.4 & 0.4 & 0.6 & 0.2 \\
\hline Finland & -0.2 & -0.1 & -0.3 & 0.3 & 0.3 & -0.0 \\
\hline France & -0.1 & -0.2 & -0.4 & 0.2 & 0.3 & 0.0 \\
\hline Germany & -0.1 & -0.2 & -0.5 & 0.3 & 0.4 & 0.1 \\
\hline Greece & 0.1 & -0.1 & -0.2 & 0.3 & 0.4 & 0.1 \\
\hline Croatia & 0.0 & -0.1 & -0.3 & 0.5 & 0.6 & 0.2 \\
\hline Hungary & -0.0 & -0.1 & -0.3 & 0.6 & 0.7 & 0.2 \\
\hline Israel & 0.2 & -0.2 & -0.1 & 0.3 & 0.3 & -0.0 \\
\hline Italy & 0.0 & -0.1 & -0.3 & 0.3 & 0.4 & 0.1 \\
\hline Jordan & 0.2 & -0.1 & -0.1 & 0.3 & 0.3 & -0.0 \\
\hline Lebanon & 0.2 & -0.2 & -0.1 & 0.3 & 0.3 & -0.0 \\
\hline Latvia & -0.4 & -0.2 & -0.5 & 0.5 & 0.4 & 0.1 \\
\hline Belarus & -0.4 & -0.2 & -0.5 & 0.6 & 0.6 & 0.2 \\
\hline Lithuania & -0.4 & -0.2 & -0.5 & 0.5 & 0.4 & 0.1 \\
\hline Slovakia & -0.1 & -0.3 & -0.4 & 0.6 & 0.7 & 0.3 \\
\hline Macedonia & 0.1 & -0.2 & -0.2 & 0.4 & 0.5 & 0.2 \\
\hline Morocco & -0.0 & -0.2 & -0.2 & 0.1 & 0.1 & -0.2 \\
\hline Netherlands & -0.1 & -0.3 & -0.4 & 0.2 & 0.2 & -0.1 \\
\hline Norway & -0.1 & -0.2 & -0.3 & 0.3 & 0.2 & -0.1 \\
\hline Poland & -0.3 & -0.3 & -0.5 & 0.5 & 0.6 & 0.2 \\
\hline Portugal & -0.0 & -0.1 & -0.3 & -0.1 & 0.2 & -0.1 \\
\hline Romania & -0.0 & -0.1 & -0.3 & 0.6 & 0.7 & 0.3 \\
\hline Moldova & -0.1 & -0.1 & -0.3 & 0.6 & 0.7 & 0.3 \\
\hline Slovenia & -0.1 & -0.2 & -0.4 & 0.5 & 0.6 & 0.2 \\
\hline Spain & -0.0 & -0.1 & -0.3 & 0.1 & 0.2 & -0.1 \\
\hline Sweden & -0.3 & -0.3 & -0.4 & 0.3 & 0.2 & -0.0 \\
\hline Switzerland & -0.1 & -0.2 & -0.4 & 0.3 & 0.3 & 0.1 \\
\hline Tunisia & 0.0 & -0.1 & -0.2 & 0.2 & 0.1 & -0.0 \\
\hline Turkey & 0.2 & -0.2 & -0.1 & 0.3 & 0.4 & 0.1 \\
\hline UK & -0.1 & -0.3 & -0.4 & 0.1 & 0.1 & -0.1 \\
\hline Ukraine & -0.1 & -0.1 & -0.3 & 0.6 & 0.6 & 0.3 \\
\hline
\end{tabular}


Table S21. Difference between "balanced" estimates $B M_{k}$ of mean expected change response (in \%), and corresponding direct estimates $M_{k}$ for the countries of the domain, for relative changes of total precipitation in winter (DJF) and summer (JJA) at the end of the century (2071-2099) compared to the period 1981-2010.

\begin{tabular}{|c|rrr|rrr|}
\hline \hline Season & \multicolumn{3}{|c|}{ DJF } & \multicolumn{3}{|c|}{ JJA } \\
\hline Country & \multicolumn{3}{|c|}{$\mathrm{BM}_{k}-\mathrm{M}_{k}$} & \multicolumn{2}{|c|}{$\mathrm{BM}_{k}-\mathrm{M}_{k}$} \\
\hline Algeria & 2.6 & 4.5 & 8.5 & 2.6 & 4.5 & 8.5 \\
Albania & 2 & 0 & 3 & -9 & -4 & -2 \\
Bosnia and H. & 1 & -0 & 3 & -6 & -6 & -3 \\
Bulgaria & 3 & -1 & 4 & -4 & -8 & -6 \\
Cyprus & -5 & -5 & -1 & -4 & -16 & -8 \\
Denmark & -1 & -2 & -3 & -1 & -6 & -5 \\
Ireland & -3 & -3 & -2 & -1 & -5 & -4 \\
Estonia & -2 & -2 & -3 & -5 & -9 & -5 \\
Austria & -1 & -4 & -2 & -1 & -5 & 0 \\
Czech Rep. & -1 & -2 & -2 & -2 & -6 & -2 \\
Finland & 1 & -3 & -2 & -2 & -4 & -3 \\
France & 1 & -2 & -0 & -3 & -6 & -4 \\
Germany & 1 & -1 & -2 & -3 & -8 & -4 \\
Greece & 2 & -0 & 5 & -4 & -4 & -5 \\
Croatia & 1 & -0 & 2 & -3 & -8 & -2 \\
Hungary & -0 & -3 & -1 & -3 & -11 & -2 \\
Israel & -9 & -2 & 0 & -10 & -39 & -13 \\
Italy & 1 & -5 & 3 & -1 & -7 & -2 \\
Jordan & -6 & -1 & 2 & -24 & 11 & -15 \\
Lebanon & -8 & -3 & -0 & -7 & -15 & -7 \\
Latvia & -1 & -2 & -3 & -4 & -9 & -6 \\
Belarus & -1 & -2 & -3 & -7 & -13 & -6 \\
Lithuania & -1 & -1 & -3 & -5 & -10 & -7 \\
Slovakia & -1 & -4 & -1 & -4 & -10 & -3 \\
Macedonia & 3 & -1 & 5 & -4 & -5 & -3 \\
Morocco & -1 & -5 & 2 & -5 & 7 & 4 \\
Netherlands & 0 & -2 & -2 & -3 & -8 & -5 \\
Norway & -2 & -4 & -4 & -2 & -3 & -1 \\
Poland & -2 & -2 & -3 & -3 & -10 & -6 \\
Portugal & -1 & -8 & -4 & -1 & -6 & -1 \\
Romania & 1 & -1 & 1 & -4 & -10 & -5 \\
Moldova & -2 & -7 & 1 & -5 & -13 & -6 \\
Slovenia & 1 & -3 & 1 & -2 & -8 & -1 \\
Spain & -1 & -8 & -3 & -4 & -5 & -0 \\
Sweden & -1 & -5 & -3 & -0 & -4 & -2 \\
Switzerland & 1 & -1 & -1 & -1 & -7 & -3 \\
Tunisia & -1 & -4 & 2 & -7 & -10 & -6 \\
Turkey & -1 & -3 & 0 & -4 & -9 & -4 \\
Ukraine & -2 & -3 & -2 & 0 & -4 & -3 \\
& -1 & -3 & -1 & -6 & -13 & -6 \\
\hline
\end{tabular}


Table S22. Difference between "balanced" estimates $B M_{k}$ of mean expected change response (in ${ }^{\circ} \mathrm{C}$ ), and corresponding direct estimates $M_{k}$ for the capital cities of the domain, for absolute changes of mean temperature in winter (DJF) and summer (JJA) at the end of the century (2071-2099) compared to the period 1981-2010.

\begin{tabular}{|c|c|c|c|c|c|c|c|}
\hline \multicolumn{2}{|c|}{ Season } & \multicolumn{3}{|c|}{ DJF } & \multicolumn{3}{|c|}{ JJA } \\
\hline \multirow{2}{*}{ Capital city } & \multirow[b]{2}{*}{ Country } & & $\overline{\mathrm{M}_{k}-\mathrm{N}}$ & & & $\overline{\mathrm{M}_{k}-\mathrm{N}}$ & \\
\hline & & 2.6 & 4.5 & 8.5 & 2.6 & 4.5 & 8.5 \\
\hline Amman & Jordan & 0.3 & -0.1 & -0.0 & 0.3 & 0.4 & 0.1 \\
\hline Algiers & Algeria & 0.0 & -0.1 & -0.2 & 0.2 & 0.2 & -0.1 \\
\hline Amsterdam & Netherlands & -0.1 & -0.3 & -0.4 & 0.2 & 0.2 & -0.0 \\
\hline Andorra la Vella & Andorra & -0.3 & -0.4 & -0.5 & 0.2 & 0.2 & 0.1 \\
\hline Ankara & Turkey & 0.1 & -0.3 & -0.1 & 0.5 & 0.6 & 0.3 \\
\hline Athens & Greece & 0.1 & -0.1 & -0.1 & 0.4 & 0.3 & 0.1 \\
\hline Bayrut & Lebanon & 0.1 & -0.1 & -0.1 & 0.1 & 0.2 & -0.1 \\
\hline Belgrade & Serbia and M. & 0.1 & -0.0 & -0.2 & 0.6 & 0.7 & 0.4 \\
\hline Berlin & Germany & -0.2 & -0.2 & -0.4 & 0.4 & 0.5 & 0.1 \\
\hline Bern & Switzerland & -0.0 & -0.2 & -0.4 & 0.3 & 0.4 & 0.2 \\
\hline Bratislava & Slovakia & -0.0 & -0.1 & -0.3 & 0.5 & 0.7 & 0.2 \\
\hline Brussels & Belgium & -0.1 & -0.3 & -0.4 & 0.2 & 0.3 & 0.1 \\
\hline Bucharest & Romania & 0.0 & -0.1 & -0.2 & 0.5 & 0.6 & 0.3 \\
\hline Budapest & Hungary & 0.0 & -0.1 & -0.3 & 0.6 & 0.7 & 0.3 \\
\hline Cairo & Egypt & 0.1 & -0.2 & -0.1 & 0.4 & 0.4 & 0.1 \\
\hline Chisinau & Moldova & -0.1 & -0.1 & -0.3 & 0.6 & 0.7 & 0.3 \\
\hline Copenhagen & Denmark & -0.1 & -0.1 & -0.3 & 0.2 & 0.3 & -0.0 \\
\hline Damascus & Syria & 0.2 & -0.1 & -0.0 & 0.2 & 0.3 & 0.0 \\
\hline Douglas & Isle of Man & -0.1 & -0.3 & -0.3 & 0.1 & 0.1 & -0.1 \\
\hline Dublin & Ireland & -0.1 & -0.3 & -0.3 & 0.0 & 0.1 & -0.1 \\
\hline Gibraltar & Gibraltar & -0.0 & -0.1 & -0.2 & 0.1 & 0.2 & -0.0 \\
\hline Helsinki & Finland & -0.4 & -0.1 & -0.4 & 0.3 & 0.3 & 0.0 \\
\hline Jerusalem & Israel & 0.2 & -0.1 & -0.0 & 0.3 & 0.4 & 0.1 \\
\hline Kiev & Ukraine & -0.2 & -0.2 & -0.3 & 0.6 & 0.6 & 0.3 \\
\hline Lisbon & Portugal & 0.0 & -0.1 & -0.2 & -0.0 & 0.1 & -0.1 \\
\hline Ljubljana & Slovenia & -0.0 & -0.1 & -0.3 & 0.5 & 0.6 & 0.3 \\
\hline London & UK & -0.1 & -0.3 & -0.4 & 0.1 & 0.2 & -0.0 \\
\hline Luxemburg & Luxembourg & -0.1 & -0.3 & -0.4 & 0.3 & 0.5 & 0.2 \\
\hline Madrid & Spain & 0.0 & -0.0 & -0.2 & 0.2 & 0.3 & 0.0 \\
\hline Minsk & Belarus & -0.3 & -0.2 & -0.4 & 0.7 & 0.6 & 0.3 \\
\hline Monaco-Ville & Monaco & 0.1 & -0.1 & -0.2 & 0.2 & 0.4 & 0.1 \\
\hline Nicosia & Cyprus & 0.1 & -0.1 & -0.1 & 0.1 & 0.2 & -0.1 \\
\hline Oslo & Norway & -0.3 & -0.4 & -0.5 & 0.3 & 0.3 & 0.1 \\
\hline Paris & France & -0.1 & -0.3 & -0.4 & 0.1 & 0.3 & 0.1 \\
\hline Prague & Czech Republic & -0.1 & -0.2 & -0.4 & 0.4 & 0.6 & 0.2 \\
\hline Rabat & Morocco & -0.0 & -0.2 & -0.2 & -0.0 & 0.1 & -0.1 \\
\hline Riga & Latvia & -0.5 & -0.2 & -0.4 & 0.4 & 0.3 & 0.0 \\
\hline Rome & Italy & 0.1 & 0.0 & -0.2 & 0.2 & 0.3 & 0.0 \\
\hline Saint Helier & Jersey & -0.1 & -0.3 & -0.3 & 0.1 & 0.1 & -0.1 \\
\hline Saint Peter Port & Guernsey and A. & -0.1 & -0.3 & -0.3 & 0.0 & 0.0 & -0.2 \\
\hline San Marino & San Marino & 0.0 & -0.1 & -0.3 & 0.4 & 0.5 & 0.2 \\
\hline Sarajevo & Bosnia and $\mathrm{H}$. & 0.0 & -0.2 & -0.2 & 0.5 & 0.5 & 0.2 \\
\hline Skopje & Macedonia & 0.0 & -0.2 & -0.2 & 0.4 & 0.6 & 0.2 \\
\hline Sofia & Bulgaria & 0.1 & -0.1 & -0.2 & 0.5 & 0.6 & 0.3 \\
\hline Stockholm & Sweden & -0.3 & -0.3 & -0.4 & 0.3 & 0.3 & 0.0 \\
\hline Tallinn & Estonia & -0.4 & -0.1 & -0.4 & 0.3 & 0.2 & -0.0 \\
\hline Tirana & Albania & 0.1 & -0.1 & -0.2 & 0.4 & 0.5 & 0.2 \\
\hline Torshavn & Faroe Islands & -0.2 & -0.3 & -0.3 & 0.0 & -0.0 & -0.2 \\
\hline Tripoli & Libya & 0.1 & -0.1 & -0.1 & 0.2 & 0.1 & 0.0 \\
\hline Tunis & Tunisia & 0.1 & -0.1 & -0.2 & 0.2 & 0.2 & -0.0 \\
\hline Vaduz & Liechtenstein & -0.1 & -0.2 & -0.4 & 0.3 & 0.4 & 0.2 \\
\hline Valletta & Malta & 0.1 & -0.0 & -0.1 & 0.3 & 0.3 & 0.0 \\
\hline Vatican City & Vatican City & 0.1 & 0.0 & -0.2 & 0.2 & 0.3 & 0.0 \\
\hline Vienna & Austria & -0.0 & -0.1 & -0.3 & 0.5 & 0.6 & 0.2 \\
\hline Vilnius & Lithuania & -0.4 & -0.2 & -0.4 & 0.6 & 0.6 & 0.2 \\
\hline Warsaw & Poland & -0.3 & -0.2 & -0.5 & 0.6 & 0.7 & 0.2 \\
\hline Zagreb & Croatia & 0.0 & -0.1 & -0.3 & 0.5 & 0.6 & 0.3 \\
\hline
\end{tabular}


Table S23. Difference between "balanced" estimates $B M_{k}$ of mean expected change response (in \%), and corresponding direct estimates $M_{k}$ for the capital cities of the domain, for relative changes of total precipitation in winter (DJF) and summer (JJA) at the end of the century (2071-2099) compared to the period 1981-2010.

\begin{tabular}{|c|c|c|c|c|c|c|c|}
\hline \multicolumn{2}{|c|}{ Season } & \multicolumn{3}{|c|}{ DJF } & \multicolumn{3}{|c|}{ JJA } \\
\hline Conital citt & Country & $\mathrm{BN}$ & $\Lambda_{k}-\mathrm{N}$ & & & $\mathbf{M}_{k^{-}}$ & \\
\hline Capital city & Country & 2.6 & 4.5 & 8.5 & 2.6 & 4.5 & 8.5 \\
\hline Amman & Jordan & \begin{tabular}{|l|}
-11 \\
\end{tabular} & -2 & -1 & -24 & -0 & -30 \\
\hline Algiers & Algeria & -0 & -3 & 4 & 0 & 8 & 8 \\
\hline Amsterdam & Netherlands & 0 & -1 & -1 & -2 & -6 & -5 \\
\hline Andorra la Vella & Andorra & 3 & -2 & 4 & -7 & -8 & -2 \\
\hline Ankara & Turkey & -0 & -2 & 1 & -5 & -8 & -3 \\
\hline Athens & Greece & 3 & 1 & 8 & -3 & -1 & -10 \\
\hline Bayrut & Lebanon & -7 & -2 & -0 & -15 & -38 & -12 \\
\hline Belgrade & Serbia and $\mathrm{M}$. & 1 & -1 & 2 & -6 & -10 & -7 \\
\hline Berlin & Germany & -0 & 0 & -2 & -5 & -9 & -8 \\
\hline Bern & Switzerland & 1 & 0 & -2 & 1 & -6 & -4 \\
\hline Bratislava & Slovakia & -1 & -4 & -0 & -5 & -11 & -3 \\
\hline Brussels & Belgium & 1 & -1 & -1 & -4 & -8 & -6 \\
\hline Bucharest & Romania & 4 & 1 & 6 & -6 & -12 & -8 \\
\hline Budapest & Hungary & -1 & -7 & -3 & -6 & -12 & -3 \\
\hline Cairo & Egypt & -8 & 5 & 0 & -9 & -12 & -157 \\
\hline Chisinau & Moldova & -2 & -7 & 0 & -8 & -13 & -7 \\
\hline Copenhagen & Denmark & 0 & -1 & -0 & -5 & -7 & -7 \\
\hline Damascus & Syria & -8 & -5 & -1 & 53 & -90 & 52 \\
\hline Douglas & Isle of Man & 1 & -1 & 1 & 1 & 1 & -3 \\
\hline Dublin & Ireland & -2 & -4 & -1 & -1 & -4 & -5 \\
\hline Gibraltar & Gibraltar & 2 & -6 & -1 & 0 & -4 & -2 \\
\hline Helsinki & Finland & -2 & -2 & -4 & -4 & -4 & -3 \\
\hline Jerusalem & Israel & -14 & -8 & -4 & -20 & 18 & -20 \\
\hline Kiev & Ukraine & -1 & -6 & -1 & -9 & -12 & -7 \\
\hline Lisbon & Portugal & -1 & -9 & -4 & 3 & -15 & -7 \\
\hline Ljubljana & Slovenia & 1 & -3 & 0 & -3 & -10 & -3 \\
\hline London & UK & -2 & -7 & -3 & 2 & -2 & -3 \\
\hline Luxemburg & Luxembourg & 2 & -3 & -1 & -6 & -8 & -6 \\
\hline Madrid & Spain & 0 & -9 & -4 & -6 & -6 & 2 \\
\hline Minsk & Belarus & -3 & -4 & -3 & -8 & -14 & -6 \\
\hline Monaco-Ville & Monaco & 7 & -9 & 2 & -1 & -12 & -4 \\
\hline Nicosia & Cyprus & -5 & -9 & -4 & 3 & -28 & -9 \\
\hline Oslo & Norway & -0 & -5 & -1 & -1 & -6 & -3 \\
\hline Paris & France & 3 & -2 & 1 & -4 & -6 & -5 \\
\hline Prague & Czech Republic & -2 & -3 & -3 & -2 & -6 & -3 \\
\hline Rabat & Morocco & -1 & -7 & 1 & 15 & 18 & 6 \\
\hline Riga & Latvia & -1 & -2 & -3 & -3 & -9 & -5 \\
\hline Rome & Italy & 1 & -5 & 4 & -1 & -11 & -6 \\
\hline Saint Helier & Jersey & -1 & -5 & -2 & 0 & -4 & -5 \\
\hline Saint Peter Port & Guernsey and A. & -2 & -6 & -2 & 1 & -4 & -4 \\
\hline San Marino & San Marino & -3 & -4 & 8 & -2 & -10 & -3 \\
\hline Sarajevo & Bosnia and $\mathrm{H}$. & -2 & -5 & -3 & -4 & -9 & -4 \\
\hline Skopje & Macedonia & 4 & -3 & 4 & 1 & -4 & -4 \\
\hline Sofia & Bulgaria & 1 & -2 & 2 & -5 & -8 & -4 \\
\hline Stockholm & Sweden & -1 & -5 & -2 & -3 & -7 & -7 \\
\hline Tallinn & Estonia & -3 & -2 & -4 & -7 & -10 & -8 \\
\hline Tirana & Albania & 2 & 1 & 2 & -1 & -10 & -5 \\
\hline Torshavn & Faroe Islands & 1 & -3 & -1 & -2 & -1 & -1 \\
\hline Tripoli & Libya & -8 & -4 & -3 & 9 & 9 & -8 \\
\hline Tunis & Tunisia & -5 & -7 & 0 & -11 & -12 & -5 \\
\hline Vaduz & Liechtenstein & -2 & -2 & -4 & -4 & -8 & -4 \\
\hline Valletta & Malta & -3 & -4 & 3 & 1 & -10 & 4 \\
\hline Vatican City & Vatican City & 1 & -5 & 4 & -1 & -11 & -6 \\
\hline Vienna & Austria & -3 & -7 & -2 & -5 & -8 & -2 \\
\hline Vilnius & Lithuania & -2 & -3 & -3 & -7 & -12 & -8 \\
\hline Warsaw & Poland & -3 & -2 & -2 & -2 & -10 & -6 \\
\hline Zagreb & Croatia & 2 & -2 & 3 & -4 & -9 & -4 \\
\hline
\end{tabular}




\section{References}

Christensen, O. B. and Kjellström, E.: Partitioning Uncertainty Components of Mean Climate and Climate Change in a Large Ensemble of European Regional Climate Model Projections, Climate Dynamics, https://doi.org/10.1007/s00382-020-05229-y, 2020.

Déqué, M., Rowell, D. P., Lüthi, D., Giorgi, F., Christensen, J. H., Rockel, B., Jacob, D., Kjellström, E., de Castro, M., and van den Hurk, B.: An Intercomparison of Regional Climate Simulations for Europe: Assessing Uncertainties in Model Projections, Climatic Change, 81, 53-70, https://doi.org/10.1007/s10584-006-9228-x, 2007.

Evin, G., Hingray, B., Blanchet, J., Eckert, N., Morin, S., and Verfaillie, D.: Partitioning Uncertainty Components of an Incomplete Ensemble of Climate Projections Using Data Augmentation, Journal of Climate, 32, 2423-2440, https://doi.org/10.1175/JCLI-D-18-0606.1, 2019.

Hawkins, E. and Sutton, R.: The Potential to Narrow Uncertainty in Regional Climate Predictions, Bulletin of the American Meteorological Society, 90, 1095-1107, https://doi.org/10.1175/2009BAMS2607.1, 2009.

Hingray, B. and Saïd, M.: Partitioning Internal Variability and Model Uncertainty Components in a Multimember Multimodel Ensemble of Climate Projections, Journal of Climate, 27, 6779-6798, https://doi.org/10.1175/JCLI-D-13-00629.1, 2014.

Northrop, P. J. and Chandler, R. E.: Quantifying Sources of Uncertainty in Projections of Future Climate, Journal of Climate, 27, 8793-8808, https://doi.org/10.1175/JCLI-D-14-00265.1, 2014.

Seneviratne, S. I., Nicholls, N., Easterling, D., Goodess, C. M., Kanae, S., Kossin, J., Luo, Y., Marengo, J., Mc Innes, K., Rahimi, M., Reichstein, M., Sorteberg, A., Vera, C., Zhang, X., Rusticucci, M., Semenov, V., Alexander, L. V., Allen, S., Benito, G., Cavazos, T., Clague, J., Conway, D., Della-Marta, P. M., Gerber, M., Gong, S., Goswami, B. N., Hemer, M., Huggel, C., Van den Hurk, B., Kharin, V. V., Kitoh, A., Klein Tank, A. M. G., Li, G., Mason, S., Mc Guire, W., Van Oldenborgh, G. J., Orlowsky, B., Smith, S., Thiaw, W., Velegrakis, A., Yiou, P., Zhang, T., Zhou, T., and Zwiers, F. W.: Changes in Climate Extremes and Their Impacts on the Natural Physical Environment, in: Managing the Risks of Extreme Events and Disasters to Advance Climate Change Adaptation, pp. 109-230, Cambridge University Press, https://doi.org/10.1017/CBO9781139177245.006, 2012.

Yip, S., Ferro, C. A. T., Stephenson, D. B., and Hawkins, E.: A Simple, Coherent Framework for Partitioning Uncertainty in Climate Predictions, Journal of Climate, 24, 4634-4643, https://doi.org/10.1175/2011JCLI4085.1, 2011. 